July 2004 • NREL/SR-510-36244

\title{
Biodiesel Production Technology
}

\section{August 2002-January 2004}

J. Van Gerpen, B. Shanks, and R. Pruszko lowa State University

D. Clements

Renewable Products Development Laboratory

\author{
G. Knothe \\ USDA/NCAUR
}
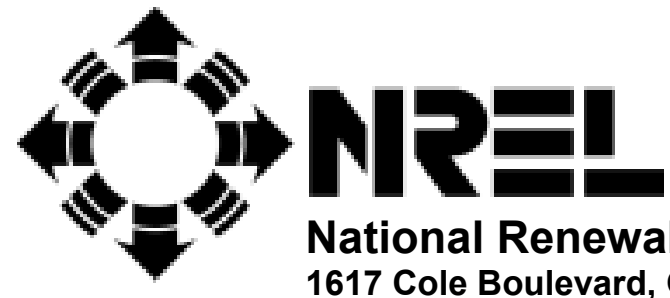

National Renewable Energy Laboratory 1617 Cole Boulevard, Golden, Colorado 80401-3393 303-275-3000 • www.nrel.gov

Operated for the U.S. Department of Energy Office of Energy Efficiency and Renewable Energy by Midwest Research Institute • Battelle 
July 2004 • NREL/SR-510-36244

\section{Biodiesel Production Technology}

\section{August 2002-January 2004}

J. Van Gerpen, B.Shanks, and R. Pruszko lowa State University

D. Clements

Renewable Products Development Laboratory

G. Knothe USDA/NCAUR

NREL Technical Monitor: K. Shaine Tyson

Prepared under Subcontract No. ACO-2-35016-01

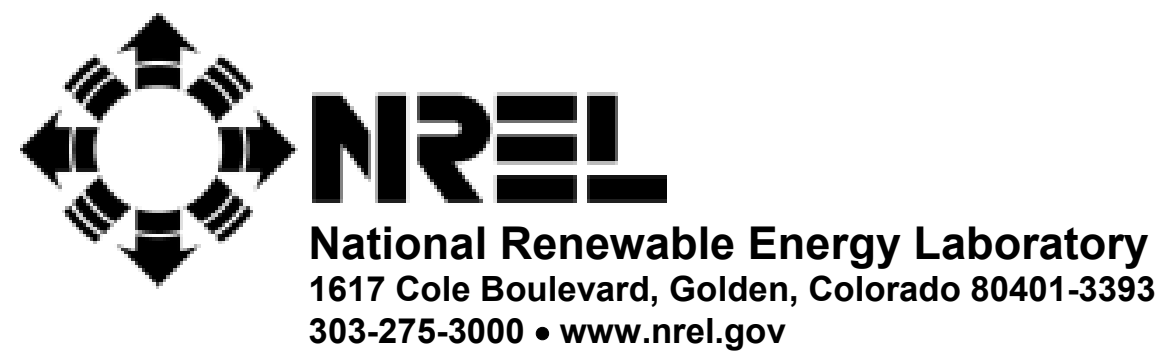

Operated for the U.S. Department of Energy Office of Energy Efficiency and Renewable Energy by Midwest Research Institute $\bullet$ Battelle Contract No. DE-AC36-99-G010337 


\section{NOTICE}

This report was prepared as an account of work sponsored by an agency of the United States government. Neither the United States government nor any agency thereof, nor any of their employees, makes any warranty, express or implied, or assumes any legal liability or responsibility for the accuracy, completeness, or usefulness of any information, apparatus, product, or process disclosed, or represents that its use would not infringe privately owned rights. Reference herein to any specific commercial product, process, or service by trade name, trademark, manufacturer, or otherwise does not necessarily constitute or imply its endorsement, recommendation, or favoring by the United States government or any agency thereof. The views and opinions of authors expressed herein do not necessarily state or reflect those of the United States government or any agency thereof.

Available electronically at http://www.osti.gov/bridge

Available for a processing fee to U.S. Department of Energy and its contractors, in paper, from:

U.S. Department of Energy

Office of Scientific and Technical Information

P.O. Box 62

Oak Ridge, TN 37831-0062

phone: 865.576.8401

fax: 865.576 .5728

email: mailto:reports@adonis.osti.gov

Available for sale to the public, in paper, from:

U.S. Department of Commerce

National Technical Information Service

5285 Port Royal Road

Springfield, VA 22161

phone: 800.553 .6847

fax: 703.605.6900

email: orders@ntis.fedworld.gov

online ordering: http://www.ntis.gov/ordering.htm

This publication received minimal editorial review at NREL 


\section{Biodiesel Production Technology}

\section{Background}

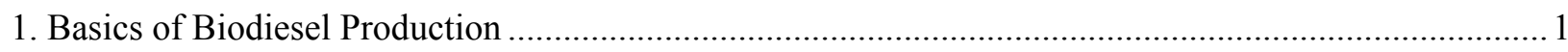

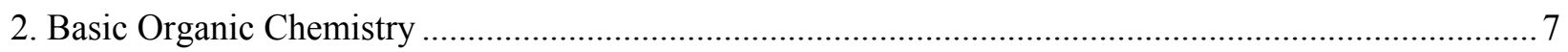

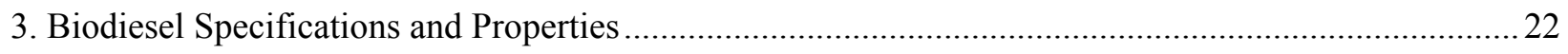

\section{Biodiesel Production Processes}

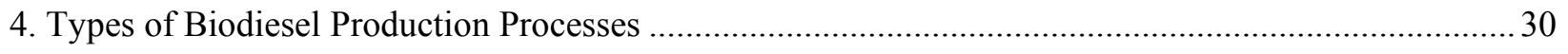

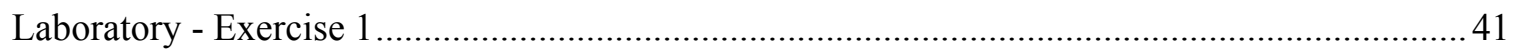

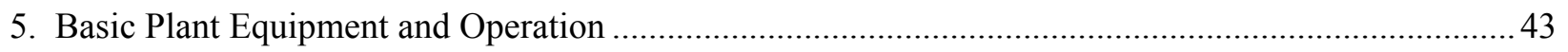

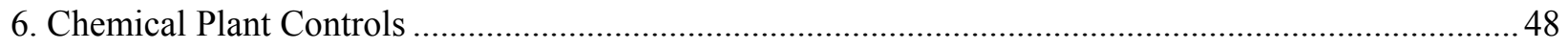

7. Pretreatment of High Free Fatty Acid Feedstocks ….................................................................. 52

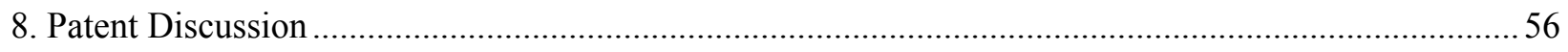

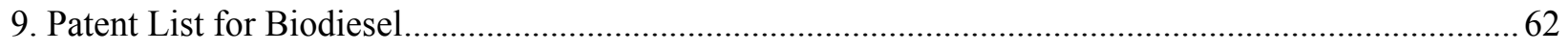

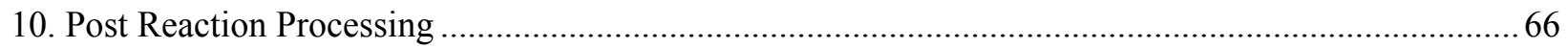

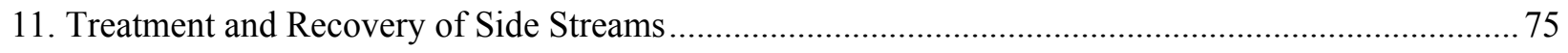

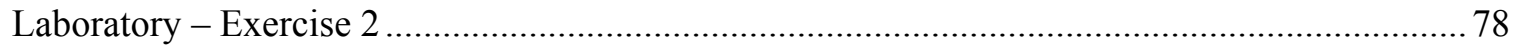

\section{Biodiesel Plant Logistics}

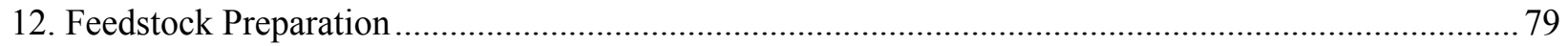

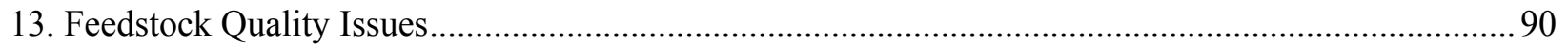

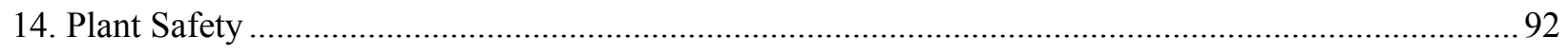

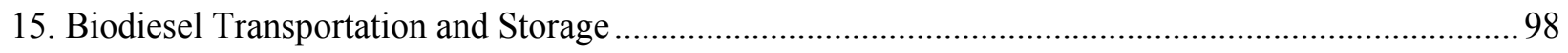

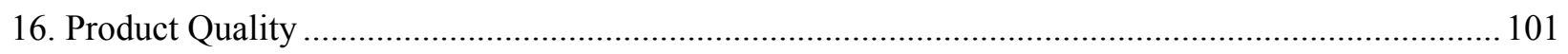

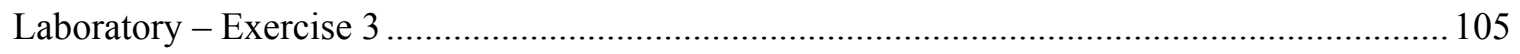




\section{Basics of Biodiesel Production}

Biodiesel is an alternative fuel for diesel engines that is gaining attention in the United States after reaching a considerable level of success in Europe. Its primary advantages are that it is one of the most renewable fuels currently available and it is also non-toxic and biodegradable. It can also be used directly in most diesel engines without requiring extensive engine modifications. The purpose of this book is to describe and explain the processes and issues involved in producing this new fuel.

The most cursory look at the literature relating to biodiesel will soon reveal the following relationship for prediction of biodiesel from fats and oils.

\section{$100 \mathrm{lbs}$ of oil $+10 \mathrm{lbs}$ of methanol $\rightarrow 100 \mathrm{lbs}$ of biodiesel $+10 \mathrm{lbs}$ of glycerol}

This equation is a simplified form of the following transesterfication reaction.

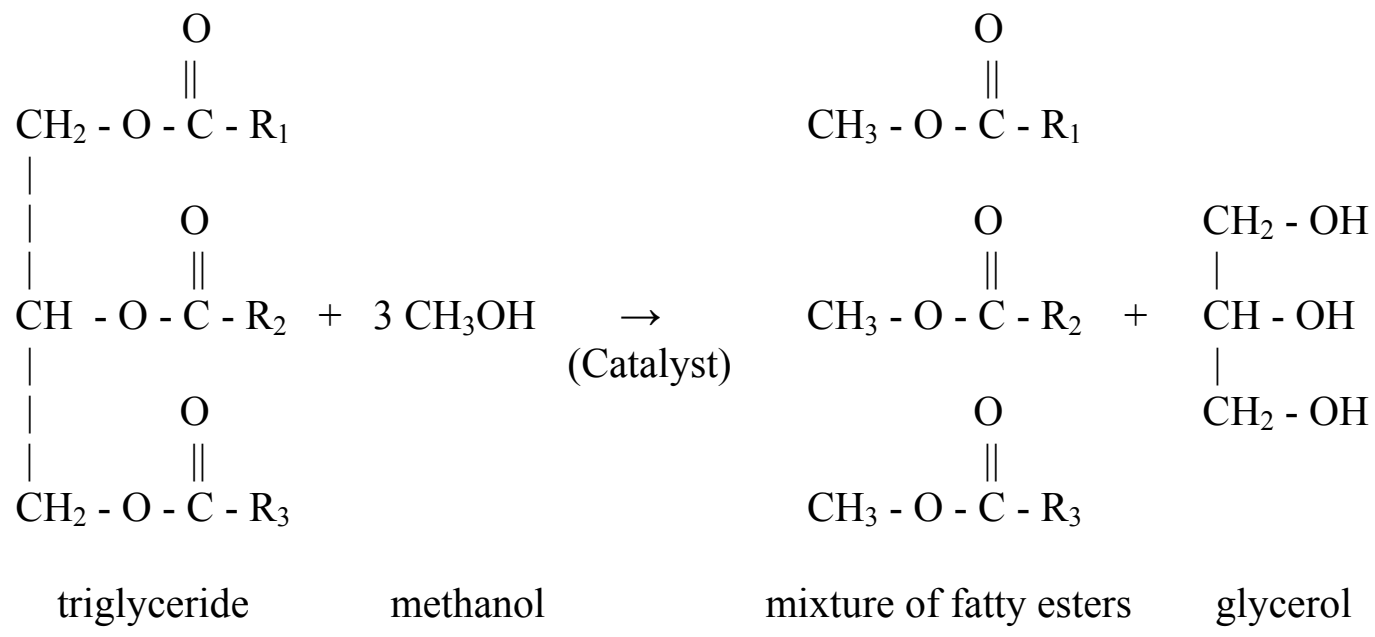

Figure 1. Transesterification Reaction

where $\mathrm{R}_{1}, \mathrm{R}_{2}$, and $\mathrm{R}_{3}$ are long chains of carbons and hydrogen atoms, sometimes called fatty acid chains. There are five types of chains that are common in soybean oil and animal fats (others are present in small amounts):

Palmitic: $\quad \mathrm{R}=-\left(\mathrm{CH}_{2}\right)_{14}-\mathrm{CH}_{3}$

Stearic: $\quad \mathrm{R}=-\left(\mathrm{CH}_{2}\right)_{16}-\mathrm{CH}_{3}$

Oleic:
16 carbons, (including the one that

$\mathrm{R}$ is attached to.) (16:0)

18 carbons, 0 double bonds (18:0) 
Table 1. Composition of Various Oils and Fats.

\begin{tabular}{|l|l|l|l|l|l|l|l|l|}
\hline Oil or fat & $14: 0$ & $16: 0$ & $18: 0$ & $18: 1$ & $18: 2$ & $18: 3$ & $20: 0$ & $22: 1$ \\
\hline Soybean & & $\mathbf{6 - 1 0}$ & $\mathbf{2 - 5}$ & $\mathbf{2 0 - 3 0}$ & $\mathbf{5 0 - 6 0}$ & $\mathbf{5 - 1 1}$ & & \\
\hline Corn & $\mathbf{1 - 2}$ & $\mathbf{8 - 1 2}$ & $\mathbf{2 - 5}$ & $\mathbf{1 9 - 4 9}$ & $\mathbf{3 4 - 6 2}$ & trace & & \\
\hline Peanut & & $\mathbf{8 - 9}$ & $\mathbf{2 - 3}$ & $\mathbf{5 0 - 6 5}$ & $\mathbf{2 0 - 3 0}$ & & & \\
\hline Olive & & $\mathbf{9 - 1 0}$ & $\mathbf{2 - 3}$ & $\mathbf{7 3 - 8 4}$ & $\mathbf{1 0 - 1 2}$ & trace & & \\
\hline Cottonseed & $\mathbf{0 - 2}$ & $\mathbf{2 0 - 2 5}$ & $\mathbf{1 - 2}$ & $\mathbf{2 3 - 3 5}$ & $\mathbf{4 0 - 5 0}$ & trace & & \\
\hline Hi linoleic Safflower & & $\mathbf{5 . 9}$ & $\mathbf{1 . 5}$ & $\mathbf{8 . 8}$ & $\mathbf{8 3 . 8}$ & & & \\
\hline Hi Oleic Safflower & & $\mathbf{4 . 8}$ & $\mathbf{1 . 4}$ & $\mathbf{7 4 . 1}$ & $\mathbf{1 9 . 7}$ & & & \\
\hline Hi Oleic Rapeseed & & $\mathbf{4 . 3}$ & $\mathbf{1 . 3}$ & $\mathbf{5 9 . 9}$ & $\mathbf{2 1 . 1}$ & $\mathbf{1 3 . 2}$ & & \\
\hline Hi Erucic Rapeseed & & $\mathbf{3 . 0}$ & $\mathbf{0 . 8}$ & $\mathbf{1 3 . 1}$ & $\mathbf{1 4 . 1}$ & $\mathbf{9 . 7}$ & $\mathbf{7 . 4}$ & $\mathbf{5 0 . 7}$ \\
\hline Butter & $\mathbf{7 - 1 0}$ & $\mathbf{2 4 - 2 6}$ & $\mathbf{1 0 - 1 3}$ & $\mathbf{2 8 - 3 1}$ & $\mathbf{1 - 2 . 5}$ & $\mathbf{. 2 - . 5}$ & & \\
\hline Lard & $\mathbf{1 - 2}$ & $\mathbf{2 8 - 3 0}$ & $\mathbf{1 2 - 1 8}$ & $\mathbf{4 0 - 5 0}$ & $\mathbf{7 - 1 3}$ & $\mathbf{0 - 1}$ & & \\
\hline Tallow & $\mathbf{3 - 6}$ & $\mathbf{2 4 - 3 2}$ & $\mathbf{2 0 - 2 5}$ & $\mathbf{3 7 - 4 3}$ & $\mathbf{2 - 3}$ & & & \\
\hline Linseed Oil & & $\mathbf{4 - 7}$ & $\mathbf{2 - 4}$ & $\mathbf{2 5 - 4 0}$ & $\mathbf{3 5 - 4 0}$ & $\mathbf{2 5 - 6 0}$ & & \\
\hline $\begin{array}{l}\text { Yellow grease } \\
\text { (Typical) }\end{array}$ & $\mathbf{2 . 4 3}$ & $\begin{array}{l}\mathbf{2 3 . 2 4} \\
\mathbf{1 6 : 1 3 . 7 9}\end{array}$ & $\mathbf{1 2 . 9 6}$ & $\mathbf{4 4 . 3 2}$ & $\mathbf{6 . 9 7}$ & $\mathbf{0 . 6 7}$ & & \\
\hline
\end{tabular}

Data derived from Organic Chemistry, W.W. Linstromberg, D.C. Heath and Co., Lexington, MA, 1970.

Linoleic: $\quad \mathrm{R}=-\left(\mathrm{CH}_{2}\right)_{7} \mathrm{CH}=\mathrm{CH}-\mathrm{CH}_{2}-\mathrm{CH}=\mathrm{CH}\left(\mathrm{CH}_{2}\right)_{4} \mathrm{CH}_{3}$

18 carbons, 2 double bonds (18:2)

Linolenic: $\quad \mathrm{R}=-\left(\mathrm{CH}_{2}\right)_{7} \mathrm{CH}=\mathrm{CH}-\mathrm{CH}_{2}-\mathrm{CH}=\mathrm{CH}-\mathrm{CH}_{2}-\mathrm{CH}=\mathrm{CH}-\mathrm{CH}_{2}-\mathrm{CH}_{3}$

18 carbons, 3 double bonds (18:3)

These chains are designated by two numbers separated by a colon. The first number designates the number of carbon atoms in the chain and the second number designates the number of double bonds. Note that the number of carbon atoms includes the carbon that is double bonded to the oxygen atom at one end of the fatty acid (called the carboxylic carbon). This is the end that the methanol attaches to when methyl esters are produced. Table 1 shows the percentages of each fatty acid chain present in common oils and fats.

For simplicity, consider an oil such as soybean oil to consist of pure triolein. Triolein is a triglyceride in which all three fatty acid chains are oleic acid. This is near the actual number of carbons and hydrogens and gives a molecular weight that is near the value for soybean oil. If triolein is reacted with methanol, the reaction will be that shown in Figure 2. Note that weights for each of the compounds in the reaction are given. These are based on the fact that one molecule of triolein reacts with 3 molecules of methanol to produce 3 molecules of methyl oleate, the biodiesel product, and one mole of glycerol. Chemists typically multiply all the terms of this equation by a large number that corresponds to the number of molecules in a quantity equal to the molecular weight of the substance. This quantity is called a mole of the substance. To calculate the molecular weight of triolein, we count the number of carbons in the molecule (57) and multiply 


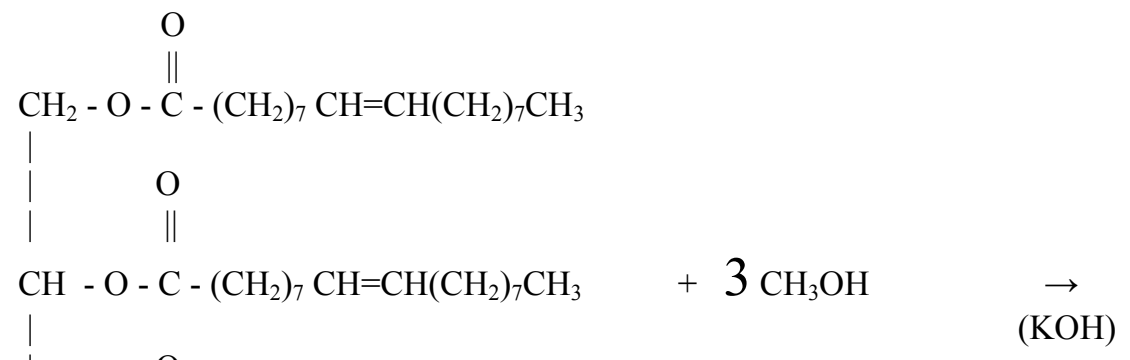

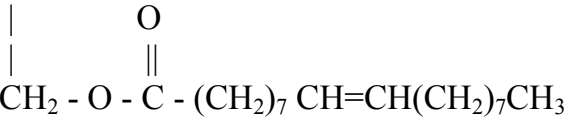

Triolein

$(\mathbf{8 8 5 . 4 6} \mathrm{g})$

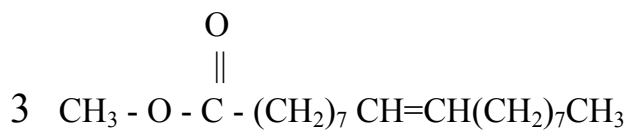

Methyl oleate (biodiesel)

$(3 \times 296.50=889.50 \mathrm{~g})$
Methanol

$(3 \times 32.04=96.12 \mathrm{~g})$

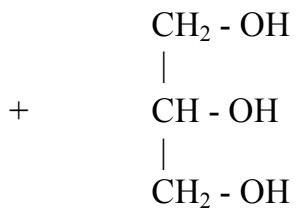

\section{Figure 2. Transesterification of Triolein}

this by 12.0111 , the molecular weight of carbon. Doing the same thing for hydrogen and oxygen gives:

$$
\begin{array}{cr}
57 \times 12.0111= & 684.63 \\
104 \times 1.00797= & 104.83 \\
\frac{6 \times 16.000=}{\text { Total }=} & \quad 896.00
\end{array}
$$

Therefore, the molecular weight of triolein is 885.46 and one mole of triolein weighs 885.46 grams. Three moles of methanol weigh $96.12 \mathrm{~g}, 3$ moles of methyl oleate weigh $889.50 \mathrm{~g}$, and 1 mole of glycerol weighs $92.10 \mathrm{~g}$.

We do not actually conduct the reaction this way. We usually add $60 \%$ to $100 \%$ excess methanol to ensure that the reaction goes to completion. In general, reactions can be encouraged to progress by adding an excess of one of the reactants or by removing one of the products. The reaction of triolein with $100 \%$ excess (XS) methanol is shown in Figure 3. 


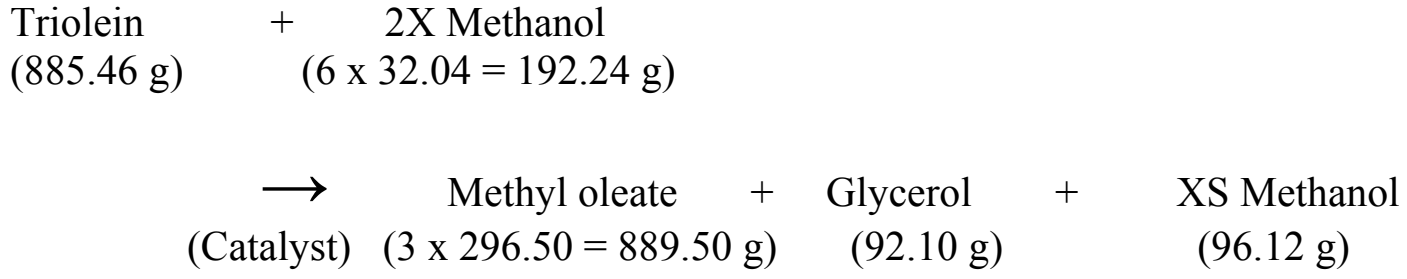

Figure 3. Transesterification of Triolein with $100 \%$ Excess Methanol

On the basis of $100 \mathrm{lb}$ of oil, the reaction mass balance with $100 \% \mathrm{XS}$ methanol becomes:

$100 \mathrm{lb}$ oil $+21.71 \mathrm{lb}$ methanol $\rightarrow 100.45 \mathrm{lb}$ biodiesel $+10.40 \mathrm{lb}$ glycerol $+10.86 \mathrm{lb}$ XS methanol

The reaction also requires about 1\% (based on the weight of oil) of sodium hydroxide or a similar catalyst that mostly ends up in the glycerol. These quantities can be converted to volumes by including the densities of the reactants and products given in Table 2.

Table 2. Densities of Biodiesel Reactants (kg/liter)

(from the Handbook of Chemistry and Physics, 51 ${ }^{\text {st }}$ Edition, CRC, 1970-1971.)

Triolein: $\quad 0.8988$

Methanol $\quad 0.7914$

Methyl Oleate $\quad 0.8739$

Glycerol $\quad 1.2613$

On a volume basis, the reaction becomes:

100 liters of oil +24.65 liters methanol

$\rightarrow 103.3$ liters methyl oleate +7.42 liters glycerol +12.33 liters XS methanol

\section{Product Quality}

The standard for biodiesel allows $0.24 \%$ total glycerol in the final product. What does this actually mean? It is clear that a molecule of a triglyceride can be considered to contain a molecule of glycerol, sometimes called the glycerol backbone. In the case of triolein, the mole of glycerol would weigh $92.10 \mathrm{~g}$ and the mole of triolein weighs $885.46 \mathrm{~g}$. Therefore, triolein can be considered to consist of $92.10 / 885.46=0.104$, or $10.4 \%$ glycerol. This glycerol is called bound glycerol because it is chemically bound to the triolein molecule. Bound glycerol can also be associated with monoglycerides and diglycerides, the partial reaction products of the conversion of triglycerides to alkyl esters. The structures of these molecules are shown in Figure 4. 


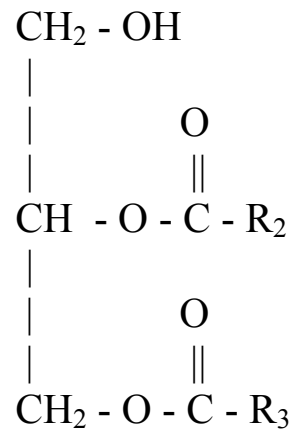

Diglyceride

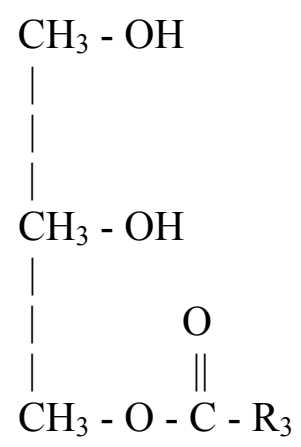

Monoglyceride

Figure 4. Chemical Structure of Diglyceride and Monoglyceride

Bound glycerol is added to any fully reacted glycerol, or free glycerol, that may still be in the biodiesel, to get the total glycerol. If the original oil contains $10.4 \%$ glycerol, and the final biodiesel can only contain a total glycerol level of $0.24 \%$, then the transesterification reaction must be

$$
\frac{10.4-0.24}{10.4} \times 100=97.7 \% \quad \text { or } 97.7 \% \text { complete }
$$

\section{Competing Reactions}

It is common for oils and fats to contain small amounts of water and free fatty acids. Free fatty acids consist of the long carbon chains described that are disconnected from the glycerol backbone. They are sometimes called carboxylic acids.<smiles>[R]C(=O)O</smiles>

Figure 5. Carboxylic Acid ( $R$ is a carbon chain)

The oleic group we have used earlier gives oleic acid, one of the free fatty acids that can be found in unrefined vegetable oils and animal fats.<smiles>CCCC=CCCC(=O)O</smiles>

Figure 6. Oleic Acid 
If an oil or fat containing a free fatty acid such as oleic acid is used to produce biodiesel, the alkali catalyst typically used to encourage the reaction will react with this acid to form soap. Figure 7 shows this reaction when the catalyst is potassium hydroxide $(\mathrm{KOH})$.

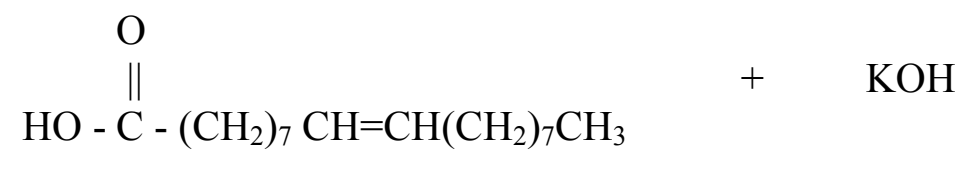

Oleic Acid

Potassium Hydroxide

$$
\begin{aligned}
& \stackrel{\mathrm{O}}{\|} \underset{\mathrm{K}^{+}{ }^{-} \mathrm{O}-\mathrm{C}-\left(\mathrm{CH}_{2}\right)_{7} \mathrm{CH}=\mathrm{CH}\left(\mathrm{CH}_{2}\right)_{7} \mathrm{CH}_{3}+\mathrm{H}_{2} \mathrm{O}}{\rightarrow} \\
& \text { Potassium oleate (soap) Water }
\end{aligned}
$$

Figure 7. Formation of Soap

This reaction is undesirable because it binds the catalyst into a form that does not contribute to accelerating the reaction. Excessive soap in the products can inhibit later processing of the biodiesel, including glycerol separation and water washing.

Water in the oil or fat can also be problem. When water is present, particularly at high temperatures, it can hydrolyze the triglycerides to diglycerides and form a free fatty acid. Figure 8 shows a typical hydrolysis reaction.

When an alkali catalyst is present, the free fatty acid will react to form soap following the reaction given earlier (Figure 7). When water is present in the reaction it generally manifests itself through excessive soap production. The soaps of saturated fatty acids tend to solidify at ambient temperatures so a reaction mixture with excessive soap may gel and form a semi-solid mass that is very difficult to recover.

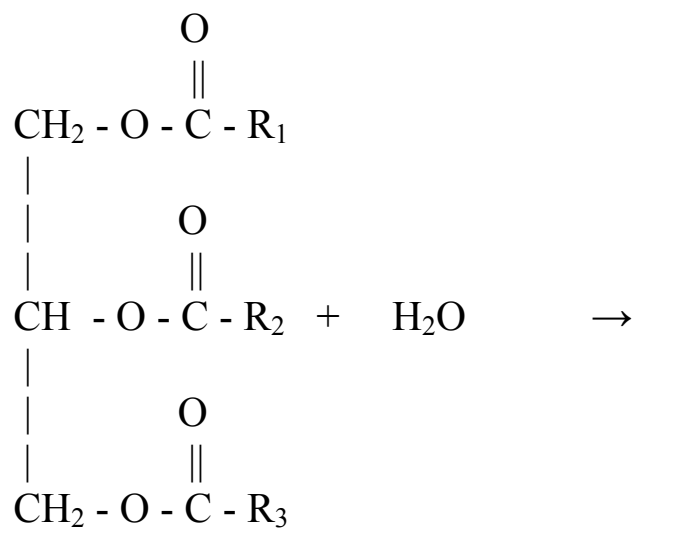

Triglyceride Water

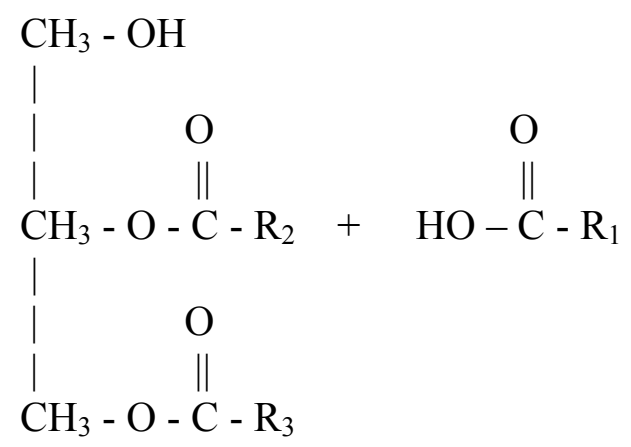

Diglyceride
Fatty acid

Figure 8. Hydrolysis of a Triglyceride to Form Free Fatty Acids 


\section{Basic Organic Chemistry}

As demonstrated in the preceding chapter, biodiesel production involves many chemical processes. In order to facilitate comprehension of the organic chemistry underlying biodiesel, its feedstocks, production and analysis, we will introduce some basic chemistry definitions. Some or parts of some definitions given here are taken from Webster's Third New International Dictionary, published by Merriam-Webster, Springfield, MA, 1993.

\section{Basic Chemistry}

Chemistry is the science that deals with the composition, structure, and properties of substances and of the transformations that these substances undergo. In most cases, those transformations are called reactions.

More than 100 elements are now known and most of them occur naturally. An element is a substance that cannot be separated into simpler substances by ordinary chemical means. Elements are arranged in a systematic fashion based on their atomic numbers (see definition below) and properties in the periodic table.

An atom is the smallest particle of an element that can exist either alone or in combination with similar particles (other atoms) of the same or of a different element. It is the smallest particle of an element that enters into the composition of molecules (which leads us to the definition of a molecule below). An element is comprised exclusively of atoms having the same atomic number. The atoms of each element have a specific atomic weight, which is the average relative weight of an element as it occurs in nature referred to some element taken as standard. Atoms are composed of protons, electrons and neutrons, which are responsible for the atomic weight. The nucleus of an atom consists of the protons and neutrons. The nucleus is the bulk of the weight of the atom. Electrons are smaller and lighter and they orbit the nucleus. It is very important that a proton has a positive charge and an electron has a negative charge. The number of protons, neutrons and electrons that comprise an atom give the atom its properties and ultimately the properties of the element. Thus, the element with one proton (and one electron) is hydrogen. "Adding" another proton (and electron as well as neutrons) gives the next element (helium), etc. Carbon has six protons and six electrons while oxygen has eight protons and electrons.

A molecule is the smallest particle of an aggregate of at least two connected atoms of either the same or different elements and has a combination of properties (chemical and physical) that is specific to it. Each molecule has a molecular weight, which is the weight of the molecule calculated as the sum of the atomic weights of its constituent atoms.

A compound is a chemically distinct substance formed by the union of two or more ingredients (as elements) in definite proportion by weight and with definite structural arrangement.

A mole is the quantity of a chemical substance that has a weight in mass units (in grams) numerically equal to the molecular weight or that in the case of a gas has a volume occupied by such a weight under specified conditions (as 22.4 liters at $0^{\circ} \mathrm{C}$ and a pressure of 760 millimeters 
of mercury). Thus, the number of molecules $\left(6.022 \times 10^{23}\right)$ in one mole of any given substance is equal to the number of molecules in one mole on any other substance. The concept of mole is very important in chemistry for calculating the amounts of compounds that undergo a reaction with each other. Often reactions are influenced by the amounts of compounds reacted with each other, the molar ratio (a related term to molar ratio is stoichiometry of a reaction).

Elements are in most cases comprised of the same kind of elementary particles known as atoms. Atoms can form aggregates known as molecules. Molecules can consist of atoms of many different elements or of atoms of one element. If a molecule is comprised of atoms of different elements, it is also known as a compound.

Each element has a symbol, for example $\mathrm{H}$ for hydrogen (hydrogen is the lightest element, each atom consisting only of one proton and one electron, see above; a hydrogen atom with the electron removed is referred to as a proton that can be written as $\mathrm{H}^{+}$) and $\mathrm{O}$ for oxygen, so that a chemical formula for each compound can be written. If more than one atom of an element is in the compound, a subscript number indicates that. Thus, water, as a compound comprised of two hydrogen atoms and one oxygen atom, has the molecular formula $\mathrm{H}_{2} \mathrm{O}$. In other words, each water molecule is composed of two hydrogen atoms and one oxygen atom.

\section{Organic chemistry}

Although there are many compounds that bridge the gap, most compounds are still classified either as inorganic or organic. The reason goes back far into the history of chemistry when it was assumed that all compounds came either from inanimate matter (inorganic compounds) or living matter (organic compounds). With some exceptions (an example is carbon dioxide, $\mathrm{CO}_{2}$ ) that will not concern us here, organic chemistry is the chemistry of the element carbon (symbol C). If organic chemistry deals only with the chemistry of one element, then why is it distinguished from inorganic chemistry? The reason is that most known compounds (approximately 90\%) are organic compounds. If you think of the astounding variety of plant and animal life and that, on a basic level, organic compounds are responsible for that life, it becomes clear why there are so many organic compounds. More scientifically, carbon atoms are very versatile in forming compounds with atoms of other elements. So why are we going to deal with some organic chemistry here? The answer is that both biodiesel and conventional diesel fuel (derived from petroleum) are mixtures of organic compounds.

Besides carbon, several other elements are very important to organic chemistry. Three especially important elements are hydrogen, oxygen, and nitrogen. Other important elements are phosphorus, sulfur and the so-called halogens (fluorine, chlorine, bromine and iodine). The following table contains some basic information on common elements (except halogens) in organic chemistry. 
Table 3. Some elements of importance to organic chemistry.

\begin{tabular}{|l|l|l|l|}
\hline Name & Symbol & Atomic Number & Atomic Weight \\
\hline Carbon & $\mathrm{C}$ & 6 & 12.011 \\
\hline Hydrogen & $\mathrm{H}$ & 1 & 1.008 \\
\hline Nitrogen & $\mathrm{N}$ & 7 & 14.007 \\
\hline Oxygen & $\mathrm{O}$ & 8 & 15.9994 \\
\hline Phosphorus & $\mathrm{P}$ & 15 & 30.974 \\
\hline Sulfur & $\mathrm{S}$ & 16 & 32.06 \\
\hline
\end{tabular}

For biodiesel purposes, carbon, hydrogen and oxygen are especially important and it is a good idea to memorize their atomic weights. For (rough) estimates, it suffices to just use the values $\mathrm{H}$ $=1, \mathrm{C}=12, \mathrm{O}=16$, etc.

When atoms form molecules, they connect via bonds. For organic chemistry, it is extremely important that each carbon atom in a molecule forms four bonds to other atoms. These other atoms can be other carbon, hydrogen, or oxygen or atoms of other elements. The bonds can be formed to a mixture of other atoms, for example, one carbon atom can form a bond to one hydrogen, one oxygen and two carbon atoms to meet the requirement of four bonds. When a carbon atom bonds to four other atoms, regardless of the nature of these atoms, these bonds are single bonds. Hydrogen can form only one bond to other atoms and oxygen can form two bonds to other atoms. It is also extremely important that carbon atoms can form double bonds or triple bonds. Compounds that have double or triple bonds are often called unsaturated compounds (can you explain why?). Thus, one carbon atom can be connected to an oxygen atom by a single bond, another carbon by a single bond, and another carbon by a double bond to meet the requirement of four bonds. Two carbon atoms can also be connected by triple bonds. A carbon atom and an oxygen atom can also be connected by a double bond (obviously, then the oxygen atom cannot form a bond with another atom). This versatility in bonding is responsible for the many different, often complex, structures of organic compounds.

As in the case of water discussed above, organic molecules can be written as formulas, for example, $\mathrm{CH}_{4}$ for methane. For more complex molecules, it is a good idea and indeed customary to write structural formulas because of the many possible structures for a given combination of carbon atoms with other atoms (a feature known as isomerism). That way, features like double or triple bonds can also be indicated by the corresponding number of lines between carbon atoms or carbon and other atoms. Examples of some simple organic molecules containing only carbon, hydrogen, and oxygen that also show the diversity of organic compounds (check to see that there are always four bonds per carbon) are shown in Figure 9.

Organic compounds can be categorized into various classes of compounds. In the above examples, note that methanol, ethanol (the alcohol contained in alcoholic beverages), and 1- and 2-propanol (note the difference in 1- and 2-propanol; what does the difference in nomenclature indicate?) belong to the class of compounds known as alcohols. Their common feature is the 


$\begin{array}{cccccc} & & & \mathrm{O} \\ \mathrm{CH}_{4} & \mathrm{CH}_{3}-\mathrm{CH}_{3} & \mathrm{CH}_{3}-\mathrm{CH}_{2}-\mathrm{CH}_{3} & \mathrm{CH}_{2}=\mathrm{CH}_{2} & \mathrm{CH} \equiv \mathrm{CH} & \mathrm{CH}_{3}-\mathrm{C}-\mathrm{CH}_{3} \\ \text { Methane } & \text { Ethane } & \text { Propane } & \begin{array}{c}\text { Ethylene } \\ \text { (Ethene) }\end{array} & \begin{array}{c}\text { Acetylene } \\ \text { (Ethyne) }\end{array} & \begin{array}{c}\text { Acetone } \\ \text { (Propanone) }\end{array}\end{array}$

$\mathrm{CH}_{3} \mathrm{OH}$

Methanol<smiles>OCC(O)CO</smiles>

Glycerol

(Glycerine; 1,2,3-Propanetriol)

Figure 9. Simple Organic Molecules

presence of an $\mathrm{OH}$ group (also known as hydroxy group). A feature such as an $\mathrm{OH}$ group is also known as a functional group. Besides $\mathrm{OH}$, there are many more functional groups. A functional group usually imparts specific properties and the propensity to undergo certain reactions to a molecule. There are innumerable compounds that belong to the class of compounds known as alcohols. Many organic molecules contain the characteristics of several or even many classes of compounds usually because they contain several or many different functional groups.

Note how the names of alcohols are derived from the compounds in the top row known as hydrocarbons (hydrocarbons are compounds containing carbon and hydrogen) by dropping the -e and adding the suffix -ol (Why is the name "glycerol" preferable to "glycerine" for the same compound?). Similarly, note that the $\mathrm{CH}_{3}$ moiety as it is found in methyl esters is called a methyl group and $\mathrm{CH}_{2} \mathrm{CH}_{3}$ is called ethyl, again a systematic change in nomenclature. Such changes in nomenclature, especially rational nomenclature, are common in organic chemistry. For some of the compounds, more than one name is given. This results of many compounds having so-called trivial names besides their rational names (historically, the trivial names are older than the rational names). Trivial names are important because for many organic compounds of more complex structure the rational names become unwieldy, so that the trivial names are much easier to learn and use. 

$\mathrm{R}-\mathrm{OH}$
$\mathrm{O}$
R-C-OH
$\mathrm{O}$
$\mathrm{R}_{1}-\mathrm{C}-\mathrm{O}-\mathrm{R}_{2}$
$\mathrm{O}$
$\mathrm{R}_{1}-\mathrm{C}-\mathrm{R}_{2}$
$\underset{\text { R-C-H }}{\text { O }}$
Alcohol Carboxylic acid
Ester
Ketone
Aldehyde

Figure 10. Classes of Organic Molecules

When reading (or writing) the structural formulas of organic compounds, you will often encounter $\mathrm{R}$ or $\mathrm{R}_{1}, \mathrm{R}_{2}$ or $\mathrm{R}_{3}$, etc. " $\mathrm{R}$ " means "organic rest" and is an abbreviation for a part of the molecule which then has to be defined. General formulas for some classes of compounds can be written as shown in Figure 10.

The specific structural features of organic compounds (such as the hydroxy groups in alcohols) are responsible for the fact that many compounds can react with other compounds to form new compounds. The new may then belong to a different class of compounds. These structural features are also responsible for the differences in physical properties (melting point, boiling point, etc.) of the organic compounds. For example, acetic acid can react with methanol to form methyl acetate (which belongs to the ester class of compounds; esters are formed by the reaction of an acid with an alcohol), in which case water is also formed as a byproduct:

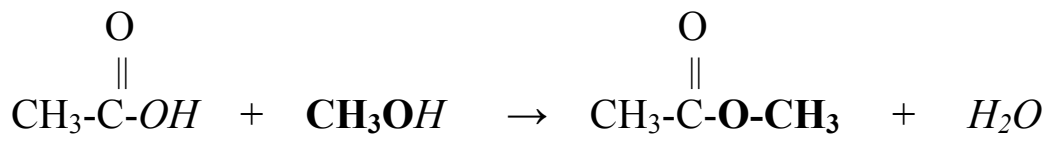

Figure 11. Chemical Reaction to Form Methyl Acetate

You can also perform the back reaction in which methyl acetate reacts with water to form acetic acid and methanol. When methanol is the alcohol participating in the reaction, the ester formed is a methyl ester, when ethanol is the alcohol participating in the reaction, an ethyl ester is formed.

\section{Vegetable oils and biodiesel}

Now we can start to deal with biodiesel. As you know, biodiesel is derived from vegetable oils. The major components of vegetable oils are triglycerides. The term triacylglycerols is being used more and more, but we will use the classical term in this discussion. Triglycerides are esters of glycerol (see above; an alcohol with a hydroxy group on each of its three carbon atoms) with long-chain acids, commonly called fatty acids. Tables 4 and 5 list the most common fatty acids and their corresponding methyl esters.

Note from the comparison of the rational names of the fatty acids with their structural formulas how the position of the double bonds is defined by numbers. The number of carbon atoms is counted by beginning with the first carbon having the functional group defining the fatty compound as acid or ester. As you can see from the former example (for example, 1-propanol and 2-propanol), this way of counting holds for other functional groups as well. The trivial names of fatty acids and their esters are far more commonly used than their rational names. 
Table 4. Chemical Structure of Common Fatty Acids and Their Methyl Esters

\begin{tabular}{|c|c|c|c|}
\hline $\begin{array}{l}\text { Fatty acid (trivial name / } \\
\text { rational name); }\end{array}$ & Structure ${ }^{\text {a) }}$ & $\begin{array}{l}\text { Common } \\
\text { acronym } \\
\end{array}$ & $\begin{array}{l}\text { Methyl ester (trivial } \\
\text { name / rational name) }\end{array}$ \\
\hline $\begin{array}{l}\text { Palmitic acid / } \\
\text { Hexadecanoic acid; }\end{array}$ & $\mathrm{R}-\left(\mathrm{CH}_{2}\right)_{14}-\mathrm{CH}_{3}$ & $\mathrm{C} 16: 0$ & $\begin{array}{l}\text { Methyl palmitate / } \\
\text { Methyl hexadecanoate }\end{array}$ \\
\hline $\begin{array}{l}\text { Stearic acid / Octadecanoic } \\
\text { acid }\end{array}$ & $\mathrm{R}-\left(\mathrm{CH}_{2}\right)_{16}-\mathrm{CH}_{3}$ & C18:0 & $\begin{array}{l}\text { Methyl stearate / } \\
\text { Methyl octadecanoate }\end{array}$ \\
\hline $\begin{array}{l}\text { Oleic acid / } 9(Z)- \\
\text { octadecenoic acid }\end{array}$ & $\begin{array}{l}\mathrm{R}-\left(\mathrm{CH}_{2}\right)_{7}-\mathrm{CH}=\mathrm{CH}-\left(\mathrm{CH}_{2}\right)_{7^{-}} \\
\mathrm{CH}_{3}\end{array}$ & C18:1 & $\begin{array}{l}\text { Methyl oleate / Methyl } \\
\text { 9(Z)-octadecenoate }\end{array}$ \\
\hline $\begin{array}{l}\text { Linoleic acid / } 9(Z), 12(Z)- \\
\text { octadecadienoic acid; }\end{array}$ & $\begin{array}{l}\mathrm{R}-\left(\mathrm{CH}_{2}\right)_{7}-\mathrm{CH}=\mathrm{CH}-\mathrm{CH}_{2-} \\
\mathrm{CH}=\mathrm{CH}-\left(\mathrm{CH}_{2}\right)_{4}-\mathrm{CH}_{3}\end{array}$ & C18:2 & $\begin{array}{l}\text { Methyl linoleate / } \\
\text { Methyl 9(Z), 12(Z)- } \\
\text { octadecadienoate }\end{array}$ \\
\hline $\begin{array}{l}\text { Linolenic acid / } \\
9(Z), 12(Z), 15(Z)- \\
\text { octadecatrienoic acid; }\end{array}$ & $\begin{array}{l}\mathrm{R}-\left(\mathrm{CH}_{2}\right)_{7}-\left(\mathrm{CH}=\mathrm{CH}-\mathrm{CH}_{2}\right)_{3}- \\
\mathrm{CH}_{3}\end{array}$ & C18:3 & $\begin{array}{l}\text { Methyl linolenate / } \\
\text { Methyl } \\
9(Z), 12(Z), 15(Z)- \\
\text { octadecadienoate }\end{array}$ \\
\hline
\end{tabular}

a) $\mathrm{R}=\mathrm{COOH}\left(\mathrm{CO}_{2} \mathrm{H}\right)$ or $\mathrm{COOCH}_{3}\left(\mathrm{CO}_{2} \mathrm{CH}_{3}\right) ;\left(\mathrm{CH}_{2}\right)_{7}=\mathrm{CH}_{2}-\mathrm{CH}_{2}-\mathrm{CH}_{2}-\mathrm{CH}_{2}-\mathrm{CH}_{2}-\mathrm{CH}_{2}-\mathrm{CH}_{2}$, etc.

Table 5. Characteristics of Common Fatty Acids and Their Methyl Esters

\begin{tabular}{|l|l|l|l|}
\hline $\begin{array}{l}\text { Fatty acid } \\
\text { Methyl ester }\end{array}$ & Formula & Molecular weight & $\begin{array}{l}\text { Melting point } \\
\left({ }^{\circ} \mathbf{C}\right)\end{array}$ \\
\hline Palmitic acid & $\mathrm{C}_{16} \mathrm{H}_{32} \mathrm{O}_{2}$ & 256.428 & $63-64$ \\
Methyl palmitate & $\mathrm{C}_{17} \mathrm{H}_{34} \mathrm{O}_{2}$ & 270.457 & 30.5 \\
\hline Stearic acid & $\mathrm{C}_{18} \mathrm{H}_{36} \mathrm{O}_{2}$ & 284.481 & 70 \\
Methyl stearate & $\mathrm{C}_{19} \mathrm{H}_{38} \mathrm{O}_{2}$ & 298.511 & 39 \\
\hline Oleic acid & $\mathrm{C}_{18} \mathrm{H}_{34} \mathrm{O}_{2}$ & 282.465 & 16 \\
Methyl oleate & $\mathrm{C}_{19} \mathrm{H}_{36} \mathrm{O}_{2}$ & 296.495 & -20 \\
\hline Linoleic acid & $\mathrm{C}_{18} \mathrm{H}_{32} \mathrm{O}_{2}$ & 280.450 & -5 \\
Methyl linoleate & $\mathrm{C}_{19} \mathrm{H}_{34} \mathrm{O}_{2}$ & 294.479 & -35 \\
\hline Linolenic acid & $\mathrm{C}_{18} \mathrm{H}_{30} \mathrm{O}_{2}$ & 278.434 & -11 \\
Methyl linolenate & $\mathrm{C}_{19} \mathrm{H}_{32} \mathrm{O}_{2}$ & 292.463 & $-52 /-57$ \\
\hline
\end{tabular}

A glance at the differences in the nomenclature will indicate why. Often fatty acids and their esters are referred to, especially in writing, by the abbreviations given in the last column of Table 4. (Question: How do those abbreviations arise?)

All double bonds in the rational nomenclature of fatty acids and esters are also defined by a letter $(Z)$, which is the result of the possibility of two kinds of double bonds. These two kinds of double bonds are a result of how the other parts of the molecule are attached in space to the carbon atoms forming the double bond. Besides this technical aspect, we will not discuss any 
further details of such arrangements of molecules in space except nomenclature. In rational nomenclature, the counterpart to $Z$ is defined by the letter $E$. However, in the literature the terms cis and trans are also often used. In organic chemistry, a cis double usually, but not always, corresponds to a $Z$ double bond and a trans double bond usually, but not always, corresponds to an $E$ double bond. In the case of the fatty acids we are interested in here, cis and $Z$ correspond to each other as do trans and $E$. However, each of these fatty acids has a counterpart in which the double bonds are trans (or $E$ ), but their occurrence (in nature) is far less common. (Question: Have you heard health reports on trans fatty acids?) Under certain conditions, the double bonds can change from $Z$ to $E$. These conditions include heating and types of reactions called hydrogenations (a hydrogenation reaction is the production of more saturated fats from unsaturated ones by adding hydrogen to double bonds).

The two kinds of double bonds cause the compounds to have different physical properties such as melting point. For example, while the melting point of oleic acid is $15^{\circ} \mathrm{C}$ (compare to that of methyl oleate above; note that fatty acids have higher melting points than their corresponding methyl esters), that of elaidic acid (the acid in which the $Z$ double bond in oleic acid is replaced by $E$; the methyl ester would be called methyl elaidate) is $45^{\circ} \mathrm{C}$. The existence of different compounds for the same formula (both oleic acid and elaidic acid are $\mathrm{C}_{18} \mathrm{H}_{34} \mathrm{O}_{2}$; their methyl esters are $\mathrm{C}_{19} \mathrm{H}_{36} \mathrm{O}_{2}$ ) is called isomerism. The corresponding compounds are then isomers. There are many different kinds of isomerism. A more detailed discussion of isomerism is beyond the scope of this course.

It is extremely important to realize that vegetable oils are mixtures of triglycerides from various fatty acids. The composition of vegetable oils varies with the plant source. Often the terms fatty acid profile or fatty acid composition are used to describe the specific nature of fatty acids occurring in fats and oils. Several examples were described in Chapter 1 and profiles for common vegetable oils and animal fats were given in Table 1 . The chemical and physical properties of fats and oils and the esters derived from them vary with the fatty acid profile. For example, imagine how mixtures containing larger amounts of methyl palmitate or methyl stearate will solidify at higher temperatures than those containing lesser amounts (see melting points in Table 5).

The corresponding esters of those fatty acids in Tables 4 and 5 with glycerol, are known as tripalmitin, tristearin, triolein, trilinolein and trilinolenin. (Exercise: Calculate the molecular weights of these triglycerides)

In a variation of the formation of esters from acids and alcohols as discussed above, an ester can react with another alcohol. In that case, the new alcohol is derived from the original ester is formed and the new ester is derived from the original alcohol. Thus, an ethyl ester can react with methanol to form a methyl ester and ethanol. This process is called transesterification.

Transesterification is extremely important for biodiesel. Biodiesel as it is defined today is obtained by transesterifying the triglycerides with methanol. Methanol is the preferred alcohol for obtaining biodiesel because it is the cheapest (and most available) alcohol. However, for the reaction to occur in a reasonable time, a substance called a catalyst (catalysts are substances that, often present in small amounts, accelerate the speed of a reaction; in many cases virtually no reaction would occur without a catalyst), must be added to the mixture of the vegetable oil and 
methanol. The transesterification reaction for biodiesel production was provided in Figure 1 and is repeated in Figure 12 in more generic form.

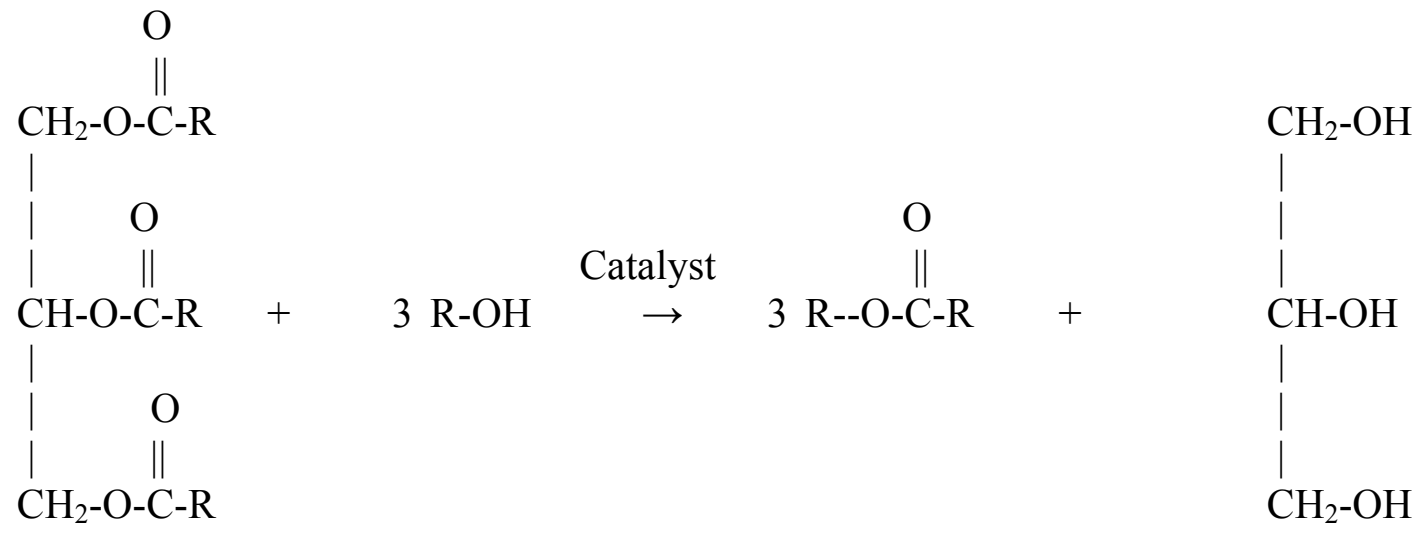

Triacylglycerol

(Vegetable oil)
Alcohol

Alkyl ester

(Biodiesel)
Glycerol

Figure 12. The transesterification reaction.

$\mathrm{R}$ is a mixture of various fatty acid chains. The alcohol used for producing biodiesel is usually methanol (where the R- corresponds to $\mathrm{CH}_{3}$ ).

As noted in the figure caption, $\mathrm{R}$ can be any of the fatty acid chains (for example, $-\left(\mathrm{CH}_{2}\right)_{14}-\mathrm{CH}_{3}$; see Table 4 for more examples; note how R or anything containing it is flexible and must be defined each time it is used). The number 3 indicates the number of moles needed to satisfy the formation of the methyl esters. When one mole is necessary, the number 1 is usually not written. It is also important to keep it mind that this only formally states the molar ratios of starting materials and products. In practice, the molar ratios may need to vary to obtain a nearly complete reaction.

For the transesterification to occur, usually 6 moles of alcohol are used for every mole of triglyceride, which is more than the equation indicates. The reason is that the reaction is desired to proceed in the direction of the arrow, i.e., to the right. In other terms, the equilibrium of the reaction needs to be shifted toward the right side of the equation. As the term "equilibrium" indicates, not all reactions easily proceed to completion and after some time the starting materials and reaction products are present in constant amounts (the equilibrium has been attained). In many cases, the fact that a reaction can proceed in the reverse fashion (from right to left in the equation) also plays a role in formation of the equilibrium. To force the equilibrium in the direction of the products (as is almost always desired), one or more parameter(s) of the reaction may need to be changed. Such parameters include the molar ratio as well as others such as temperature, pressure and use of a catalyst.

In accordance with the names of fatty acids and their esters (see Tables 4 and 5), the methyl ester of soybean oil is often called methyl soyate. The term soybean oil methyl ester (SME) is 
also very common. The same holds for the esters of other vegetable oils. Another common abbreviation that is commonly used is FAME (fatty acid methyl ester).

Besides triglycerides, mono- and diglycerides can also exist. They are formed as intermediates during the transesterification reaction. This is one of the problems when conducting chemical reactions in general, not only the transesterification reaction. It is almost always the goal of chemical reactions to obtain products that are as pure as possible. However, hardly any chemical reaction proceeds to full completion (see the discussion of equilibrium above). Therefore, often intermediates (in the case of transesterification the intermediates are partially reacted triglycerides, i.e., the mono- and diglycerides) can contaminate the final product. Other materials that can contaminate biodiesel are residual methanol (or other alcohol), glycerol, and catalyst.

When the transesterification reaction is conducted, you can visually observe that not all materials can be readily mixed with each other. A term often used for this phenomenon is that there are two phases. At the start of the reaction, you can notice that methanol and vegetable oil do not readily mix. At the end of the reaction, you can notice that there are two layers (phases), one consisting mainly of glycerol, the other of the methyl esters. Obviously, glycerol and methyl esters do not mix readily. How readily one compound will dissolve in another depends on the structural features of the compounds, for example the existence of $\mathrm{OH}$ groups. Thus, compounds containing $\mathrm{OH}$ groups and those not containing $\mathrm{OH}$ groups often will not readily mix.

(Question: Why is water effective in "washing" biodiesel after the transesterification reaction?)

The catalyst used for carrying out the transesterification is usually sodium hydroxide $(\mathrm{NaOH})$ or potassium hydroxide $(\mathrm{KOH})$. These compounds belong to a class of materials known as bases and also are inorganic compounds (inorganic compounds are often used in organic chemistry for carrying out or catalyzing reactions). Other bases are also suitable for the transesterification reaction. The counterparts of bases are known as acids. Many acids can also be used as catalysts in the transesterification reaction. However, the base-catalyzed reaction has advantages such as a higher reaction rate. More details are covered in the production part of this book.

In the soap formation reaction described in Figure 7, a fatty acid and base reacted to form a new compound, which was called soap, and water. Compounds such as soap, in which the hydrogen (proton) of an acid has been replaced with a metal ion, are often called salts. The reason that such compounds exist is that materials such as $\mathrm{NaOH}$ (or $\mathrm{KOH}$ ) can split apart (dissociate) in a fashion that gives $\mathrm{Na}^{+}$and $\mathrm{OH}^{-}\left(\right.$or $\mathrm{K}^{+}$and $\mathrm{OH}^{-}$) in which the protons and electrons are not evenly distributed, leading to charged particles. Thus, having the same charge, $\mathrm{Na}^{+}$or $\mathrm{K}^{+}$can replace $\mathrm{H}^{+}$here.

Another important aspect of the chemistry of fatty acids and their esters is that the more unsaturated fatty acids and their esters (linoleic, linolenic) can relatively easily react with air (more specifically, the oxygen in air) and form degradation products with time (the time depends also on other factors such as temperature). This is due to the existence of $\mathrm{CH}_{2}$ between two carbons double-bonded to other carbons (see the structural formulas in Table 4). 


\section{Why are methyl esters of fatty acids suitable as diesel fuel?}

Earlier, we briefly discussed compounds such as methane, ethane, and propane. These compounds belong to a class of compounds called alkanes. Alkanes are compounds consisting only of carbon and hydrogen and they only contain single bonds. Therefore, the general formula for straight-chain alkanes is $\mathrm{CH}_{3}-\left(\mathrm{CH}_{2}\right)_{\mathrm{n}}-\mathrm{CH}_{3}$. Other alkanes, for example with branching in the chain, or with the carbons arranged in a ring, can also exist. However, in each case, the carbons are connected only with single bonds.

When dealing with diesel fuels, you may have heard the term cetane number, which derives its name from hexadecane (trivial name: cetane), a straight-chain alkane with 16 carbons $\left(\mathrm{C}_{16} \mathrm{H}_{34}\right)$. The cetane number is a dimensionless indicator of the ignition quality of a diesel fuel and is conceptually similar to the better-known octane number used for gasoline. Hexadecane is the high-quality standard on the cetane scale and has been assigned a cetane number of 100 . A highly branched alkane, 2,2,4,4,6,8,8-heptamethylnonane (HMN; nine carbons in a chain with seven $\mathrm{CH}_{3}$ groups attached to it besides the two terminal $\mathrm{CH}_{3}$ groups, thus it also has 16 carbon atoms; try drawing its structure) is the low-quality compound on the cetane scale and has a cetane number of 15. Therefore, long, unbranched alkanes comprise an "ideal" petrodiesel fuel. The analogy to hexadecane as "ideal" petrodiesel component shows why biodiesel is suitable as an "alternative" diesel fuel. The fatty acids whose methyl esters are now used as biodiesel also are long-chain compounds similar to long-chain alkanes such as hexadecane which make good petrodiesel.

Petrodiesel consists of many components. Besides hydrocarbons such as those mentioned above, petrodiesel often contains significant amounts of compounds known as aromatics. Aromatics are cyclic compounds such as benzene or toluene (see structures in Figure 13). The carbon atoms are not shown here explicitly in the structures. They are assumed to be at the intersections of the lines that show the bonds between the carbons.

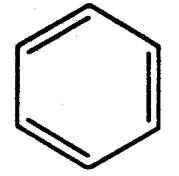

Benzene

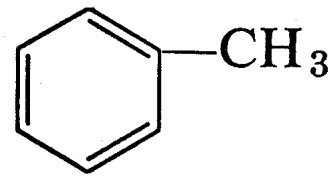

Toluene

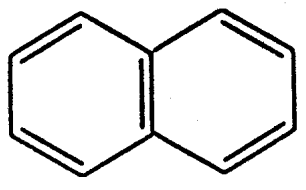

Naphthalene

Figure 13. Aromatic molecules

Thus, benzene has the formula $\mathrm{C}_{6} \mathrm{H}_{6}$ and toluene has the formula $\mathrm{C}_{7} \mathrm{H}_{8}$. The common feature of all aromatic compounds is such a ring structure. There are many aromatic compounds in which the ring structures are fused, for example, naphthalene (formula $\mathrm{C}_{10} \mathrm{H}_{8}$ ). They have low cetane numbers and therefore are undesirable components of petrodiesel. However, they have high densities and thus help elevate the energy contained in a gallon of the fuel. Biodiesel's lack of aromatic compounds is often cited as an advantage. 
Compounds in which several benzene rings are fused together (even more than in naphthalene) are termed polyaromatic hydrocarbons (PAHs). They are found in exhaust emissions of petrodiesel and, in reduced amounts, of biodiesel fuel.

Why are vegetable oils transesterified to produce biodiesel? This question will mainly be dealt with in other parts of the course(s), but briefly, vegetable oil methyl esters have lower viscosities (resistance to flow of a liquid) than the parent vegetable oils (think of honey or syrup, which have high viscosities and flow with difficulty, vs. water or milk, which have low viscosities and flow easily). Compared to the viscosities of the parent vegetable oils, the viscosities of vegetable oil methyl esters are much closer to that of petrodiesel. High viscosity causes operational problems in a diesel engine such as poor quality fuel injection and the formation of deposits.

\section{The Concept of Acids and Bases}

Many compounds, both organic and inorganic, can be classified as acids and bases. The most useful concept of acids and bases as these materials relate to biodiesel is that of proton donors (acids) or proton acceptors (bases), also often called Brønsted (or Lowry) acids and bases. Transferring a proton from an acid to a base is an acid-base reaction. A proton is a hydrogen atom stripped of its electron. Thus, a proton carries a positive charge and is often written $\mathrm{H}^{+}$.

Whether a compound can behave as an acid or base depends on some of the atoms contained in it. Thus, organic compounds containing nitrogen often have base character, while those containing oxygen in form of $\mathrm{OH}$ (hydroxy) groups often have acid character (there are compounds that have the characteristics of both but that will not concern us here). Also, there are acids and bases in which a proton has been replaced by another particle carrying a positive charge called a cation, derived from another element. Note that a charged particle, be it negatively or positively charged, is called an ion. Without going into further detail, that acids and bases split (dissociate) into ions instead of atoms results from the position of the atoms in that compound in the periodic table of the elements and the corresponding electron configuration of those atoms.

Thus, in water $\left(\mathrm{H}_{2} \mathrm{O}\right)$, one proton $\left(\mathrm{H}^{+}\right)$can be replaced by the positively charged cation of sodium $\left(\mathrm{Na}^{+}\right)$or potassium $\left(\mathrm{K}^{+}\right)$to give $\mathrm{NaOH}$ (sodium hydroxide) or $\mathrm{KOH}$ (potassium hydroxide). Obviously, the remaining hydroxy part $(\mathrm{OH})$ is then negatively charged to give $\mathrm{OH}^{-}$ (hydroxide). As a result, $\mathrm{NaOH}$ and $\mathrm{KOH}$ are strong bases as they have a tendency to take up a proton. Similarly, in an alcohol such as methanol $\left(\mathrm{CH}_{3} \mathrm{OH}\right)$, the proton attached to the oxygen atom can be replaced by, for example, $\mathrm{Na}^{+}$. The resulting $\mathrm{CH}_{3} \mathrm{ONa}$ (sodium methylate; sodium methoxide; sodium methanolate) is a strong base, $\mathrm{CH}_{3} \mathrm{O}^{-}$being the reason.

Similarly, but in the reverse fashion, there are acids, the best-known being sulfuric acid $\left(\mathrm{H}_{2} \mathrm{SO}_{4}\right)$, hydrochloric acid $(\mathrm{HCl})$ and nitric acid $\left(\mathrm{HNO}_{3}\right)$. Acids have a tendency to give off protons, leaving a negatively charged particle called an anion. For example, the anion of sulfuric acid is $\mathrm{HSO}_{4}^{-}$. 
After the above discussion, it is not too surprising that water can act as an acid and/or a base as water can be seen as $\mathrm{H}^{+}$and $\mathrm{OH}^{-}$giving $\mathrm{H}_{2} \mathrm{O}$. Actually, however, water reacts with itself as acid and base in the following fashion:

$$
\mathrm{H}_{2} \mathrm{O}+\mathrm{H}_{2} \mathrm{O} \rightarrow \mathrm{H}_{3} \mathrm{O}^{+}+\mathrm{OH}^{-}
$$

The double arrow indicates that a reaction can go in both directions. The concentrations of both $\mathrm{H}_{3} \mathrm{O}^{+}$and $\mathrm{OH}^{-}$in neutral water are very low. In chemical terminology it is then said that the equilibrium of the reaction is on the left side of the equation. (Also $\mathrm{H}_{2} \mathrm{O}+\mathrm{H}_{2} \mathrm{O}$ would usually be written as $2 \mathrm{H}_{2} \mathrm{O}$.)

The above reaction forms the basis of the important concept of the $\mathbf{p H}$ value. The $\mathrm{pH}$ value is defined as the concentration of $\mathrm{H}_{3} \mathrm{O}^{+}$(hydronium ion) in an aqueous solution.

It is very important to know that the $\mathrm{pH}$ of neutral water is 7 (this results from the concentration of $\mathrm{H}_{3} \mathrm{O}^{+}$in neutral water. (Question: How does the concentration of $\mathrm{OH}^{-}$in neutral water compare to that of $\mathrm{H}_{3} \mathrm{O}^{+}$?). An acidic solution has a $\mathrm{pH}$ below 7 while a basic solution has a $\mathrm{pH}$ above 7 .

The $\mathrm{pH}$ value of an aqueous solution can be conveniently measured directly in a matter of seconds using a pH meter. There are also strips of paper (indicator paper; a simpler version is litmus paper) that when held into a solution change color due to an acid-base reaction with the indicator on the paper. Comparison of the obtained color with a chart on the box in which the indicator paper is stored then gives the $\mathrm{pH}$.

Thus, an acid can be seen as a compound that produces $\mathrm{H}_{3} \mathrm{O}^{+}$when dissolved in water beyond the $\mathrm{H}_{3} \mathrm{O}^{+}$present in neutral water anyway. Conversely, a base produces $\mathrm{OH}^{-}$when dissolved in water beyond the $\mathrm{OH}^{-}$present in neutral water. (Exercise: Try writing the above equation for dissolving an acid or base in water. For an acid use the generic formula HA and for a base $\mathrm{A}^{-}$.)

Another important part of the acid-base chemistry is that of neutralization. Many chemical reactions, including the transesterification reaction that yields biodiesel, require the use of catalysts. Catalysts are compounds that are often present in small amounts that cause a reaction to proceed at a faster rate. Often such catalysts can be acids or bases. That means that even when the reaction is over, the reaction solution will still be acidic or basic, depending on the kind of catalyst used (and the other materials present). Then it is usually necessary to neutralize the solution, i.e., the solution needs to attain the neutral $\mathrm{pH}$. Not surprisingly, a basic solution can be neutralized with an acid and, vice versa, an acidic solution can be neutralized with a base. In many cases, a reaction of an acid and a base forms a salt, i.e., a product of part of the acid and base (the anion and cation). Actually, common table salt $(\mathrm{NaCl})$ is an example as it can be seen as arising from sodium hydroxide and hydrochloric acid: $\mathrm{NaOH}+\mathrm{HCl} \rightarrow \mathrm{NaCl}+\mathrm{H}_{2} \mathrm{O}$.

Many other salts are possible. When producing biodiesel under basic (more common) or acidic (less common) conditions, neutralization is required. That neutralization may lead to a salt, that, although a side product of the process, may even be sold. It may be worthy of consideration to choose the neutralizing acid (or base) based not only on its cost but also on the market potential 
of the salt. However, attention is required to possible contamination of the glycerol by the salt, which may influence the purification, applicability, and market potential of the glycerol. The salts may possess different properties, including retention, relative to glycerol.

For application of the acid-base concept to the organic chemistry of fats and oils, biodiesel, and the transesterification reaction, it is important to recall that fatty acids are acids (organic acids are often termed carboxylic acids), although rather weak ones. The important functionality in the fatty acid molecule is $-\mathrm{COOH}$. The proton can dissociate to give $-\mathrm{COO}^{-}$and $\mathrm{H}^{+}$. Thus fatty acids can also form salts, in which the $\mathrm{H}^{+}$is replaced by some cation such as $\mathrm{Na}^{+}$or $\mathrm{K}^{+}$. Such salts of fatty acids form the basis of soaps, as described earlier.

Fatty acids can also form esters, i.e. react with an alcohol, for example, methanol:

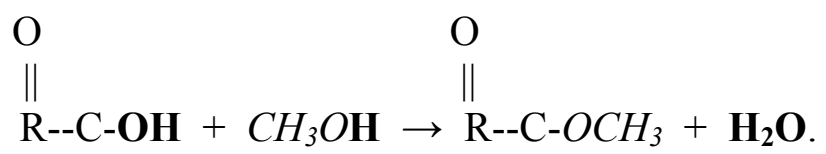

The italicized and bolded parts of the equation indicate which parts are exchanged. Actually in the esterification of the acid with methanol, it is $\mathrm{OH}^{-}$that is exchanged for $\mathrm{CH}_{3} \mathrm{O}^{-}$. Thus, under the right conditions, a carboxylic acid can give off either $\mathrm{H}^{+}$or $\mathrm{OH}^{-}$. It is also possible to transform one ester into another:

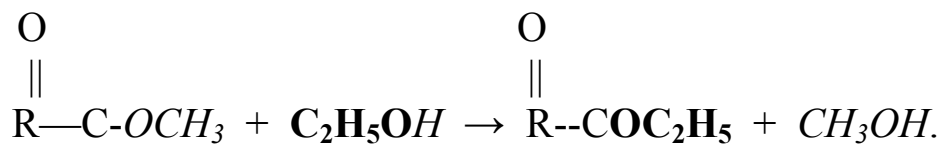

However as most carboxylic acids, including fatty acids, and alcohols are very weak acids and bases, stronger acids or bases need to be added to their mixture to achieve a reaction. These acids or bases function as the catalysts.

\section{Catalysts}

Two of the most commonly used catalysts for transesterification are $\mathrm{NaOH}$ and $\mathrm{KOH}$. These catalysts operate by reacting with the alcohol according to the reaction given below (written using methanol and $\mathrm{NaOH}$ but other alcohols and catalysts could be substituted).

$$
\mathrm{CH}_{3} \mathrm{OH}+\mathrm{NaOH} \rightarrow \mathrm{CH}_{3} \mathrm{O}-\mathrm{Na}+\mathrm{H}_{2} \mathrm{O}
$$

Similar to $\mathrm{H}_{2} \mathrm{O}$ "consisting" of $\mathrm{H}^{+}$and $\mathrm{OH}^{-}, \mathrm{CH}_{3} \mathrm{O}-\mathrm{Na}$ can be seen as consisting of $\mathrm{CH}_{3} \mathrm{O}^{-}$(alkoxide; alkylate) and $\mathrm{Na}^{+} . \mathrm{CH}_{3} \mathrm{O}^{-}$is the species that attacks the ester moieties in the glycerol molecule in the following fashion: 


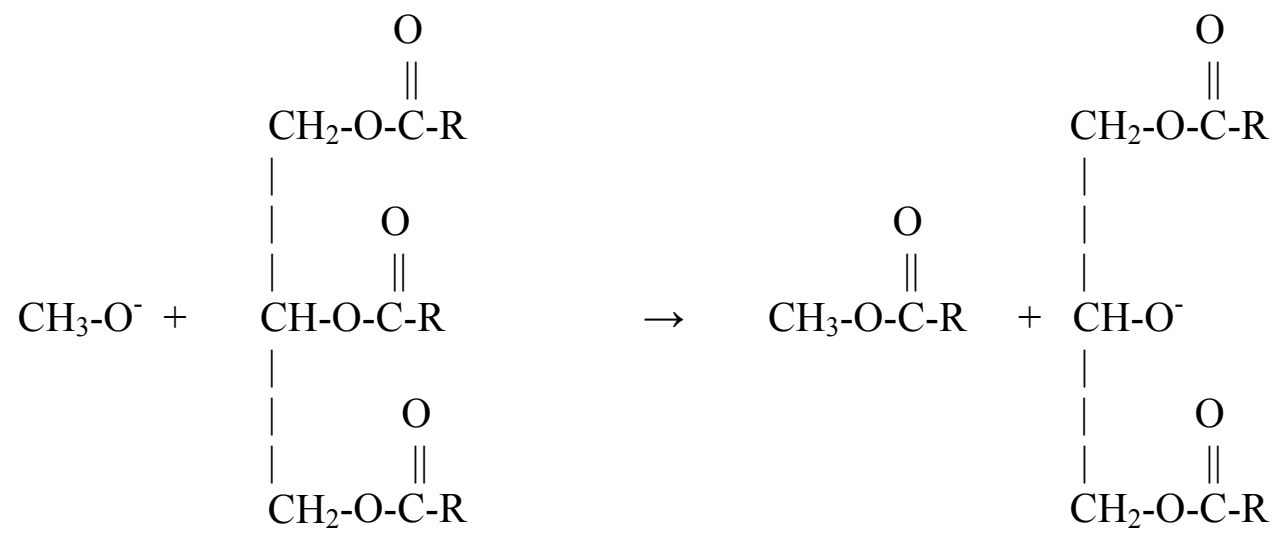

(Eq. 2)

\section{Alkoxide Triglyceride Methyl ester Triglyceride anion}

While the ester molecule is complete after this reaction, the anion of the triglyceride needs to pick up a proton to give a stable product (a diglyceride in this case). If this proton is taken from methanol, then the alkoxide catalyst will be recovered as shown in Eq. 3.

Several other reactions could be written that would allow the anion of the triglyceride to pick up a proton, such as reaction with free fatty acids and water. The presence of water affects the transesterification negatively because the triglyceride anion in Eq. 3 will react

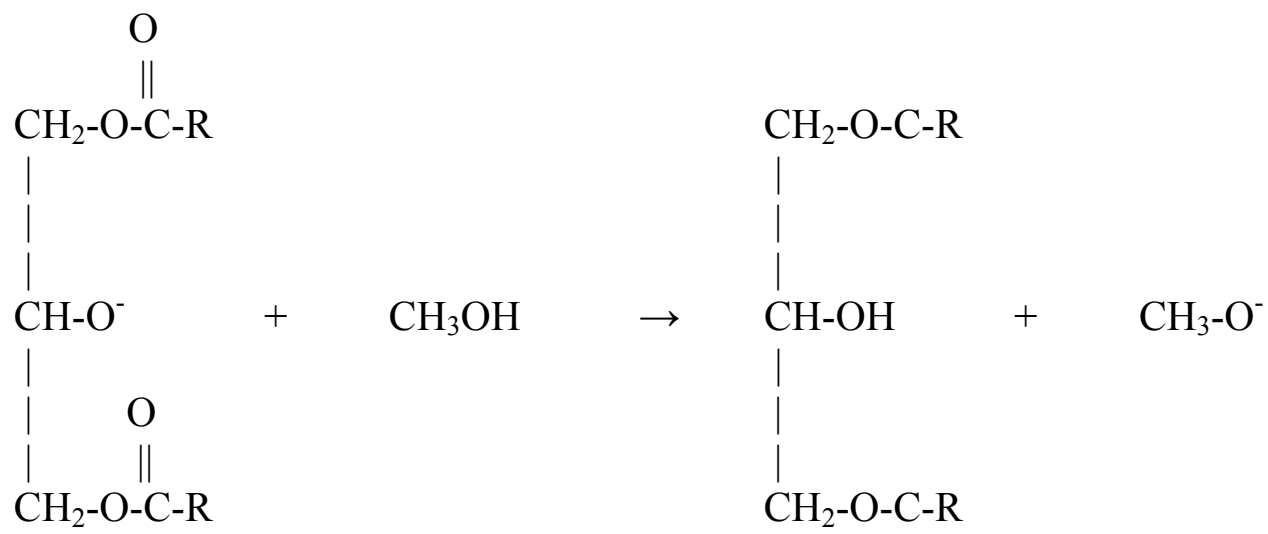

with the water to form $\mathrm{OH}^{-}$, which can behave in a fashion similar to $\mathrm{CH}_{3}-\mathrm{O}^{-}$but a free fatty acid will result instead of a methyl ester. 


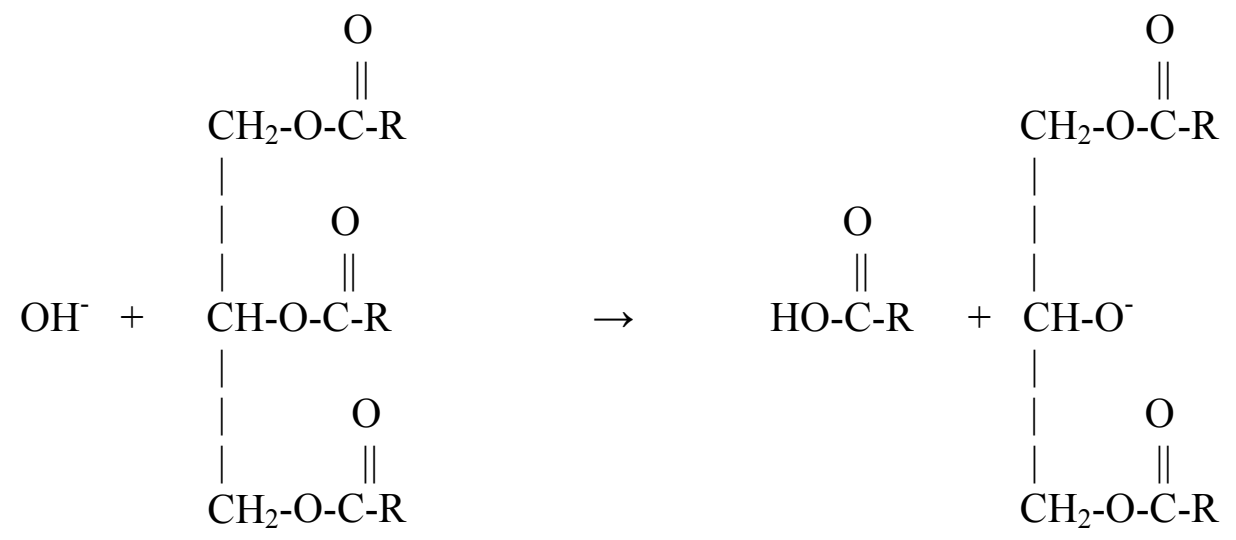

(Eq. 4)

Hydroxide Triglyceride

Free fatty acid

Triglyceride anion

The free fatty acid can react with the $\mathrm{Na}^{+}$to form soap. Some water in the system can be tolerated, because $\mathrm{R}^{-} \mathrm{O}^{-}$is a stronger base than $\mathrm{OH}^{-}$so the transesterification reaction occurs at a higher rate than the "saponification" of glycerol leading to free fatty acids. The equilibrium of the following reaction (formation of free fatty acids from the ester) is far on the left side of the reaction equation:

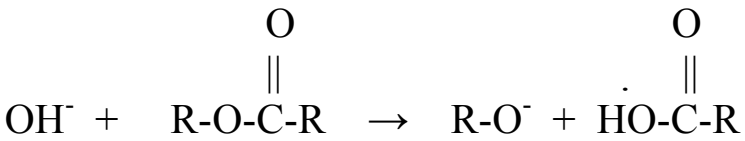

$$
\begin{aligned}
& \text { alkyl ester free fatty acid }
\end{aligned}
$$

As a result of the slow reaction rate, only very minor amounts of free fatty acids are formed during transesterification if the reaction is free of water at the beginning. Another aspect of the weaker basicity of $\mathrm{OH}^{-} v s$. $\mathrm{R}^{-} \mathrm{O}^{-}$is that the ester moieties in the triglyceride molecules will react less with $\mathrm{OH}^{-}$than $\mathrm{R}^{-} \mathrm{O}^{-}$. However, when too much catalyst (or water) is present, Eq. 5 becomes more prevalent and causes enhanced formation of mono- and diglyceride molecules instead of reactions with all positions in the glycerol backbone. Thus, the effect of too much catalyst or too much water leads to the same result, namely, enhanced formation of undesirable mono- and diglycerides as well as free fatty acids. Too much catalyst can lead to soap formation when conditions encourage free fatty acid production.

Because of the possibility of the reactions described above leading to free fatty acids and monoand diglycerides, direct use of sodium or potassium alkylate (R-ONa or R-OK; the alkylate moiety must correspond to the R- moiety in the alcohol) as catalysts is becoming of greater interest. The reaction of alcohol and $\mathrm{XOH}$ given above (Eq. 1) cannot occur in this case (assuming, of course, that the reaction system is free of water). Instead, the transesterification according to Eq. 2 can occur directly. 


\section{Biodiesel Specifications and Properties}

\section{Introduction}

This module will acquaint you with the fuel specification that defines and sets the quality standards for biodiesel. The standard is framed as a set of property specifications measured by specific ASTM test methods. The standard for biodiesel is ASTM 6751-02.

ASTM D 6751 - 02 sets forth the specifications that must be met for a fatty acid ester product to carry the designation "biodiesel fuel" or "B100"or for use in blends with any petroleum-derived diesel fuel defined by ASTM D 975, Grades 1-D, 2-D, and low sulfur 1-D and 2-D.

The instructional goals for this module are:

1. Learn the Specifications for B 100 fuel.

2. Introduce the Methods used to measure the performance parameters for B 100 fuel.

3. Describe the Methods and measurements needed for a basic quality control laboratory for a production facility.

\section{Definition of "Biodiesel"}

Biodiesel is defined as: a fuel comprised of mono-alkyl esters of long chain fatty acids derived from vegetable oils or animal fats, designated B100.

A "mono-alkyl ester" is the product of the reaction of a straight chain alcohol, such as methanol or ethanol, with a fat or oil (triglyceride) to form glycerol (glycerin) and the esters of long chain fatty acids.

Biodiesel can be used as B 100 (neat) or in a blend with petroleum diesel. A blend of $20 \%$ biodiesel with $80 \%$ petrodiesel, by volume, is termed "B 20". A blend of $2 \%$ biodiesel with 98 $\%$ petrodiesel is " $\mathrm{B} 2$ ", and so on.

\section{Property Requirements and Specified Methods for B100}

The values of the various biodiesel properties specified by ASTM D 6751 are listed in Table 6. Each of these properties and the test method used to measure it are described below.

\section{Method: ASTM D93- Flash point, closed cup Requirement: $130^{\circ} \mathrm{C}$ min}

The flash point is defined as the "lowest temperature corrected to a barometric pressure of 101.3 $\mathrm{kPa}(760 \mathrm{~mm} \mathrm{Hg})$, at which application of an ignition source causes the vapors of a specimen to ignite under specified conditions of test." This test, in part, is a measure of residual alcohol in the B100. 
Table 6. ASTM D 6751 - 02 Requirements

\begin{tabular}{|c|c|c|c|}
\hline Property & Method & Limits & Units \\
\hline Flash point, closed cup & D 93 & 130 min & ${ }^{\circ} \mathrm{C}$ \\
\hline Water and sediment & D 2709 & 0.050 max & $\%$ volume \\
\hline Kinematic viscosity, $40^{\circ} \mathrm{C}$ & D 445 & $1.9-6.0$ & $\mathrm{~mm}^{2} / \mathrm{s}$ \\
\hline Sulfated ash & D 874 & $0.020 \mathrm{max}$ & wt. \% \\
\hline Total Sulfur & D 5453 & 0.05 max & wt. $\%$ \\
\hline Copper strip corrosion & D 130 & No. 3 max & \\
\hline Cetane number & D 613 & 47 min & \\
\hline Cloud point & D 2500 & Report to customer & ${ }^{\circ} \mathrm{C}$ \\
\hline Carbon residue & D 4530 & 0.050 max & wt. $\%$ \\
\hline Acid number & D 664 & 0.80 max & mg KOH/g \\
\hline Free glycerin & D 6584 & 0.020 & wt. \% \\
\hline Total glycerin & D 6584 & 0.240 & wt. \% \\
\hline Phosphorus & D 4951 & 0.0010 & wt. $\%$ \\
\hline Vacuum distillation end point & D 1160 & $360{ }^{\circ} \mathrm{C} \mathrm{max,} \mathrm{at}$ & ${ }^{\circ} \mathrm{C}$ \\
\hline Storage stability & $\begin{array}{c}\text { To be } \\
\text { determined }\end{array}$ & To be determined & $\begin{array}{c}\text { To be } \\
\text { determined }\end{array}$ \\
\hline
\end{tabular}

The flash point is a determinant for flammability classification of materials. The typical flash point of pure methyl esters is $>200^{\circ} \mathrm{C}$, classifying them as "non-flammable". However, during production and purification of biodiesel, not all the methanol may be removed, making the fuel flammable and more dangerous to handle and store if the flash point falls below $130^{\circ} \mathrm{C}$. Excess methanol in the fuel may also affect engine seals and elastomers and corrode metal components. Generally, a production Quality Control (QC) laboratory should include a flash point apparatus for quality control and as a means of detecting excess alcohol levels.

\section{Method: ASTM D 2709 - Water and sediment Requirement: $0.050 \%$ volume $\max$}

Water and sediment is a test that "determines the volume of free water and sediment in middle distillate fuels having viscosities at $40{ }^{\circ} \mathrm{C}$ in the range 1.0 to $4.1 \mathrm{~mm} 2 / \mathrm{s}$ and densities in the range of 700 to $900 \mathrm{~kg} / \mathrm{m} 3$."

This test is a measure of cleanliness of the fuel. For B100 it is particularly important because water can react with the esters, making free fatty acids, and can support microbial growth in storage tanks. Water is usually kept out of the production process by removing it from the feedstocks. However, some water may be formed during the process by the reaction of the sodium or potassium hydroxide catalyst with alcohol. If free fatty acids are present, water will be formed when they react to either biodiesel or soap. Finally, water is deliberately added during the 
washing process to remove contaminants from the biodiesel. This washing process should be followed by a drying process to ensure the final product will meet ASTM D 2709.

Sediments may plug fuel filters and may contribute to the formation of deposits on fuel injectors and other engine damage. Sediment levels in biodiesel may increase over time as the fuel degrades during extended storage. The production QC lab should be equipped to perform this test on a routine basis.

\section{Method: ASTM D 445 - Kinematic viscosity, $40^{\circ} \mathrm{C}$ Requirement: $1.9-6.0 \mathrm{~mm}^{2} / \mathrm{s}$}

Kinematic viscosity: "the resistance to flow of a fluid under gravity". [The kinematic viscosity is equal to the dynamic viscosity/density] The kinematic viscosity is a basic design specification for the fuel injectors used in diesel engines. Too high a viscosity, and the injectors do not perform properly.

Dynamic viscosity - 'ratio between applied shear stress and rate of shear of a liquid."

Density - "the mass per unit volume of a substance at a given temperature."

The viscosity of biodiesel can be predicted $\pm 15 \%$ using the esters composition determined using ASTM D 6584. The viscosity apparatus to run D 445 is not critical to the QC lab, but it is valuable as a quick assay method for estimating the degree of completion for a reaction batch.

\section{Method: ASTM D 874-Sulfated Ash \\ Requirement: 0.020 wt. \%, $\max$}

Sulfated ash is the "... residue remaining after a [fuel] sample has been carbonized, and the residue subsequently treated with sulfuric acid and heated to a constant weight." This test monitors the mineral ash residual when a fuel is burned.

For biodiesel, this test is an important indicator of the quantity of residual metals in the fuel that came from the catalyst used in the esterification process. Producers that use a base catalyzed process may wish to run this test regularly. Many of these spent sodium or potassium salts have low melting temperatures and may cause engine damage in combustion chambers.

\section{Method: ASTM D5453 - Total sulfur \\ Requirement: 0.05 wt. \%, $\max$}

"This method covers the determination of total sulfur in liquid hydrocarbons, boiling in the range from approximately 25 to $400^{\circ} \mathrm{C}$, with viscosities between approximately 0.2 and $20 \mathrm{cSt}$ $(\mathrm{mm} 2 / \mathrm{s})$ at room temperature."

Biodiesel feedstocks typically have very little sulfur, but this test is an indicator of contamination of protein material and/or carryover catalyst material or neutralization material from the production process. Producers using rendered or waste feedstocks should have access to this measurement for their feedstocks. Some biodiesels from rendered fats and greases have been 
found to have sulfur levels of $40-50 \mathrm{ppm}$. The sulfur limits for on-highway diesel fuel will be reduced to $0.0015 \%$ (15 ppm) in 2006, so producers that do not meet this future specification on a regular basis may need to consider sulfur removal technology in their facility.

\section{Method: ASTM D 130 - Copper strip corrosion Requirement: No. 3, $\max$}

The copper strip corrosion is used " [ for the] detection of the corrosiveness to copper of fuels and solvents." This test monitors the presence of acids in the fuel.

For B 100, the most likely source of a test failure would be excessive free fatty acids, which are determined in accordance with an additional specification. The producer may choose to run this test periodically, but the acid number (D 664) determination is the more important acid content QC measurement.

\section{Method: ASTM D 613 - Cetane number Requirement: 47 , $\min$}

The cetane number is "a measure of the ignition performance of a diesel fuel obtained by comparing it to reference fuels in a standardized engine test." Cetane for diesel engines is analogous to the octane rating in a spark ignition engine - it is a measure of how easily the fuel will ignite in the engine.

For B100, the cetane number is seldom an issue because all of the common fatty acid esters have cetane numbers near or above 47 . The cetane number can be predicted $\pm 10 \%$ using the esters composition. It is unlikely that an individual producer will ever run cetane tests on-site because the equipment is extremely expensive.

\section{Method: ASTM D 2500 - Cloud point Requirement: Report in ${ }^{\circ} \mathrm{C}$ to customer}

The cloud point is "The temperature at which a cloud of wax crystals first appears in a liquid when it is cooled down under conditions prescribed in this test method." The cloud point is a critical factor in cold weather performance for all diesel fuels.

The chemical composition of some biodiesel feedstocks leads to a B100 that may have higher cloud points than customers desire. The cloud point, however, is another parameter that can be predicted $\pm 5 \%$ with knowledge of the esters composition, but producers are advised to be equipped to perform this test. Since the saturated methyl esters are the first to precipitate, the amounts of these esters, methyl palmitate and methyl stearate, are the determining factors for the cloud point.

The producer can modify the cloud point two ways. One is through the use of additives that retard the formation of solid crystals in the B100 by various mechanisms. The cloud point can also be modified by blending feedstocks that are relatively high in saturated fatty acids with feedstocks that have a lower saturated fatty acid content. The result is a net lower cloud point for 
the mixture. See the section on prediction of physical properties for a more complete discussion of the effect of composition on cloud point.

Method: ASTM D 4530 - Carbon residue

Requirement: $0.050 \mathrm{wt} \%$, $\max$

"In petroleum products, the part remaining after a sample has been subjected to thermal decomposition..." is the carbon residue. The carbon residue is a measure of how much residual carbon remains after combustion. The test basically involves heating the fuel to a high temperature in the absence of oxygen. Most of the fuel will vaporize and be driven off, but a portion may decompose and pyrolyze to hard carbonaceous deposits. This is particularly important in diesel engines because of the possibility of carbon residues clogging the fuel injectors.

The most common cause of excess carbon residues in B 100 is an excessive level of total glycerin. Total glycerin is also measured directly using ASTM D 6584, so this measurement is generally not critical to the producer.

\section{Method: ASTM D 664 - Acid number Requirement: $0.80 \mathrm{mg} \mathrm{KOH} / \mathrm{g}, \max$}

The acid number is "The quantity of base, expressed as milligrams of potassium hydroxide per gram of sample, required to titrate a sample to a specified end point." The acid number is a direct measure of free fatty acids in B100. The free fatty acids can lead to corrosion and may be a symptom of water in the fuel. Usually, for a base catalyzed process, the acid value after production will be low since the base catalyst will strip the available free fatty acids. However, the acid value may increase with time as the fuel degrades due to contact with air or water. This test should be performed regularly as a part of the producer QC program.

\section{Method: ASTM D 6584 - Free glycerine Requirement: 0.020 wt. \%}

Free glycerol is the glycerol present as molecular glycerol in the fuel. Free glycerol results from incomplete separation of the ester and glycerol products after the transesterification reaction. This can be a result of imperfect water washing or other approaches that do not effectively separate the glycerol from the biodiesel. The free glycerol can be a source of carbon deposits in the engine because of incomplete combustion. The terms "glycerine," "glycerin," and "glycerol" are used interchangeably.

\section{Method: ASTM D 6584 - Total glycerine Requirement: 0.240 wt. \%}

Total glycerol "is the sum of free and bonded glycerol." Bonded glycerol "is the glycerol portion of the mono-, di-, and triglyceride molecules." Elevated total glycerol values are indicators of incomplete esterification reactions and predictors of excessive carbon deposits in the engine. 
The ASTM D 6584 test for free and total glycerol must be done routinely as the key measurement in the producer's QC program.

\section{Method: ASTM D 4951 - Phosphorus Requirement: 0.0010 wt. \%}

"This test covers the quantitative determination of barium, calcium, copper, magnesium, phosphorus, sulfur, and zinc in unused lubricating oils and additive packages." In the case of B100, phosphorus can come from incomplete refining of the phospholipids (or gums) from the vegetable oil and from bone and proteins encountered in the rendering process. The producer should have access to this method for periodic measurements.

\section{Method: ASTM D 1160 - Vacuum distillation end point Requirement: $360^{\circ} \mathrm{C}$ max, at $90 \%$ liquid distilled}

The vacuum distillation end-point test "covers the determination, at reduced pressures, of the range of boiling points for petroleum products that can be partially or completely vaporized at a maximum liquid temperature of $400^{\circ} \mathrm{C}$."

Petroleum fractions have tens to hundreds of individual compounds mixed together. The distillation curves are used to characterize the broad chemistry of a given crude oil source in terms of the boiling temperatures of its constituent compounds.

In B100 there are, at most, ten different esters present, and they can be identified using gas or liquid chromatography. The same chromatograph that determines free and total glycerin can determine the esters composition in the B100. This composition allows calculation of the T-90 point for the fuel, without having to perform the testing for every batch of product. It is unlikely that the producer would have a reason to run this test except to certify compliance with the ASTM standard.

\section{Storage stability - Method to be determined}

All fuels are subject to degradation over time when they are stored. This degradation may be due to microbial action, water intrusion, air oxidation, etc. The standard and the test methods for determining storage stability for B100 are still in the development stage within the ASTM process. In general, the following parameters will change and can be used to determine if the fuel should not be used: acid number, water and sediment, and viscosity. Should an aged fuel fail any of these three standards, it should not be used.

\section{Sources for ASTM Standards}

Complete copies for the ASTM Standards and Methods referenced above are available from ASTM by mail, fax order, or on-line purchase for $\$ 35.00$ per standard. Most universities that have an engineering college, and many larger libraries, include ASTM Standards in hard copy form in their reference collection.

The Standards and Methods are very detailed and some are quite complex. A number of the Methods include reference to additional Methods, so the overall library needed for a QC lab is 
rather large. The methods are revised occasionally, so it is prudent to ensure that your lab has the most up-to-date revisions annually.

\section{QC Laboratory Recommendations}

The QC Laboratory is an essential element of any biodiesel production facility. An individual trained in chemistry through the level of organic chemistry, analytical chemistry, and instrumental analysis is recommended for the QC chemist function. Most community colleges offer training in an Associates Degree program that will meet these criteria. Some of the methods used are quite specialized, so training in the conduct of the methods is also recommended. Table 7 shows a listing of the specific test methods that are recommended for use in the plant laboratory.

\section{Summary}

ASTM Specification D 6751 - 02, specification for B 100 (biodiesel) fuel is the basis for ensuring that quality products are provided to the fuel distribution system. The key properties of B 100 are discussed in terms of their Test Methods and specifications.

Essential testing capabilities for the producer include:

- ASTM D 2709, Water and Sediment;

- ASTM D 874, Sulfated Ash;

- ASTM D 2500, Cloud Point;

- ASTM D 664, Acid Number, and;

- ASTM D 6584, Free Glycerine and Total Glycerine 
Table 7. QC Laboratory Recommended Equipment: Needs and Costs

\begin{tabular}{|l|l|l|}
\hline ASTM Method & \multicolumn{1}{|c|}{ Apparatus } & \multicolumn{1}{|c|}{$\begin{array}{c}\text { Approx. } \\
\text { Cost }\end{array}$} \\
\hline & \multicolumn{1}{|c|}{} & \\
\hline D93 - Flash Point & Hertzog MP 329 Automatic Pensky-Martens Tester & $\$ 10,300$ \\
\hline & Koehler Pensky-Martens Flash Cup Tester & $\$ 2,200$ \\
\hline D130 - Corrosion & Koehler K25330 Copper Strip Test Bath & $\$ 3,700$ \\
\hline & Standards: ASTM - \$ 195; Koehler - \$ 466 & \\
\hline D445 - Viscosity & Koehler AKV9500 Automated Kinematic Viscosity System & $\$ 22,500$ \\
\hline & Polystat Constant Temperature Bath (Cole - Parmer) & $\$ 2,900$ \\
\hline & Viscometer Cleaning and Drying Apparatus (Koehler) & $\$ 3,400$ \\
\hline D664 - Acid Value & Viscometers, various ranges & $\$ 120$ ea \\
\hline & KEM AT-150 Automatic Potentiometric Titrator & $\$ 7,000$ \\
\hline $\begin{array}{l}\text { D874 - Sulfated } \\
\text { Ash }\end{array}$ & Koehler Isotemp Basic Muffle Furnace, 0.58 cu.ft. & $\$ 2,500$ \\
\hline $\begin{array}{l}\text { D2500 - Cloud } \\
\text { Point }\end{array}$ & Koehler Cloud and Pour Point Bath & $\$ 5,500$ \\
\hline & Koehler Automated Cloud and Pour Point System & $\$ 21,000$ \\
\hline $\begin{array}{l}\text { D2709 - Water and } \\
\text { Sediment }\end{array}$ & PAC 67310 Benchtop Centrifuge & $\$ 6,600$ \\
\hline $\begin{array}{l}\text { D6584 - Total and } \\
\text { Free Glycerol }\end{array}$ & Shimadzu GC-17A Gas Chromatograph & $\$ 18,000$ \\
\hline \multicolumn{1}{|c|}{ Total Cost } & Recommended Apparatus & \\
\hline
\end{tabular}




\section{Types of Biodiesel Production Processes}

\section{Types of Biodiesel Production Processes}

\section{Introduction}

This module provides an overview of the steps in the production of biodiesel from preparation of the feedstock to the recovery and purification of the fatty acid esters (biodiesel) and the coproduct glycerol (also called glycerin). We will review several chemistries used for esterification and different approaches to product preparation and purification.

The emphasis throughout the module will be the choices to be made in matching feedstock selection, capacity, and operating mode with the selection of the basic process chemistry and layout for a specific location. While no specific process technology is favored in this description, an effort has been made to describe the major approaches currently in use in the industry.

\section{Feedstocks Used in Biodiesel Production}

The primary raw materials used in the production of biodiesel are vegetable oils, animal fats, and recycled greases. These materials contain triglycerides, free fatty acids, and other contaminants depending on the degree of pretreatment they have received prior to delivery. Since biodiesel is a mono-alkyl fatty acid ester, the primary alcohol used to form the ester is the other major feedstock.

Most processes for making biodiesel use a catalyst to initiate the esterification reaction. The catalyst is required because the alcohol is sparingly soluble in the oil phase. The catalyst promotes an increase in solubility to allow the reaction to proceed at a reasonable rate. The most common catalysts used are strong mineral bases such as sodium hydroxide and potassium hydroxide. After the reaction, the base catalyst must be neutralized with a strong mineral acid.

Typical proportions for the chemicals used to make biodiesel are:

$$
\begin{array}{ll}
\text { Reactants } & \text { •Fat or oil (e.g. } 100 \mathrm{~kg} \text { soybean oil) } \\
& \text {-Primary alcohol (e.g. } 10 \mathrm{~kg} \text { methanol) } \\
\text { Catalyst } & \text { •Mineral base (e.g. } 0.3 \mathrm{~kg} \text { sodium hydroxide) } \\
\text { Neutralizer } & \text { •Mineral acid (e.g. } 0.25 \mathrm{~kg} \text { sulfuric acid) }
\end{array}
$$

Fats and Oils: Choice of the fats or oils to be used in producing biodiesel is both a process chemistry decision and an economic decision. With respect to process chemistry, the greatest difference among the choices of fats and oils is the amount of free fatty acids that are associated with the triglycerides. Other contaminants, such as color and odor bodies can reduce the value of the glycerin produced, and reduce the public acceptance of the fuel if the color and odor persist in the fuel.

Most vegetable oils have a low percentage of associated free fatty acids. Crude vegetable oils contain some free fatty acids and phospholipids. The phospholipids are removed in a 
"degumming" step and the free fatty acids are removed in a "refining" step. Oil can be purchased as crude, degummed, or refined. The selection of the type of oil affects the production technology that is required.

Animal tallows and recycled (yellow) grease have much higher levels of free fatty acids. Yellow grease is limited to $15 \%$ free fatty acids and is a traded commodity that is typically processed into animal and pet food. Specifications for yellow grease are described in the chapter on Feedstock Preparation. Trap greases come from traps under kitchen drains and these greases can contain between 50 and $100 \%$ free fatty acids. There is no market for these greases at this time and most are landfilled. Trap grease is not yet used for biodiesel production and may have some technical challenges that have not be fully resolved such as difficult to break emulsifications (gels), fine silt that will cause equipment wear, high water contents, and very strong color and odor bodies that affect biodiesel and glycerin products. There are also unresolved questions about small quantities of other contaminants such as pesticides that might be present in the fuel.

The options for the triglyceride choice are many. Among the vegetable oils sources are soybean, canola, palm, and rape. Animal fats are products of rendering operations. They include beef tallow, lard, poultry fat, and fish oils. Yellow greases can be mixtures of vegetable and animal sources. There are other less desirable, but also less expensive triglyceride sources such as brown grease and soapstock. The free fatty acid content affects the type of biodiesel process used, and the yield of fuel from that process. The other contaminants present can affect the extent of feedstock preparation necessary to use a given reaction chemistry.

Alcohol: The most commonly used primary alcohol used in biodiesel production is methanol, although other alcohols, such as ethanol, isopropanol, and butyl, can be used. A key quality factor for the primary alcohol is the water content. Water interferes with transesterification reactions and can result in poor yields and high levels of soap, free fatty acids, and triglycerides in the final fuel. Unfortunately, all the lower alcohols are hygroscopic and are capable of absorbing water from the air.

Many alcohols have been used to make biodiesel. As long as the product esters meet ASTM 6751 , it does not make any chemical difference which alcohol is used in the process. Other issues such as cost of the alcohol, the amount of alcohol needed for the reaction, the ease of recovering and recycling the alcohol, fuel tax credits, and global warming issues influence the choice of alcohol. Some alcohols also require slight technical modifications to the production process such as higher operating temperatures, longer or slower mixing times, or lower mixing speeds.

Since the reaction to form the esters is on a molar basis and we purchase alcohol on a volume basis, their properties make a significant difference in raw material price. It takes three moles of alcohol to react completely with one mole of triglyceride. Today, one gallon of methanol costs $\$ 0.61$. That gallon contains 93.56 gram-moles of methanol; at a cost of $\$ 0.00652$ per grammole. By contrast, a gallon of ethanol, at the current price of $\$ 1.45$ per gallon for fuel-grade ethanol, costs $\$ 0.02237$ per gram-mole, or 3.4 times more.

In addition, a base catalyzed process typically uses an operating mole ratio of 6:1 mole of alcohol rather than the 3:1 ratio required by the reaction. The reason for using extra alcohol is 
that it "drives" the reaction closer to the $99.7 \%$ yield we need to meet the total glycerol standard for fuel grade biodiesel. The unused alcohol must be recovered and recycled back into the process to minimize operating costs and environmental impacts. Methanol is considerably easier to recover than the ethanol. Ethanol forms an azeotrope with water so it is expensive to purify the ethanol during recovery. If the water is not removed it will interfere with the reactions. Methanol recycles easier because it doesn't form an azeotrope.

These two factors are the reason that even though methanol is more toxic, it is the preferred alcohol for producing biodiesel. Methanol has a flash point of $10{ }^{\circ} \mathrm{C}$, while the flash point of ethanol is $8^{\circ} \mathrm{C}$, so both are considered highly flammable. You should never let methanol come into contact with your skin or eyes as it can be readily absorbed. Excessive exposure to methanol can cause blindness and other health effects. For student demonstrations, ethanol may be safer to use.

Methanol does have a somewhat variable pricing structure. When the production of MTBE was mandated for the reduction of emissions from gasoline engines in the winter, there was a significant expansion in world capacity for the material. The excess capacity and crash in demand led to methanol prices of \$ 0.31 per gallon in early 2002. However, in late July, 2002, the production/consumption levels regained equilibrium and the methanol price doubled back to the more typical value of $\$ 0.60 \pm$ per gallon.

The alcohol quality requirements are that it be un-denatured and anhydrous. Since chemical grade ethanol is typically denatured with poisonous material to prevent its abuse, finding undenatured ethanol is difficult. Purchase ethanol that has been denatured with methanol if possible.

Catalysts and Neutralizers:

Catalysts may either be base, acid, or enzyme materials. The most commonly used catalyst materials for converting triglycerides to biodiesel are sodium hydroxide, potassium hydroxide, and sodium methoxide. Most base catalyst systems use vegetable oils as a feedstock. If the vegetable oil is crude, it contains small amounts $(<2 \%)$ of free fatty acids that will form soaps that will end up in the crude glycerin. Refined feedstocks, such as refined soy oil can also be used with base catalysts.

The base catalysts are highly hygroscopic and they form chemical water when dissolved in the alcohol reactant. They also absorb water from the air during storage. If too much water has been adsorbed the catalyst will perform poorly and the biodiesel may not meet the total glycerin standard.

Although acid catalysts can be used for transesterification they are generally considered to be too slow for industrial processing. Acid catalysts are more commonly used for the esterification of free fatty acids. Acid catalysts include sulfuric acid and phosphoric acid. Solid calcium carbonate is used as an acid catalyst in one experimental homogeneous catalyst process. The acid catalyst is mixed with methanol and then this mixture is added to the free fatty acids or a feedstock that contains high levels of free fatty acids. The free fatty acids convert into biodiesel. The acids will need neutralization when this process is complete, but this can be done as base catalyst is added to convert any remaining triglycerides. 
There is continuing interest in using lipases as enzymatic catalysts for the production of alkyl fatty acid esters. Some enzymes work on the triglyceride, converting them to methyl esters; and some work on the fatty acids. The commercial use of enzymes is currently limited to countries like Japan, where energy costs are high, or for the production of specialty chemicals from specific types of fatty acids. The commercial use of enzymes is limited because costs are high, the rate of reaction is slow, and yields to methyl esters are typically less than the $99.7 \%$ required for fuel-grade biodiesel. Enzymes are being considered for fatty acid conversion to biodiesel as a pretreatment step, but this system is not commercial at this time.

Neutralizers are used to remove the base or acid catalyst from the product biodiesel and glycerol. If you are using a base catalyst, the neutralizer is typically an acid, and visa versa. If the biodiesel is being washed, the neutralizer can be added to the wash water. While hydrochloric acid is a common choice to neutralize base catalysts, as mentioned earlier, if phosphoric acid is used, the resulting salt has value as a chemical fertilizer.

Catalyst Selection: Base catalysts are used for essentially all vegetable oil processing plants. The initial free fatty acid content and the water content are generally low. Tallows and greases with free fatty acid contents greater than about $1 \%$ must be pretreated to either remove the FFA or convert the FFA to esters before beginning the base catalyzed reaction. Otherwise, the base catalyst will react with the free fatty acids to form soap and water. The soap formation reaction is very fast and goes to completion before any esterification begins.

Essentially all of the current commercial biodiesel producers use base catalyzed reactions. Base catalyzed reactions are relatively fast, with residence times from about 5 minutes to about 1 hour, depending on temperature, concentration, mixing and alcohol:triglyceride ratio. Most use $\mathrm{NaOH}$ or $\mathrm{KOH}$ as catalysts, although glycerol refiners prefer $\mathrm{NaOH}$. $\mathrm{KOH}$ has a higher cost but the potassium can be precipitated as $\mathrm{K} 3 \mathrm{PO} 4$, a fertilizer, when the products are neutralized using phosphoric acid. This can make meeting water effluent standards a bit more difficult because of limits on phosphate effluents.

Sodium methoxide, usually as a $25 \%$ solution in methanol, is a more powerful catalyst on a weight basis than the mixture of $\mathrm{NaOH}$ and methanol. This appears to be, in part, the result of the negative effect of the chemical water produced in situ when $\mathrm{NaOH}$ and methanol react to form sodium methoxide.

Acid catalyst systems are characterized by slow reaction rates and high alcohol:TG requirements (20:1 and more). Generally, acid catalyzed reactions are used to convert FFAs to esters, or soaps to esters as a pretreatment step for high FFA feedstocks. Residence times from 10 minutes to about 2 hours are reported.

Counter current acid esterification systems have been used for decades to convert pure streams of fatty acids into methyl esters at yields above $99 \%$. These systems tend to force yields to $100 \%$ and wash water out of the system at the same time because the feedstock and the sulfuric $\mathrm{acid} / \mathrm{methanol} \mathrm{mix}$ are moving in opposite directions. Acid esterification systems produce a byproduct of water. In batch systems, the water tends to accumulate in the vessel to the point where it can shut the reaction down prematurely. The sulfuric acid tends to migrate into the 
water, out of the methanol, rendering it unavailable for the reaction. All acid esterification systems need to have a water management strategy. Good water management can minimize the amount of methanol required for the reaction. Excess methanol (such as the 20:1 ratio) is generally necessary in batch reactors where water accumulates. Another approach is to approach the reaction in two stages: fresh methanol and sulfuric acid is reacted, removed, and replaced with more fresh reactant. Much of the water is removed in the first round and the fresh reactant in the second round drives the reaction closer to completion. Acid-catalyzed esterification is discussed in more detail in the chapter on Pretreatment of High FFA Feedstocks.

Lipase catalyzed reactions have the advantage reacting a room temperature without producing spent catalysts. The enzymes can be recycled for use again or immobilized onto a substrate. If immobilized, the substrate will require replacement when yields begin to decline. The enzyme reactions are highly specific. Because the alcohol can be inhibitory to some enzymes, a typical strategy is to feed the alcohol into the reactor in three steps of 1:1 mole ratio each. The reactions are very slow, with a three step sequence requiring from 4 to 40 hours, or more. The reaction conditions are modest, from 35 to $45^{\circ} \mathrm{C}$. Transesterification yields generally do not meet ASTM standards, but esterification yields can occur relatively quickly and yields are good. Excess free fatty acids can be removed as soaps in a later transesterification or caustic stripping step.

\section{Biodiesel Production Process Options}

\section{Batch Processing}

The simplest method for producing alcohol esters is to use a batch, stirred tank reactor. Alcohol to triglyceride ratios from 4:1 to 20:1 (mole:mole) have been reported, with a 6:1 ratio most common. The reactor may be sealed or equipped with a reflux condenser. The operating temperature is usually about $65^{\circ} \mathrm{C}$, although temperatures from $25^{\circ} \mathrm{C}$ to $85^{\circ} \mathrm{C}$ have been reported.

The most commonly used catalyst is sodium hydroxide, with potassium hydroxide also used. Typical catalyst loadings range from $0.3 \%$ to about $1.5 \%$.

Thorough mixing is necessary at the beginning of the reaction to bring the oil, catalyst and alcohol into intimate contact. Towards the end of the reaction, less mixing can help increase the extent of reaction by allowing the inhibitory product, glycerol, to phase separate from the esteroil phase. Completions of $85 \%$ to $94 \%$ are reported.

Some groups use a two-step reaction, with glycerol removal between steps, to increase the final reaction extent to $95+$ percent. Higher temperatures and higher alcohol:oil ratios also can enhance the percent completion. Typical reaction times range from 20 minutes to more than one hour.

Figure 14 shows a process flow diagram for a typical batch system. The oil is first charged to the system, followed by the catalyst and methanol. The system is agitated during the reaction time. Then agitation is stopped. In some processes, the reaction mixture is allowed to settle in the reactor to give an initial separation of the esters and glycerol. In other processes the reaction mixture is pumped into a settling vessel, or is separated using a centrifuge. 
The alcohol is removed from both the glycerol and ester stream using an evaporator or a flash unit. The esters are neutralized, washed gently using warm, slightly acid water to remove residual methanol and salts, and then dried. The finished biodiesel is then transferred to storage. The glycerol stream is neutralized and washed with soft water. The glycerol is than sent to the glycerol refining section.

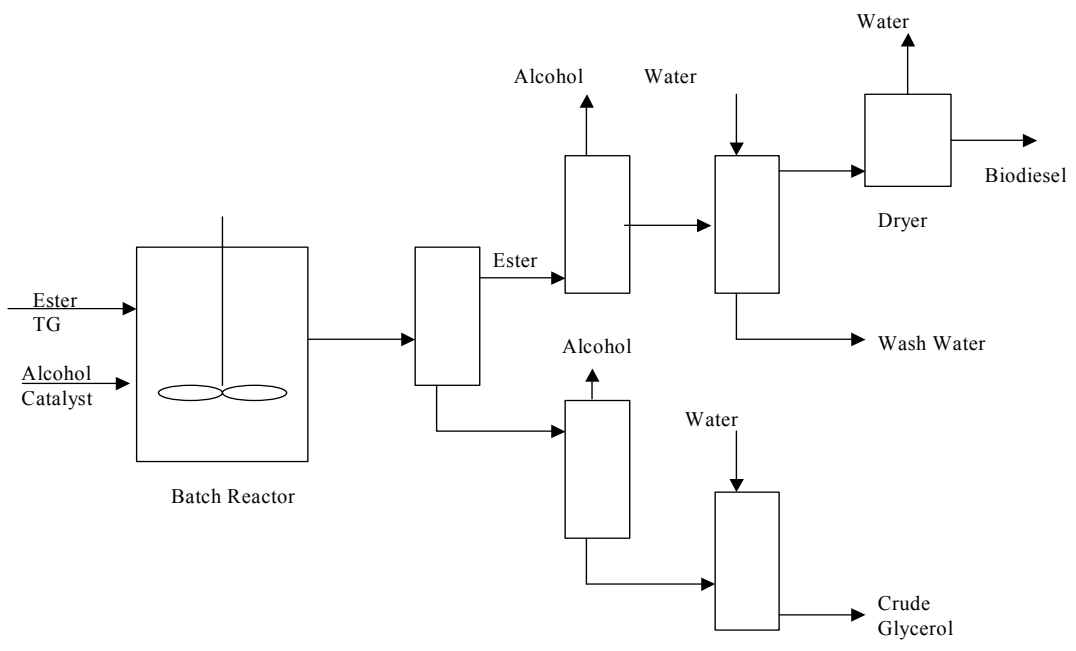

Figure 14. Batch Reaction Process

For yellow grease and animal fats, the system is slightly modified with the addition of an acid esterification vessel and storage for the acid catalyst. The feedstock is sometimes dried (down to $0.4 \%$ water) and filtered before loading the acid esterification tank. The sulfuric acid and methanol mixture is added and the system is agitated. Similar temperatures to transesterification are used and sometimes the system is pressurized or a cosolvent is added. Glycerol is not produced. If a two-step acid treatment is used, the stirring is suspended until the methanol phase separates and is removed. Fresh methanol and sulfuric acid is added and the stirring resumes. Once the conversion of the fatty acids to methyl esters has reached equilibrium, the methanol/water/acid mixture is removed by settling or with a centrifuge. The remaining mixture is neutralized or sent straight into transesterification where it will be neutralized using excess base catalysts. Any remaining free fatty acids will be converted into soaps in the transesterification stage. The transesterification batch stage processes as described above.

Continuous Process SystemsA popular variation of the batch process is the use of continuous stirred tank reactors (CSTRs) in series. The CSTRs can be varied in volume to allow for a longer residence time in CSTR 1 to achieve a greater extent of reaction. After the initial product glycerol is decanted, the reaction in CSTR 2 is rather rapid, with $98+$ completion not uncommon.

An essential element in the design of a CSTR is sufficient mixing input to ensure that the composition throughout the reactor is essentially constant. This has the effect of increasing the dispersion of the glycerol product in the ester phase. The result is that the time required for phase separation is extended. 
There are several processes that use intense mixing, either from pumps or motionless mixers, to initiate the esterification reaction. Instead of allowing time for the reaction in an agitated tank, the reactor is tubular. The reaction mixture moves through this type of reactor in a continuous plug, with little mixing in the axial direction. This type of reactor, called a plug-flow reactor (PFR), behaves as if it were a series of small CSTRs chained together.

The result is a continuous system that requires rather short residence times, as low as 6 to 10 minutes, for near completion of the reaction. The PFRs can be staged, as shown, to allow decanting of glycerol. Often this type of reactor is operated at an elevated temperature and pressure to increase reaction rate. A PFR system is shown in Figure 15.

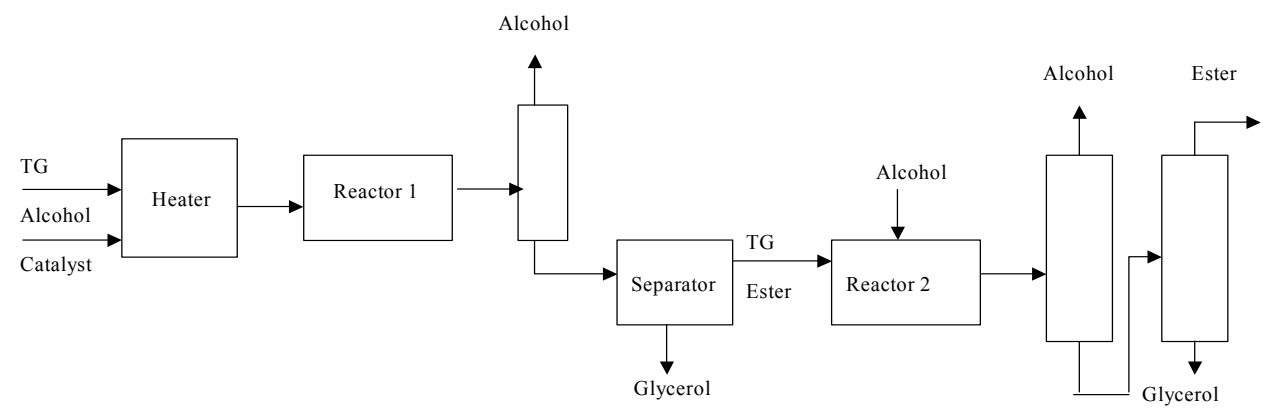

Figure 15. Plug Flow Reaction System

\section{High Free Fatty Acid Systems}

High free fatty acid feedstocks will react with the catalyst and form soaps if they are fed to a base catalyzed system. The maximum amount of free fatty acids acceptable in a base catalyzed system is less than 2 percent, and preferably less than 1 percent. Some approaches to using high free fatty acid feedstocks use this concept to "refine" the free fatty acids out of the feed for disposal or separate treatment in an acid esterification unit. The caustic is added to the feedstock and the resulting soaps are stripped out using a centrifuge. This is called caustic stripping.

Some triglycerides are lost with the soaps during caustic stripping. The soap mixture can be acidulated to recover the fatty acids and lost oils in a separate reaction tank. The refined oils are dried and sent to the transesterification unit for further processing. Rather than waste the free fatty acids removed in this manner, they can be transformed into methyl esters using an acid esterification process. As described earlier, acid catalyzed processes can be used for the direct esterification of free fatty acids in a high FFA feedstock. Less expensive feedstocks, such as tallow or yellow grease, are characteristically high in free fatty acids (FFA). The standard for tallow and yellow grease is $\leq 15$ percent FFA. Some lots may exceed this standard.

Direct acid esterification of a high free fatty acid feed requires water removal during the reaction, or the reaction will be quenched prematurely. Also, a high alcohol to FFA ratio required, usually 
between 20:1 and 40:1. Direct esterification may also require rather large amounts of the acid catalyst depending on the process used.

The esterification reaction of FFAs with methanol produces byproduct water that must be removed, but the resulting mixture of esters and triglyceride, can be used directly in a conventional base catalyzed system. The water can be removed by vaporization, settling, or centrifugation as a methanol-water mixture. Counter-current continuous-flow systems will wash out the water with the exiting stream of acidic methanol.

One approach to the acid catalyst system has been to use phosphoric acid as the initial catalyst, neutralize with an excess of $\mathrm{KOH}$ for the base step, and then neutralize with phosphoric acid upon completion. The insoluble potassium phosphate is recovered, washed, and dried for use as a fertilizer. Figure 16 shows a typical acid catalyzed, direct esterification process.

An alternative approach to utilization of high FFA feedstocks is to use a base catalyst to deliberately form soap from the FFAs. The soap is recovered, the oil dried, and then used in a conventional base catalyzed system.

This strategy can lead to a false sense of economy. If the soapstock is discarded, the effective price of the feedstock is increased in inverse proportion to the percentage of remaining oil. The soapstock can, however, be converted into esters by using an acid catalyzed reaction. The problem with this strategy is that the soapstock system contains a large amount of water that must be removed before the product esters can meet the biodiesel standard. The soapstock process is shown in Figure 17.

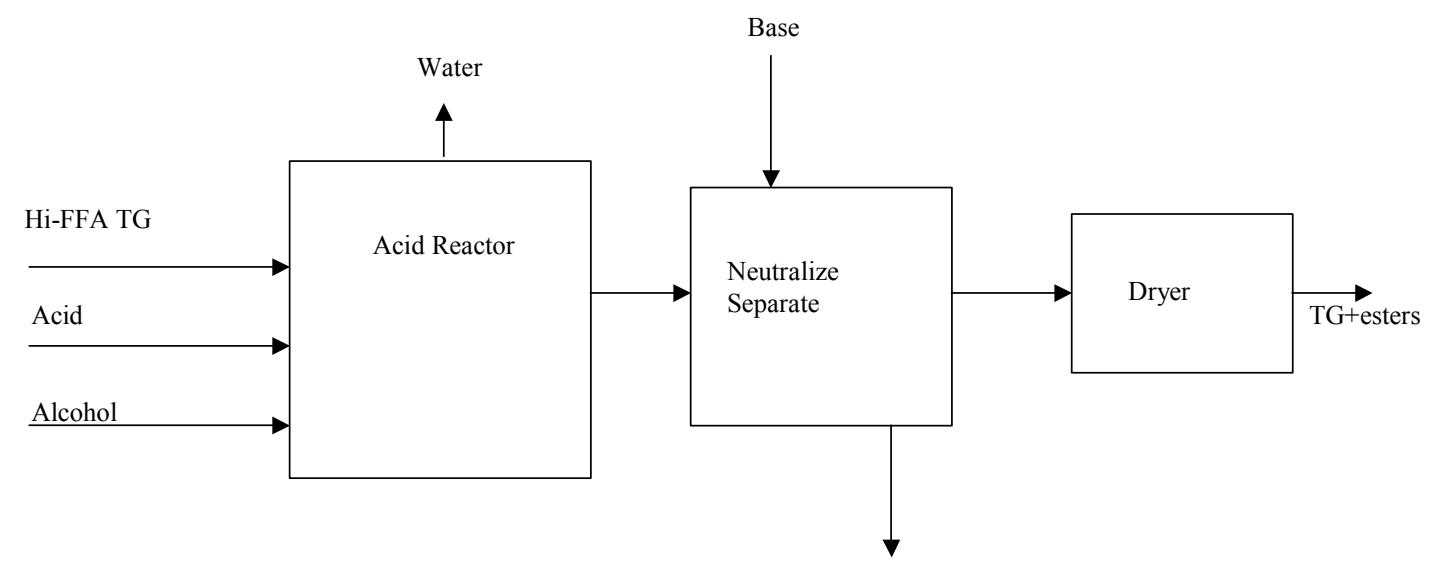

Figure 16. Acid Catalyzed Direct Esterification Process

An alternative procedure for processing high FFA feeds is to hydrolyze the feedstock into pure FFA and glycerine. Typically this is done in a counter current reactor using sulfuric/sulfonic acids and steam. The output is pure free fatty acids and glycerin. Any contaminates in the feedstock partition mostly into the glycerin and some may leave with the steam/water effluent. Some contaminants continue with the FFA and can be removed or left in, depending on the processes and product specifications. The pure FFA are then acid esterified in another counter current reactor to transform them into methyl esters. The methyl esters are then neutralized and 
dried. Yields can exceed 99\%. Equipment needs to be acid resistant but generally feedstock costs are extremely low.

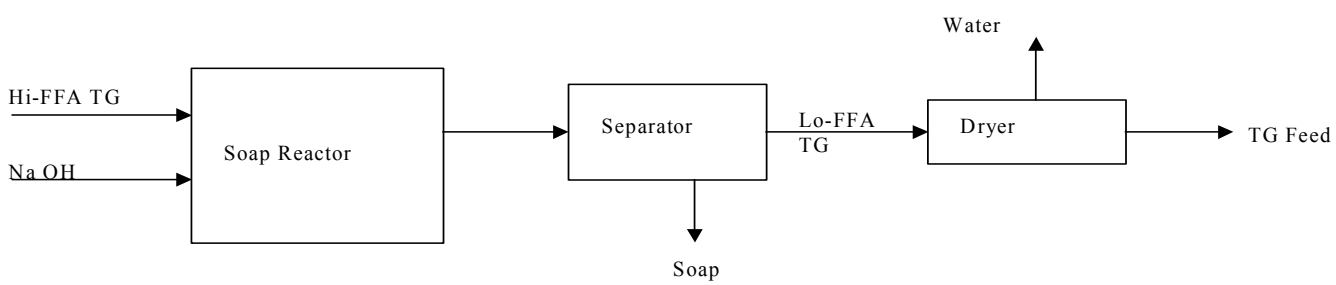

Figure 17. Preparation of Soapstock from a High FFA Feed

A variation of the base-catalyzed system that avoids the problem of high FFAs is the use of a fixed bed, insoluble base. An example of this system, using calcium carbonate as the catalyst, has been demonstrated at the bench-scale. This process is depicted in Figure 18.

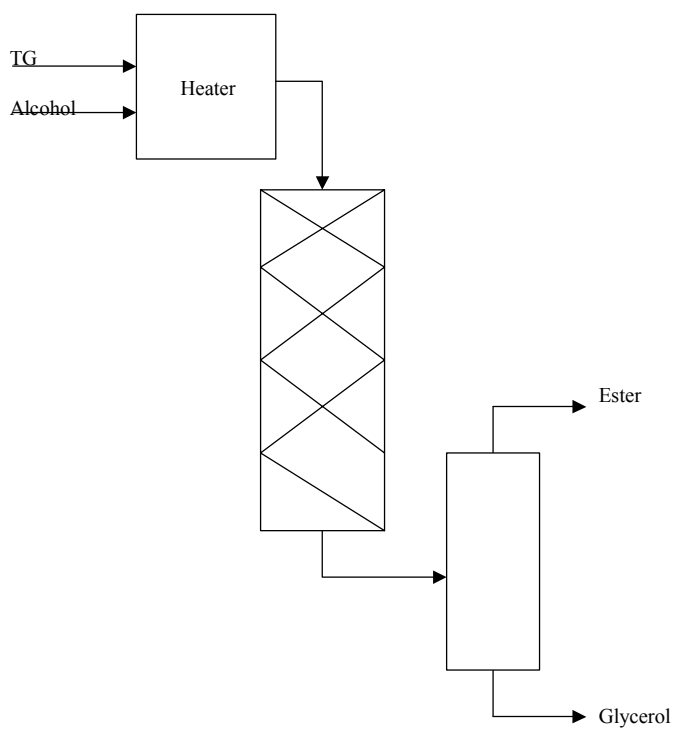

Figure 18. Fixed-Bed, Base Catalyzed Reactor System

\section{Non-Catalyzed Systems - Biox Process}

Cosolvent options are designed to overcome slow reaction time caused by the extremely low solubility of the alcohol in the TG phase. One approach that is nearing commercialization is the Biox Process. This process uses a co-solvent, tetrahydrofuran, to solubilize the methanol. The result is a fast reaction, on the order of 5 to 10 minutes, and no catalyst residues in either the ester or the glycerol phase. The THF co-solvent is chosen, in part, because it has a boiling point very close to that of methanol. After the reaction is complete, the excess methanol and the tetrahydrofuran co-solvent are recovered in a single step. This system requires a rather low operating temperature, $30^{\circ} \mathrm{C}$. Other cosolvents, such as MTBE, have been investigated. 
The ester-glycerol phase separation is clean and the final products are catalyst- and water-free. The equipment volume has to be larger for the same quantity of final product because of the additional volume of the co-solvent. The Biox Process is depicted in Figure 19. Cosolvents that are subject to the hazardous and/or air toxic EPA list for air pollutants require special "leak proof" equipment the entire sytem including methanol/cosolvent recovery and recycling. Fugitive emissions are tightly controled. The cosolvent must be completely removed from the glycerin as well as the biodiesel.

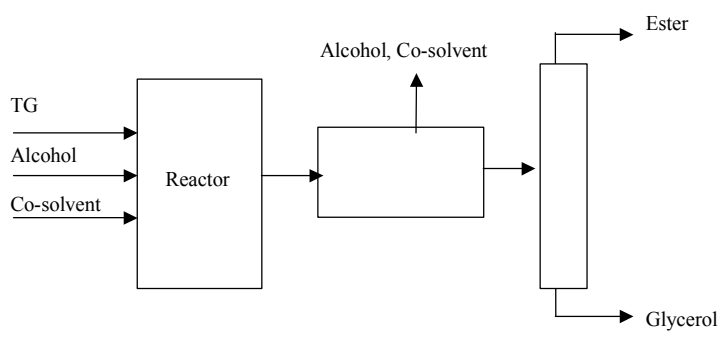

Figure 19. Biox Co-Solvent Process

\section{Non-Catalyzed Systems - Supercritical Process}

When a fluid or gas is subjected to temperatures and pressures in excess of its critical point, there are a number of unusual properties exhibited. There no longer is a distinct liquid and vapor phase, but a single, fluid phase present. Solvents containing a hydroxyl $(\mathrm{OH})$ group, such as water or primary alcohols, take on the properties of super-acids.

A non-catalytic approach is the use of a high (42:1) alcohol to oil ratio. Under supercritical conditions ( 350 to $400{ }^{\circ} \mathrm{C}$ and $>80$ atm or $1200 \mathrm{psi}$ ) the reaction is complete in about 4 minutes. Capital and operating costs can be more expensive, and energy consumption higher.

An intriguing example of this process has been demonstrated in Japan, where oils in a very large excess of methanol have been subjected to very high temperatures and pressures for a short period of time. The result is a very fast ( 3 to 5 minute) reaction to form esters and glycerol. The reaction must be quenched very rapidly so that the products do not decompose. The reactor used in the work to date is a $5 \mathrm{ml}$ cylinder that is dropped into a bath of molten metal, and then quenched in water.

Clearly, while the results are very interesting, the scale-up to a useful process may be quite difficult. Figure 20 depicts one conception of a configuration for a supercritical esterification process. 


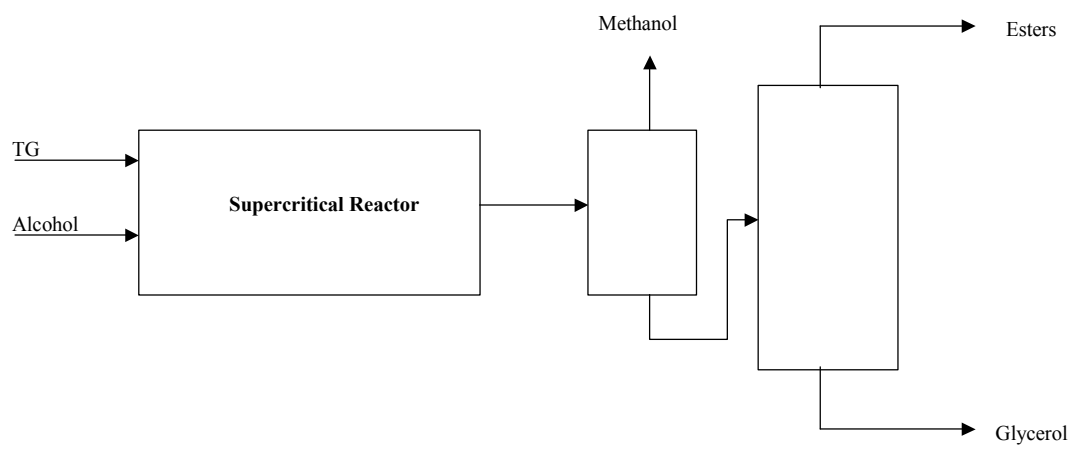

Figure 20. Supercritical esterification process

The refining of glycerol is discussed in a separate chapter, although it is a topic of great importance to the choice of biodiesel production process. Generally the quality of the glycerol produced and its value as a coproduct is an important economic variable. The typical glycerol produced by a biodiesel plant is $50 \%$ glycerol or less and contains water, salts, methanol, methyl esters, and unreacted glycerides, color and odor bodies, dimers, timers, and other minor compounds. This is commonly referred to as "biodiesel crude" and is generally worth less than 5 cents per pound. Removing the water and methanol and bringing the glycerol content up to $88 \%$ can generate a credit based on the value of crude glycerol. Salts, and particularly sulfur salts or potassium salts reduces the value of the crude glycerol produced.

\section{Summary}

There are multiple operating options available for making biodiesel. Many of these technologies can be combined under various conditions and feedstocks in an infinite number of ways. The technology choice is a function of desired capacity, feedstock type and quality, alcohol recovery, and catalyst recovery. The dominant factor in biodiesel production is the feedstock cost, with capital cost contributing only about $7 \%$ of the final product cost.

However, some reaction systems are capable of handling a variety of feedstocks and qualities, while others are not. Also, the different approaches to the esterification process result in quite different operating requirements, different water use requirements, and different operating modes.

In general, smaller capacity plants and variable feedstock quality suggest use of batch systems. Continuous systems generally lead the operation on a 24/7 basis, requiring larger capacities to justify larger staffing requirements and require a more uniform feedstock quality. 


\section{Laboratory - Exercise 1}

\section{Investigations of Biodiesel Production Pitfalls}

Equipment needed:

Four $500 \mathrm{ml}$ Erlenmeyer flasks containing $200 \mathrm{~g}$ of soybean oil

Four $125 \mathrm{ml}$ Erlenmeyer flasks, 3 containing $43 \mathrm{~g}$ of methanol, one containing $21.5 \mathrm{~g}$ of methanol

Four hot plate/stirrers

Four separatory funnels with ringstands

Aluminum foil (for capping flasks)

Small bottles containing preweighed sodium hydroxide, three with $2 \mathrm{~g}$, one with $10 \mathrm{~g}$.

Group 1: Too much water (How much harm can a little water do?)

- Starting with $200 \mathrm{~g}$ of soybean oil in a $500 \mathrm{ml}$ flask. Add $10 \mathrm{~g}$ of water.

- Add $2 \mathrm{~g}$ of sodium hydroxide pellets to $43 \mathrm{~g}$ of methanol and agitate until the pellets are dissolved.

- Add the methanol/sodium hydroxide solution to the soybean oil. Add a magnetic stirring bar and place on the hot plate. Heat to $60-65^{\circ} \mathrm{C}$ and agitate for 1 hour. Pour into a separatory funnel and allow to separate.

Does separation occur?

What happens when you add water?

Group 2: Too much catalyst (If a little bit is good, more must be better)

- Start with $200 \mathrm{~g}$ of soybean oil in a $500 \mathrm{ml}$ flask.

- Add $10 \mathrm{~g}$ of sodium hydroxide pellets to $43 \mathrm{~g}$ of methanol and agitate until the pellets are dissolved. Heating may be required.

- Add the methanol/sodium hydroxide solution to the soybean oil. Add a magnetic stirring bar and place on the hot plate. Heat to $60-65^{\circ} \mathrm{C}$ and agitate for 1 hour. Pour into a separatory funnel and allow to separate.

Does separation occur?

What happens when you add water?

Group 3: Not enough alcohol (Why should I use more than the reaction needs?)

- Start with $200 \mathrm{~g}$ of soybean oil in a $500 \mathrm{ml}$ flask.

- Add $2 \mathrm{~g}$ of sodium hydroxide pellets to $21.5 \mathrm{~g}$ of methanol and agitate until the pellets are dissolved. Heating may be required.

- Add the methanol/sodium hydroxide solution to the soybean oil. Add a magnetic stirring bar and place on the hot plate. Heat to $60-65^{\circ} \mathrm{C}$ and agitate for 1 hour. Pour into a separatory funnel and allow to separate.

Does separation occur?

What happens when you add water?

Group 4: High free fatty acid feedstock (Why treat this any differently than refined oil?)

- Start with $200 \mathrm{~g}$ of animal fat in a $500 \mathrm{ml}$ flask. 
- Add $2 \mathrm{~g}$ of sodium hydroxide pellets to $43 \mathrm{~g}$ of methanol and agitate until the pellets are dissolved.

- Add the methanol/sodium hydroxide solution to the animal fat. Add a magnetic stirring bar and place on the hot plate. Heat to $60-65^{\circ} \mathrm{C}$ and agitate for 1 hour. Pour into a separatory funnel and allow to separate.

Does separation occur?

What happens when you add water? 


\section{Basic Plant Equipment and Operation}

The intent of the basic plant equipment and operation section is to discuss the principles behind the primary plant equipment that would be used in a biodiesel production facility. Included will be discussion of reactors (both batch and continuous types), pumps, centrifuges, and distillation columns. Although there will be additional equipment in the plant such as settlers, storage tanks, etc., the four classes of equipment discussed here represent the heart of the process.

\section{Reactors}

The reactor is the only place in the process where chemical conversion occurs. Therefore, it dictates what chemical species must be handled downstream of the reactor. Reactors can be placed into two broad categories, batch reactors and continuous reactors. Two of reactors within the continuous reactor category are continuous stirred tank reactors (CSTRs) and plug flow reactors (PFRs). In the batch reactor, the reactants are charged into the reactor at the determined amount. The reactor is then closed and taken to the desired reaction conditions (temperature, pressure, and stir rates). The chemical composition within the reactor changes with time. Once the prescribed reaction time is complete, the chemical contents of the reactor are removed and sent for subsequent processing. In contrast, continuous reactors have a steady flow of reactants into the reactor and products out of the reactor. Once a continuous flow reactor reaches steadystate operation, the product composition leaving the reactor becomes constant. For CSTRs, the reactants are fed into a well-mixed reactor. The composition of the product stream is identical to the composition within the reactor. Hold-up time in a CSTR is given by a residence time distribution. For PFRs, the reactants are fed into one side of the reactor. The chemical composition changes as the material moves in plug flow through the reactor. The residence time is defined by the length of time required for molecules to travel through the reactor.

The most important considerations within a reactor are the extent of reaction of the reactants, which is known as conversion, and the selectivity of the reaction to the desired products, which is known as the selectivity. Key reactor variables that dictate conversion and selectivity are temperature, pressure, reaction time (residence time), and degree of mixing. In general, increasing the reaction temperature increases the reaction rate and, hence, the conversion for a given reaction time. However, if more than one reaction is taking place, the selectivity to the desired products can be impacted by changing the reaction temperature. In the transesterification reaction, the selectivity of the reaction is not negatively impacted by increasing temperature. Increasing temperature in the transesterification reaction does impact the operating pressure. The reaction is a liquid phase reaction, so the pressure in the reactor must be maintained at a level that keeps the methanol in the liquid phase. Therefore, as the reaction temperature is increased the pressure must also be increased. Another means to increase the conversion in a reactor is to increase the reaction time. For a given temperature, conversion will increase as the reaction time increases. Increasing the reaction time has the negative effect of decreasing the chemical throughput in a given plant or increasing the reactor size for a given chemical throughput. A final very important parameter in the reactor is the degree of mixing or mass transfer. For batch reactors and CSTRs the degree of mixing is directly related to the amount of energy introduced through the impeller. Increasing the speed of the impeller, which increases the energy input to the reactor, increases the degree of mixing, which improves the performance of the reactor. A 
threshold exists where additional mixing will not provide any performance enhancement. For PFRs, the degree of mixing is dictated by the design of the reactor and/or catalyst used within the reactor, since no mixing device is present in the reactor that can be independently manipulated.

Batch reactors have several positive features including good mixing characteristics and relative ease of handling homogeneous catalysts as used in the biodiesel transesterification reaction. As mentioned above, the reactant concentrations change with time in the reactor as does the product concentration. Therefore, the effect of reaction inhibition by reaction products such as experienced with glycerol can be minimized. However, batch reactors are generally not used in the production of large volume chemicals since it is most efficient to operate the subsequent separation and purification steps in a continuous mode. This batch to continuous mode can be overcome by using multiple reaction vessels sequenced to give effectively continuous operation or by employing a large "surge" vessel after the reactor.

CSTRs, which obviate the primary problem with batch reactors, also provide good mixing characteristics. However, the uniform concentration present in the reactor gives a lower reaction driving force and can also maximize reaction inhibition effects. PFRs or tubular reactors are the most common reactors in the chemical industry. Despite being continuous, the reactant concentration in these reactors changes with distance down the bed to maximize the driving force for the reaction, which is similar to a batch reactor. Achieving good mixing can be more problematic with PFRs and these type of reactors typically use solid catalysts.

\section{Pumps}

While the role of pumps in a chemical plant seems rather mundane, they play the key role in moving chemicals through the manufacturing plant. The most common type of pump in the chemical industry is a centrifugal pump. In many applications, the fluid shear introduced by a centrifugal pump is not a point of concern. However, the shear created by a centrifugal pump can create emulsion problems for the product stream from the biodiesel reactor. The amount of fluid shear imparted by the pump can be significantly reduced by using a positive displacement pump.

There are a number of different types of positive displacement pumps including gear pumps (external and internal) and lobe pumps. External gear pumps generally have two gears with equal number of teeth located on the outside of the gears, whereas, internal gear pumps have one larger gear with internal teeth and a smaller gear with external teeth. The liquid is "pumped" from the action of being carried between the gear teeth followed by displacement as the teeth mesh. In most external gear pumps, one gear drives the other. The internal gear pump has the advantage of fewer moving parts, but it cannot handle liquid streams containing solids or abrasives.

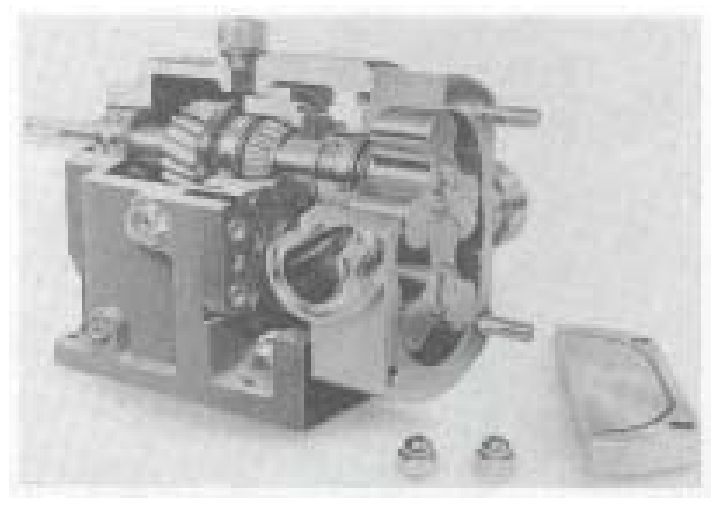

Lobe pumps impart less shear to the fluid being pumped than gear pumps. The lobes in the pump are designed for no contact, so timing gears are required for each lobe. Therefore, the lobe pump has more moving parts than gear pumps. Lobe pumps are best for handling "fragile" and high 
viscosity fluids at low pressures. As with gear pumps, lobe pumps work by carrying the fluid between lobes followed by displacement as the lobes come together. Since the lobes are designed for no contact, fairly high amounts of slip can occur if the fluid to be pumped has low viscosity.

Centrifugal pumps convert velocity into feet of head where the feet of head is independent of the density of the liquid being pumped. However, conversion of the feet of head to output pressure requires inclusion of the fluid density. For example, 231 feet of water in a column, i.e., 231 feet of head, exerts a pressure of $100 \mathrm{psig}$ at the base, whereas the same 231 feet of biodiesel with specific gravity of 0.88 would exert a pressure of 88 psig. Therefore, the discharge pressure of the pump when pumping water would be higher than with biodiesel.

The primary components of a centrifugal pump are (1) a shaft, (2) a coupling attaching the shaft to a motor, (3) bearings to support the shaft, (4) a seal around the shaft to prevent leakage, (5) an impeller, and (6) a volute, which converts the kinetic energy imparted by the impeller into feet of head. The role of the impeller is to increase the kinetic energy of the liquid. The liquid enters

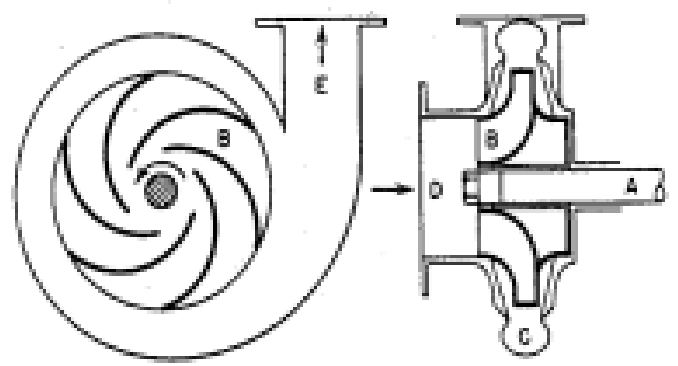
and leaves the impeller at the same pressure. As the liquid flows through the volute, the velocity is decreased with the energy transferred to pressure.

While leaking pump seals or impeller wear can be operational issues with pumps, the most common issue with pumps is the loss of suction pressure. Suction pressure is the pressure supplied to the suction side of the pump by the liquid. During loss of suction pressure, which is known as cavitation, the pump discharge pressure and the discharge flow rates become erratic and low. Cavitation is created by vaporization of liquid at the pump suction. This situation can be created be a plug in the suction piping or by starting the pump up too rapidly. In general, positive displacement pumps are more difficult to cavitate than centrifugal pumps. 


\section{Centrifuges}

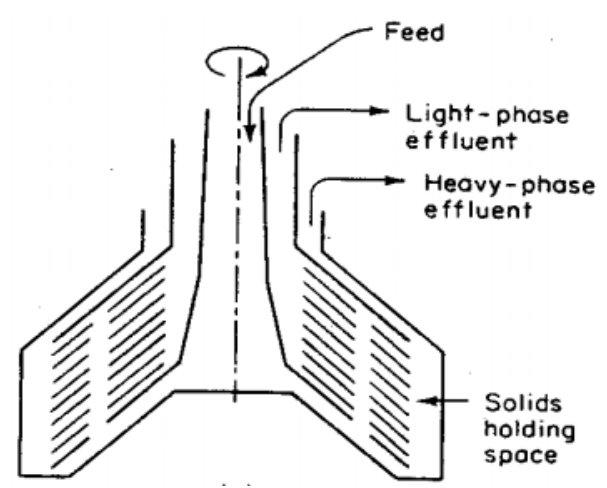

Centrifuges are most typically used to separate solids and liquids, but they can also be used to separate immiscible liquids of different densities. This type of separation can also be achieved using a settling tank. While a settling tank may be cheaper, a centrifuge can be used to increase the rate of separation relative to a settling tank. In a centrifuge the separation is accomplished by exposing the mixture to a centrifugal force. The more dense phase will be preferentially separated to the outer surface of the centrifuge. A centrifuge generally consists of (1) a bowl containing

the mixture, (2) a drive shaft and drive-shaft bearings, (3) a drive mechanism, and (4) a casing to segregate the separated products. Centrifuges are very amenable to continuous operation. A range of different centrifuge configurations are available with a common form being disk centrifuges.

The performance of a centrifuge depends on the specifications of the centrifuge as well as the characteristics of the mixture to be separated. While centrifuges are generally rated in terms of mixture handling capacity, the product quality specifications are the most important consideration. The choice of appropriate centrifuge type and size are predicated on the degree of separation needed in a specific system. The viscosity of the liquid has important implications on the choice of centrifuge size since higher viscosity fluids are more difficult to handle. If a sufficient density difference exists between immiscible fluids, centrifugation is a faster means of separation than settling.

\section{Distillation}

Another means of separating chemicals in a fluid mixture is by exploiting the differences in boiling points between the chemicals. If the boiling points are sufficiently different for the chemicals to be separated, such as with water and biodiesel, an evaporator or flash vaporizer can be used for the separation. In the evaporator, the liquid is heated to a temperature in which only the more volatile chemical species will vaporize. As such the vapor stream leaving the evaporator will be enriched in the more volatile species and the liquid stream from the evaporator will be enriched in the less volatile species. In an evaporator, the separation is accomplished by supplying heat while the mixture is held at a fixed pressure. In contrast, a flash vaporizer first heats the liquid, at an elevated pressure. Then, the heated liquid is sent through a flash valve that decreases the pressure. The decrease in pressure causes the more volatile portion of the liquid mixture to vaporize.

An important separation device for miscible fluids with similar boiling points (e.g., methanol and water) is the distillation column. Separation in a distillation column is predicated on the difference in volatilities (boiling points) between chemicals in a liquid mixture. In a distillation column the concentrations of the more volatile species are enriched above the feed point and the less volatile species are enriched below the feed point. The vaporization in the column is driven by heat supplied in the reboiler, which is subsequently removed in the overhead condenser. The 
temperature in the distillation column is the highest at the bottom and decreases moving up the column. Distillation columns can use either trays or packing.

The degree of separation that can be achieved in a distillation column is dictated by the relative volatilities of the chemicals to be separated, the number of trays or the height of the packing, and the reflux ratio. Chemicals with very different boiling points are easier to separate than those with similar boiling points. Increasing the number of trays or the height of packing can increase the amount of separation. Reflux ratio is the amount of condensed overhead vapor that is fed back into the top of the column. Increasing the reflux ratio increases the degree of separation. Increasing the number of trays or height of packing increases the column height and increasing the reflux ratio increases the column diameter. Either of these increases in column dimensions increases the capital cost of the column.

Trayed distillation columns are the most common. A tray is where contacting occurs between the vapor stream and the liquid stream. The liquid enters the feed tray through the downcomer from the above tray, flows across the tray where is interacts with the vapor, spills over a weir, and, finally, flows through the downcomer to the next tray. The vapor flows up through holes in the tray. It is important to have appropriate flow rates of the liquid and vapor to get optimal column operation. If the liquid flow rates become too high, poor contacting occurs with the vapor and in the extreme flooding of the column can occur. The onset of flooding in a column is easy to observe since the column pressure drop will increase markedly when flooding begins. If the vapor flow rate is too high, liquid can be entrained and carried over to the above tray leading to poorer separation. In contrast, too low of a vapor rate can lead to weeping of the liquid through the tray, which diminishes the separation. The liquid and vapor flow rates are dictated by the operation of the reboiler and the condenser, which means they are controlled by the energy input and removal.

The materials of construction is an important consideration for the reactor and storage tanks. The demands on the materials used for the storage tanks downstream of the reactor are lower since these tanks contain nearly $\mathrm{pH}$ neutral chemicals. The materials of construction for the storage tanks are discussed in the Transportation and Storage section. In contrast, the materials of construction required in the reactor must withstand basic conditions for the transesterification reaction or acidic conditions if an esterification approach is used to convert free fatty acids. For the base-catalyzed transesterification reaction, stainless steel is the preferred material for the reactor. However, stainless steel will not be good choice for use in the acid-catalyzed esterification reaction because stainless steel is subject to attack by acids. Under these conditions, an acid resistant material such as Hastelloy should be used for the reaction vessel. 


\section{Chemical Plant Controls}

To produce quality products from a biodiesel production plant it is necessary to be able to control the production process. While a tremendously important control variable is the properties of the feedstock to the process, this section will only discuss the control of chemical plants within the unit operation of the processes, such as those discussed in the previous section. Two general types of processes can be used in the production of biodiesel, either batch or continuous. For batch processes the process control variables will be temperature, pressure, and levels.

Temperature and pressure control are critical within the reaction and separation sections of the plants. Level control dictates charging to the reactor, operation of separation devices, and product storage. For continuous processes the process control variables include temperature, pressure, level, and flow rate.

It is important to note that process variables, which are monitored and controlled, are not the same as the product specifications. Specifications are properties that are required of the final product such as those given in ASTM D 6751 for biodiesel. Control variables are those process variables that are monitored and adjusted to meet the desired specifications. As such, specifications are generally measured off-line in an analytical laboratory, while the process variables are process conditions that are measured in real time. The process variables are related to the specifications through some type of process model. The process model will typically be provided by the process licensor with subsequent refinement from plant operation experience. Some manufacturing plants will develop their own statistical or empirical models. It should be noted that the process models are typically feedstock dependent.

In discussing process variable measurement instruments, the performance of the instruments can be characterized by their accuracy and/or precision. Accuracy is the ability of an instrument to measure the correct or "true" value of a process variable. In contrast, precision is the ability of an instrument to reproduce a process variable value within a certain interval. As such, it is possible to have either "accurate and imprecise" or "inaccurate and precise" instruments. For day-to-day operation the ability of a measurement instrument to be precise is generally more important than the need to be accurate. However, when the measurement instrument needs to be replaced, accuracy becomes an important issue.

When considering an instrumental control system for a chemical plant there are a number of factors that should be considered; cost, precision, reliability, and operator interface. It is desirable to have the lowest cost monitoring system that will allow appropriate control of the process. Precision is important to assure that there is reproducibility of measurements. Reliability insures that the system is not prone to failures. Finally, it is desirable to have a monitoring and control system that provides an easy interface with the plant operators. 


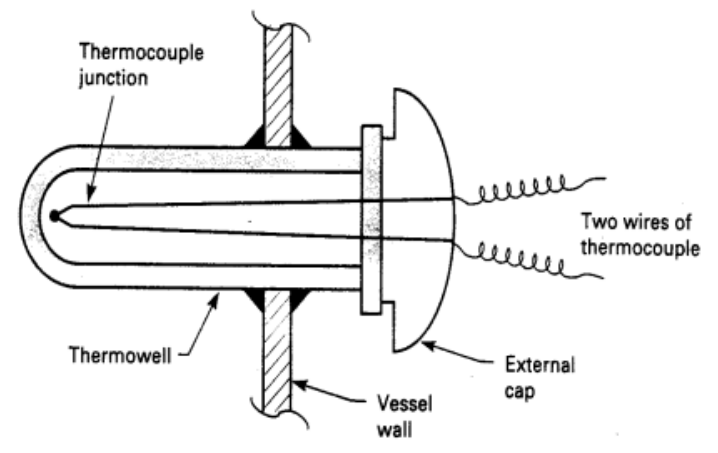

The most common process variable monitored in a chemical plant is the temperature. The temperature at a specific point is generally measured using thermocouples, which are electrical devices. Thermocouples are made of two different metallic wires. These wires are connected at a junction. An electrical current is established when the junction is heated. The electrical current is proportional to the temperature at the junction. For this reason, the thermocouple must be calibrated to correlate

temperature to current. Thermocouples are categorized by the metals used in their junctions, with different metal junction combinations being more appropriate for different temperature ranges. Individual thermocouples are typically not calibrated by the supplier. Instead, manufacturers will typically test a representative portion of the thermocouples they produce. Due to the manufacturing procedure, thermocouples are usually precise (or completely don't work) but can be inaccurate. The thermocouple is typically enclosed within a thermowell. The thermocouple must be fully inserted into the thermowell with the thermowell extended appropriately into the process to obtain a correct reading. If the temperature of a process liquid is being measured it is generally adequate for the thermowell to extend about 2-3 inches into the liquid. Due to inferior heat transfer, the thermowell should extend about 6 inches into a process vapor.

Thermocouples are typically used in processes for both monitoring and controlling the process. When a thermocouple is used as part of a process control loop, the electrical current output from the thermocouple is compared with the current expected for the set point temperature. Deviation of the actual temperature from the set point temperature will cause a change in the output to a heater or cooler that is included in the control loop.

Pressure values can be measured using several different types of devices such as liquid column, elastic element, and electrical sensing. The most common form of a liquid column device is a manometer in which the liquid height can be observed visually and correlated to the pressure through the density of the liquid. Liquid column devices are rarely used in chemical processes. Elastic element devices measure pressure by determining deformation of an elastic material. This elastic material is usually metallic. One type of elastic element device uses bellows elements. The displacement of the bellows can be correlated to the pressure value. The most common pressure measurement instruments used in chemical processes are electrical sensing devices, which are known as strain gauges. Strain gauges are predicated on the fact that the electrical resistance of conducting solids changes with length and diameter of the solid. The dimensional changes of the solid induced by a pressure causes an increase in the electrical resistance of the conducting solid. An elastic element or electrical sensing device used to measure pressure is called a pressure transducer. The pressure tap for a

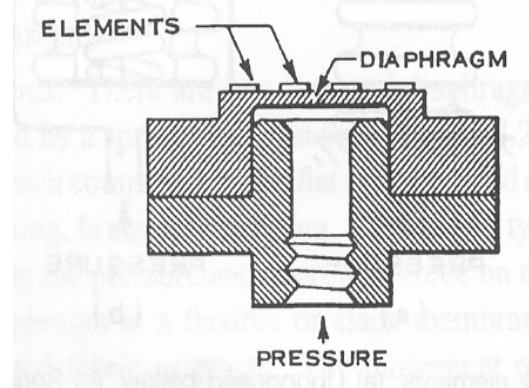
transducer should be located in the vapor phase of a process. A transducer located in the liquid phase will detect a different pressure at different depths in the liquid. 
To use a pressure transducer for process control or in continuous monitoring, the transducer must produce an electrical signal. For the elastic element device, the direct pressure reading due to displacement must somehow be converted to an electrical signal. An advantage of the electrical sensing approach is that these devices directly provide an electrical signal. As with thermocouples, pressure transducers can be used for process control by comparing the measured pressure to a desired set point pressure.

Level measurement can be used to yield mass balance information across the process or loading

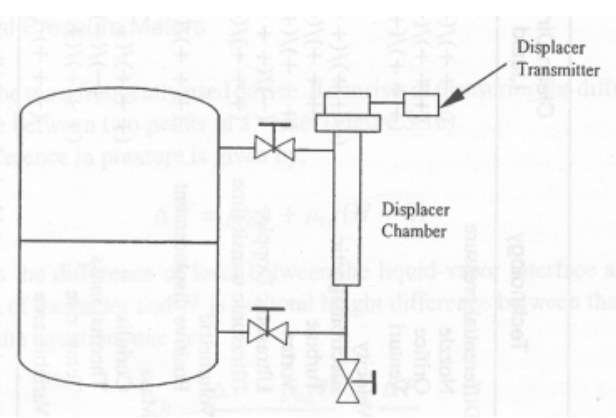
in a batch reactor. Historically, level measurement devices have typically been displacer-based meters or differential pressure meters. The displacer-based meter is based on buoyancy. The displacer is immersed in a displacer chamber that is located as a side chamber. The displacer is restrained by an elastic element whose motion is proportional to the buoyant force, so the level can be determined by the vertical location of the displacer. Differential pressure meters, which are the most common level indicating devices, measure the difference in pressure between two pressure taps in a vessel. It is important to note that both of these level measurement indicators are liquid density dependent, so that changes in liquid densities can affect their readings. This effect can be particularly important if several liquid phases are present in the device. For example, the height of liquid in a gauge glass connected to a vessel is not a direct measurement of the liquid level! Recently, level measurement devices have

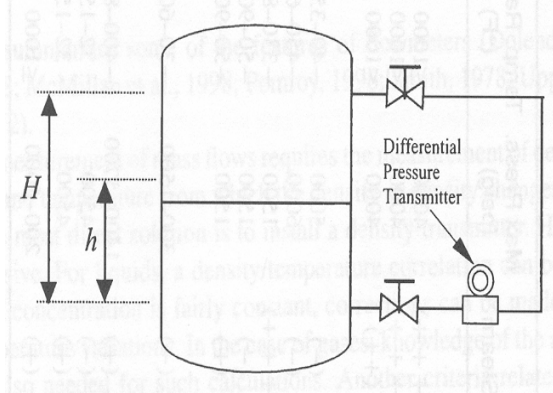
been developed that use signal reflectance from an ultrasonic transducer or radio frequency, which measures the change in impedance between two capacitor electrodes.

In addition to density, there are a number of factors that can influence the reliability of a level measurement. Plugged taps can occur without causing apparent discrepancies in the level measurement device. Excursions to high liquid levels can impact the reliability of the level measurement as can the presence of foam in the vessel.

The measurement and control of level in a vessel generally requires two taps into the vessel. It is important that these taps are properly valved. Since the level indicator can be used to monitor the amount of material in a vessel, it can be used to track chemical inventory within the process as well as to control the charging of chemicals into a batch process. Level measurements can be converted to electrical signals that can be used for control. The most common control loop that includes level measurement is with operation of pumps.

The final process variable that will be discussed is flow rate. Flow rate measurement will generally only be needed if a continuous flow process is used. There are a number of methods for measuring flow rates, but the most commonly used approach in chemical processes are 
differential pressure flowmeters and positive displacement flowmeters. Flowmeters can be used in monitoring and control. The most common control loop that includes flow rate is with operation of pumps.

Differential flowmeters measure the difference in pressure between the two sides of a restriction in a confined stream. These flowmeters are based on restrictions imposed by a venturi tube, an orifice plate or a flow nozzle with the orifice plate meter being the most common. The orifice plate hole should be appropriately sized for the flow rate range of interest. If the hole is too large the resulting pressure change and, therefore, accuracy of the flow measurement will be inaccurate. In contrast, if the hole is too small, the flow rate measurement is at the cost of too high of a pressure drop. While orifice plate meters are typically used for flow rate measurement and subsequent control, it should be noted that the meters do have some potential problems. First, this type of flowmeter is highly dependent on the density and viscosity of the liquid. Orifice plates can erode or have blockage, which will lead to inaccurate measurement. The pressure taps can become plugged leading to erroneous flow values.

Positive displacement flowmeters measure flow rates using the fluid to displace a measuring

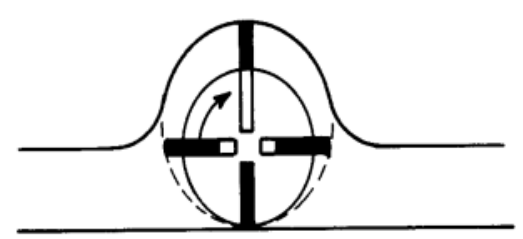
device. Most common are rotary displacement flowmeters. In these meters, flow rate is measured by the amount of rotation created by flow of the fluid past a rotary device. An example of a rotary flowmeter is a vane-liquid flowmeter in which a set of vanes mounted on a rotor with opposing pairs rotated in a cylindrical chamber.

The overall monitor and control system for a process can be achieved by individual monitors and controllers located directly at the process. However, it is most common in the chemical processes to have a centralized system for monitoring and controlling. This can be readily accommodated for most processes by a PC based system. A number of different software options are readily available for these types of systems. 


\section{Pretreatment of High Free Fatty Acid Feedstocks}

Many low cost feedstocks are available for biodiesel production. Unfortunately, many of these feedstocks contain large amounts of free fatty acids (FFAs). As discussed elsewhere, these free fatty acids will react with alkali catalysts to produce soaps that inhibit the reaction.

The following ranges of FFA are commonly found in biodiesel feedstocks:

Refined vegetable oils $\quad<0.05 \%$

Crude vegetable oil $\quad 0.3-0.7 \%$

Restaurant waste grease $\quad 2-7 \%$

Animal fat $\quad 5-30 \%$

Trap grease $\quad 40-100 \%$

Generally, when the FFA level is less than $1 \%$, and certainly if it is less than $0.5 \%$, the FFAs can be ignored. Common catalyst amounts are:

Sodium hydroxide: $1 \%$ of triglyceride weight

Potassium hydroxide: $1 \%$ of triglyceride weight

Sodium methoxide: $\quad 0.25 \%$ of triglycerides weight

Soaps may allow emulsification that causes the separation of the glycerol and ester phases to be less sharp. Soap formation also produces water that can hydrolyze the triglycerides and contribute to the formation of more soap. Further, catalyst that has been converted to soap is no longer available to accelerate the reaction.

When FFA levels are above $1 \%$, it is possible to add extra alkali catalyst. This allows a portion of the catalyst to be devoted to neutralizing the FFAs by forming soap, while still leaving enough to act as the reaction catalyst.

Since it takes one mole of catalyst to neutralize one mole of FFA, the amounts of additional catalyst can be calculated by the following formulas:

Sodium hydroxide: $[\%$ FFA $](0.144)+1 \%$

Potassium hydroxide: [\%FFA] $(0.197) / 0.86+1 \%$

Sodium methoxide: $[\% \mathrm{FFA}](0.190)+0.25 \%$

For example, when adding sodium methoxide to a feedstock with $1.5 \%$ FFA, the amount of catalyst would be:

$$
(1.5)(0.190)+0.25 \%=0.54 \% \text { of the triglycerides weight }
$$

Note that a factor of 0.86 has been included with the potassium hydroxide calculation to reflect that reagent grade $\mathrm{KOH}$ is only $86 \%$ pure. If other grades of catalyst are used, this factor should be adjusted to their actual purity. 
This approach to neutralizing the FFAs will sometimes work with FFA levels as high as $5-6 \%$. The actual limit can depend on whether other types of emulsifiers are present. It is especially important to make sure that the feedstock contains no water. 2-3\% FFA may be the limit if traces of water are present.

For feedstocks with higher amounts of FFA, the addition of extra catalyst may create more problems than it solves. The large amount of soap created can gel. It can also prevent the separation of the glycerol from the ester. Moreover, this technique converts the FFAs to a waste product when they could be converted to biodiesel.

When working with feedstocks that contain 5-30\% FFA or even higher, it is important to convert the FFAs to biodiesel or the process yield will be low. There are at least four techniques for converting the FFAs to biodiesel:

1. Enzymatic methods: These methods require expensive enzymes but seem to be less affected by water. At the present time, no one is using these methods on a commercial scale.

2. Glycerolysis: This technique involves adding glycerol to the feedstock and heating it to high temperature $\left(200^{\circ} \mathrm{C}\right)$, usually with a catalyst such as zinc chloride. The glycerol reacts with the FFAs to form mono- and diglycerides. Figure 21 shows the rate of decrease of the fatty acid level in a batch of animal fat. This technique produces a low FFA feed that can be processed using traditional alkali-catalyzed techniques.

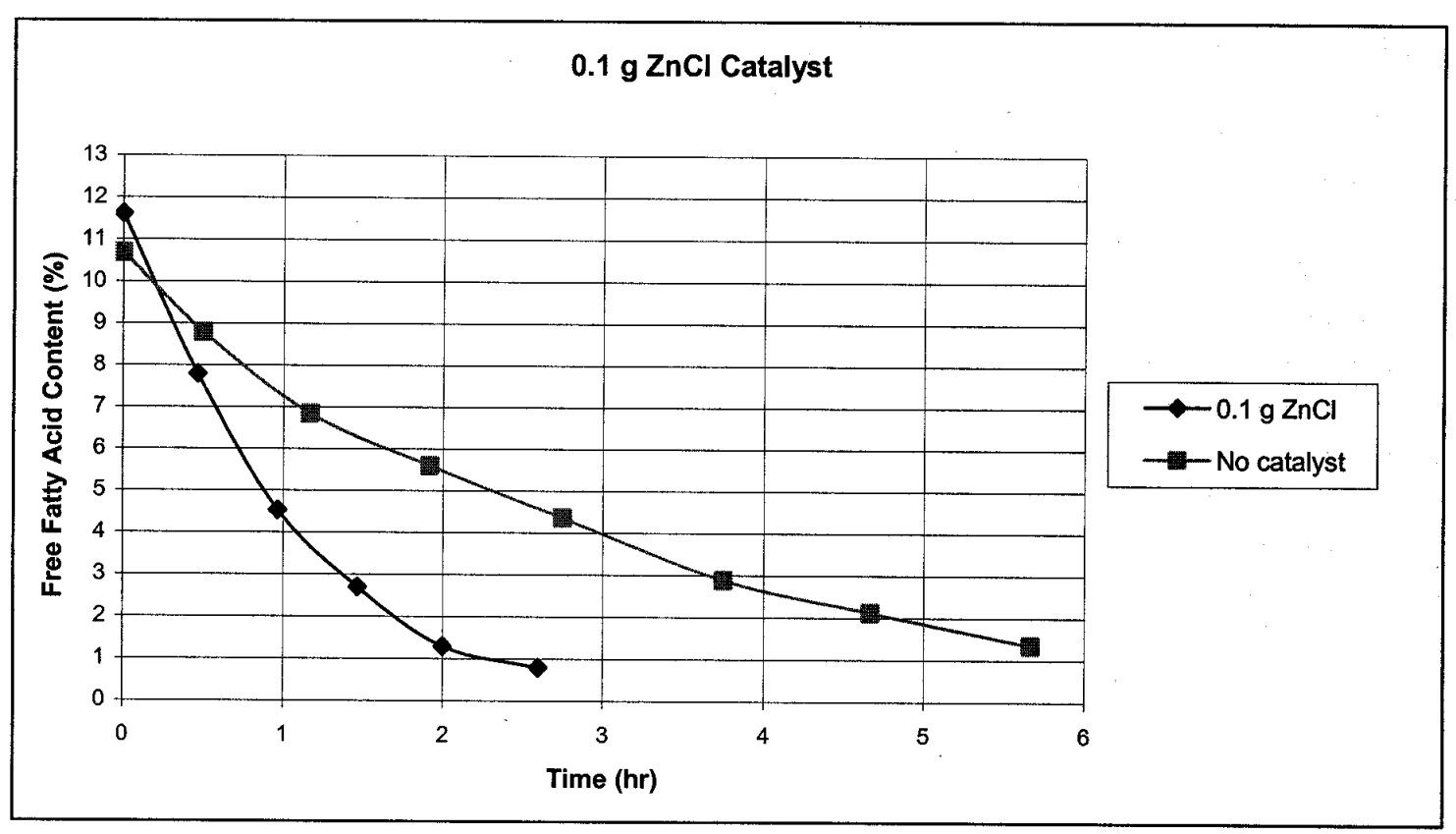

Figure 21. Reduction of Fatty Acids by Glycerolysis

(100 g animal fat, $13 \mathrm{~g}$ glycerol, $0.1 \mathrm{~g} \mathrm{ZnCl}, 200^{\circ} \mathrm{C}, 11 \mathrm{psi}$ vacuum) 
The drawback of glycerolysis is the high temperature and that the reaction is relatively slow. An advantage is that no methanol is added during the pretreatment so that as water is formed by the reaction

$$
\text { FFA }+ \text { glycerol } \rightarrow \text { monoglyceride }+ \text { water }
$$

The water immediately vaporizes and can be vented from the mixture.

3. Acid Catalysis: This technique uses a strong acid such as sulfuric acid to catalyze the esterification of the FFAs and the transesterification of the triglycerides. The reaction does not produce soaps because no alkali metals are present. The esterification reaction of the FFAs to alcohol esters is relatively fast, proceeding substantially to completion in one hour at $60^{\circ} \mathrm{C}$. However, the transesterification of the triglycerides is very slow, taking several days to complete. Heating to $130^{\circ} \mathrm{C}$ can greatly accelerate the reaction but reaction times will still be $30-45$ minutes. Another problem with acid catalysis is that the water production from the following reaction

$$
\text { FFA }+ \text { methanol } \rightarrow \text { methyl ester }+ \text { water }
$$

stays in the reaction mixture and ultimately stops the reaction, usually well before reaching completion.

4. Acid catalysis followed by alkali catalysis: This approach solves the reaction rate problem by using each technique to accomplish the process for which it is best suited. Since acid catalysis is relatively fast for converting the FFAs to methyl esters, it is used as a pretreatment for the high FFA feedstocks. Then, when the FFA level has been reduced to $0.5 \%$, or lower, an alkali catalyst is added to convert the triglycerides to methyl esters. This process can convert high free fatty acid feedstocks quickly and effectively. Water formation is still a problem during the pretreatment phase. One approach is to simply add so much excess methanol during the pretreatment that the water produced is diluted to the level where it does not limit the reaction. Molar ratios of alcohol to FFA as high as 40:1 may be needed. The disadvantage of this approach is that more energy is required to recover the excess methanol. Another approach would be to let the acid-catalyzed esterification proceed as far as it will go until it is stopped by water formation. Then, boil off the alcohol and water. If the FFA level is still too high, then additional methanol and, if necessary, acid catalyst can be added to continue the reaction. This process can be continued for multiple steps and will potentially use less methanol than the previous approach. Again, the disadvantage is the large amount of energy required by the distillation process.

A less energy intensive approach is to let the acid-catalyzed reaction mixture settle. After a few hours, a methanol-water mixture will rise to the top and can be removed. Then, additional methanol and acid can be added to continue the reaction. [Patent pending, Earl Hammond, ISU]. It is also possible to use fluids such as glycerol and ethylene glycol to wash the water from the mixture. 


\section{Procedure for High FFA Feedstocks}

1. Measure FFA level

2. Add $2.25 \mathrm{~g}$ methanol and $0.05 \mathrm{~g}$ sulfuric acid for each gram of free fatty acid in the oil or fat. Sulfuric acid and methanol should be mixed first and then added slowly to the oil.

3. Agitate for one hour at $60-65^{\circ} \mathrm{C}$.

4. Let mixture settle. Methanol-water mixture will rise to the top. Decant the methanol, water, and sulfuric acid layer.

5. Take bottom fraction and measure new FFA level.

6. If FFA is $>0.5 \%$, return to step 2 with new FFA level. If FFA is $<0.5 \%$, proceed to step 7.

7. Add an amount of methanol equal to $0.217 \mathrm{x}$ [grams of unreacted triglycerides] and an amount of sodium methoxide equal to [0.25 + (\%FFA) 0.190$] / 100 \times$ [grams of unreacted triglycerides]. Mix the sodium methoxide with the methanol and then add to the oil. This corresponds to a 6:1 molar ratio of methanol to oil for the unreacted triglycerides. It ignores any methanol that may have carried over from the pretreatment.

8. Agitate for 1 hour at $60^{\circ} \mathrm{C}$.

Example: $100 \mathrm{~g}$ of $12 \%$ FFA animal fat

Pretreatment: $2.25 \times 12 \mathrm{~g}=27.0 \mathrm{~g}$ methanol

$$
0.05 \times 12 \mathrm{~g}=0.6 \mathrm{~g} \mathrm{H}_{2} \mathrm{SO}_{4} \text { (sulfuric acid) }
$$

Mix acid with methanol. Then add mixture to fat. Agitate for one hour at $60^{\circ} \mathrm{C}$. Let settle and separate bottom phase. Acid value should decrease substantially, to at least 5-6 $\mathrm{mg} \mathrm{KOH} / \mathrm{g}$. There fore, $\mathrm{FFA}=2.5 \%$

Second step of pretreatment:

$$
\begin{aligned}
& 2.25 \times 2.5 \mathrm{~g}=5.6 \mathrm{~g} \text { of methanol } \\
& 0.05 \times 2.5 \mathrm{~g}=0.13 \mathrm{~g} \mathrm{H}_{2} \mathrm{SO}_{4}
\end{aligned}
$$

Mix with oil, agitate at $60^{\circ} \mathrm{C}$ for 1 hour. FFA should be $<0.5 \%$. Removal of upper phase is usually optional at this point.

Then add $\quad 0.217 \times(88)=19.1 \mathrm{~g}$ methanol

$$
[0.25+(0.5)(0.190)] / 100 \times 88=0.30 \mathrm{~g} \text { sodium methoxide }
$$

Agitate at $60^{\circ} \mathrm{C}$ for one hour. If glycerol and ester do not separate, add $50 \mathrm{~g}$ of warm distilled water to encourage separation. Wash 3-4 times. 


\section{Patent Discussion}

Although transesterification is an old and well-known process in organic chemistry, much of the modern chemical techniques were described in patents issued to chemists for DuPont and Colgate-Palmolove-Peet during and immediately after World War II. A careful examination of these patents shows that most of the techniques used since that time were anticipated and covered by these patents.

Prior to the 1940 s, most soap was made following a traditional process of saponifying fats through the addition of sodium or potassium hydroxide and water. The glycerol freed by this process usually remained with the soap and provided some moisturizing benefit. Techniques were available to separate the glycerol but they provided a water-contaminated crude product and were not suitable for use with potassium soaps. Because of the high demand for glycerol to produce explosives for the war effort, there was a strong motivation to remove the glycerol during soap production and provide a high-quality, dry product. George Bradshaw of E.I duPont received a patent $(\# 2,271,619)$ for a process that added about 1.6 times the theoretical amount of an alcohol, such as methanol, which contained 0.1 to $0.5 \%$ sodium or potassium hydroxide, to an oil or fat. When performed at $80^{\circ} \mathrm{C}$, this process provided $98 \%$ conversion to a high-quality, water-free glycerol and alcohol esters. The esters were then saponified with additional caustic soda to produce soap and the alcohol was recovered by heating. The residual glycerol and methanol in the alcohol esters could be removed by two or three washes with a small amount of water.

Bradshaw recognized that this process could be conducted in stages:

"... the amount [of alcohol] can be reduced substantially by working in steps.

For instance, if the theoretical quantity of methyl alcohol is used, the conversion will be about 80 per cent. The glycerine can then be removed and more alcohol and catalyst added. If the alcohol is added in three steps the amount required can be reduced, for instance, from one and six-tenths times theory to one and two-tenths times theory."

In his second patent $(\# 2,360,844)$ Bradshaw says that with three or four steps the amount of alcohol can be reduced to 1.1 times theory.

Bradshaw noted that other alcohols beside methanol could be used for the process including ethanol, propanol, isopropanol, the butanols, and the pentanols. Bradshaw further describes how fats containing small amounts of water and free fatty acids could still be processed with this technique by adding additional catalyst. He mentions that a drawback of this approach is that soap will be formed that can inhibit the separation of the glycerol. He suggests acidification of the reaction mixture to split the soaps back to free fatty acids and salts to facilitate the separation. His suggestions for acceptable acids include formic, acetic, propionic, hydrochloric, sulfuric, sulfamic, and phosphoric.

Bradshaw's work was followed by a extensive set of patents from researchers at the ColgatePalmolive-Peet company. These patents describe the same essential process as Bradshaw but add 
special features to improve the quality or quantity of the product. Each of these patents will be discussed with emphasis given to the unique contributions they make to the art.

H.D. Allen described modifications to Bradshaw's process that were intended to make it easier to implement as a continuous flow process. He raised the temperature of the reaction to the range $80^{\circ} \mathrm{C}$ to $160^{\circ} \mathrm{C}$ under enough pressure to keep the alcohol in the liquid phase. Allen noted that either acid or alkali catalysts could be used and that acid catalysts were better suited to feedstocks with high free fatty acid levels since the free fatty acids would be esterified during the process. At the higher temperatures, the reaction times were shortened to 3 to 4 minutes at $135^{\circ} \mathrm{C}$ with an alkali catalyst and 45 minutes with an acid catalyst. Allen's proposed system for transesterification is shown in Figure 1 and includes a reaction coil (plug flow reactor) to provide the time needed to complete the reaction.

Although previous researchers had noted the problem with using alkali catalysts with high free fatty acid feedstocks, and some had commented on the suitability of acid catalysts for this purpose, G.I. Keim appears to have been the first to propose a sequence of an acid catalyzed reaction to convert the free fatty acids to esters followed by an alkali catalyzed step to convert the triglycerides. Keim notes that the alkali catalysts are 10 to 50 times faster with triglycerides than the acid catalysts. However, the alkali reacts with the free fatty acids to form soap, which causes gelling problems and difficulty separating the glycerol. By starting with the acid to convert the FFAs to esters until the FFA level is at a tolerable amount for the alkali catalyst, then switching to the alkali catalyst, the reaction time is kept short without undesirable soap formation. Keim recognizes that the conversion of the FFAs to esters produces water. He says that removal of this water might be necessary for the reaction to proceed to completion and he says this can be accomplished by absorption with sodium sulfate or by heating. He also observed that transesterification reactions with higher alcohols, beyond methanol, are especially sensitive to water.

Arrowsmith makes a number of additional observations about the alkyl ester production process. He notes that it is desirable to minimize the amount of alkali catalyst used because the amount of soap formed increased with increasing catalyst. He also observed that increasing the amount of excess alcohol could decrease the amount of catalyst required. However, excess alcohol can cause problems with separation of the esters and glycerol. This problem is particularly severe for ethanol and higher alcohols. The separation process can be enhanced by adding water, which will pull the glycerol out of the ester phase. However, now the lower phase is a mixture of glycerol, alcohol, and water. Considerable extra energy is required to separate and purify these three compounds, especially if they involve azeotropes as is the case with ethanol and water. Arrowsmith proposes to solve this problem with a water-free process. 
Aug. 28, 1945.

H. D. ALLEN ET AL

$2,383,579$

PROCESS FOR TREATING FATS AND FATTY OILS

Filed March 30, 1943

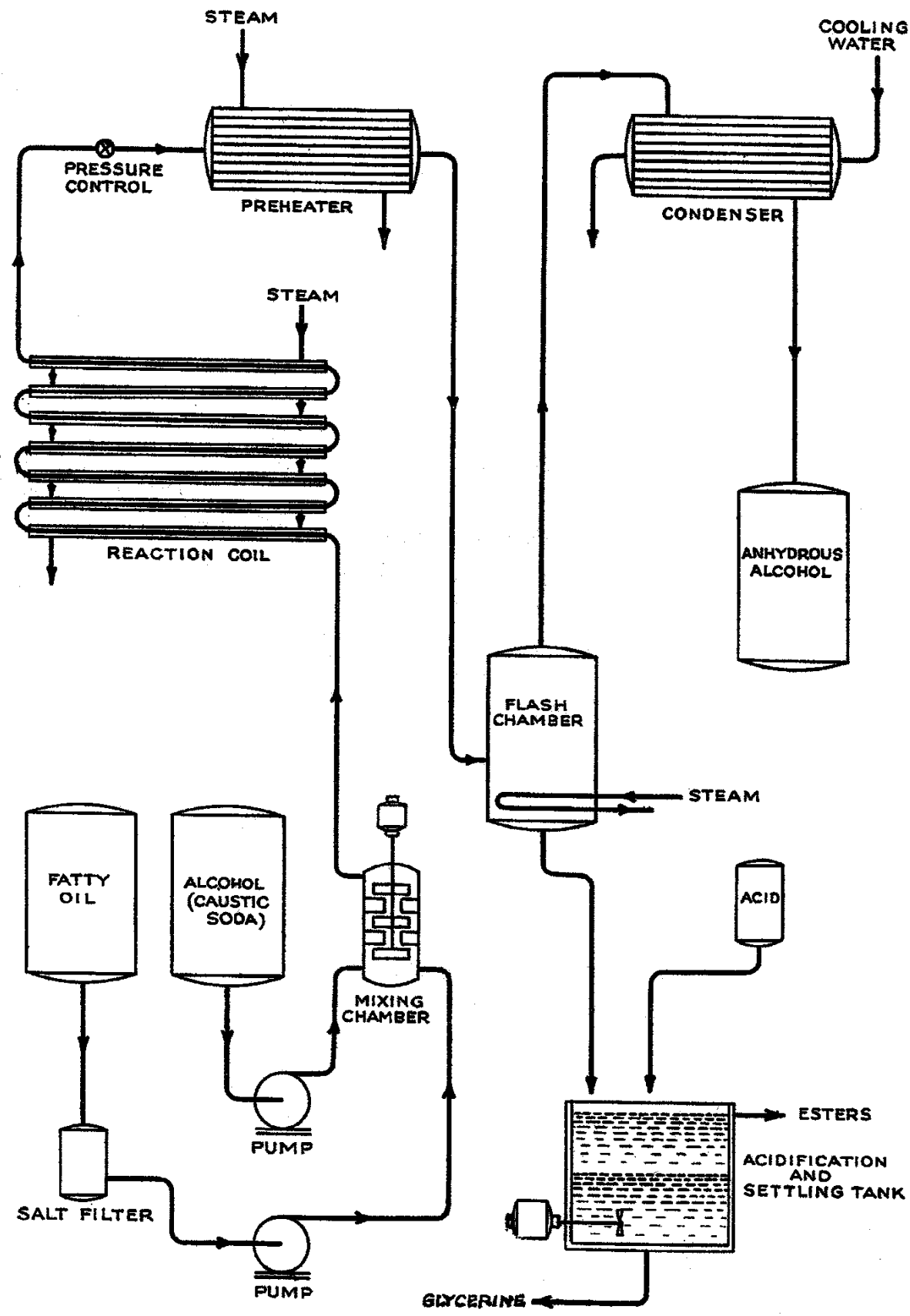

Figure 22. Process Flow Diagram by H.D. Allen, Patent No. 2,383,579. 
Instead of using a washing process to purify the esters, he recommends a vacuum distillation. According to Arrowsmith, the esters and glycerol form an azeotrope with a boiling temperature that is lower than the boiling temperature of either pure component. Distillation is performed at pressures of 1 to $15 \mathrm{~mm} \mathrm{Hg}$. Arrowsmith states that the transesterification reaction can be conducted at temperatures as high as $250 \mathrm{C}$, but that at temperatures above about $140-160 \mathrm{C}$, the soap can catalyze the reverse reaction and break up the esters to free fatty acids and alcohol. By staying at $130 \mathrm{C}$, it was possible to attain 30 second reaction times and achieve $97 \%$ complete reaction.

Trent also noted the tendency of excess alcohol to serve as a cosolvent and inhibit the separation of the ester and glycerol. Trent developed an apparatus for removing the alcohol and separating the esters and glycerol in a single device. Two variations of the device are shown in Figure 23. The first device (left side) shows a system where the oil and alcohol/catalyst mixture are mixed and passed through a coil of sufficient length to allow the reaction to reach completion before entering a chamber that is heated by a steam coil. The entire process is at or near atmospheric pressure. The temperature of the chamber is high enough that the alcohol boils off leaving the mixture of esters and glycerol. Acid is added to neutralize the mixture and split the soap into free fatty acids and salts. Then, the ester/glycerol mixture passes through a tube into the lower half of the chamber where it separates. Esters are removed from the top of the lower chamber and the glycerol is removed from the bottom.

The second variation (right side) is similar except that the reaction is conducted at higher temperature and pressure. Instead of a steam coil to boil off the alcohol, the pressurized mixture is sprayed into the upper half of the container, which acts as a flash chamber. Vaporized alcohol is removed and the ester/glycerol enters the bottom half of the container where it separates as with the other device.

Trent mentions other novel aspects of the alcoholysis process: Trent says that esters produced with higher alcohols (ethanol and above) have a greater tendency to gel from soap formation than with methanol. He claims that better yields are found if reactants are mixed cold and then heated, instead of preheated and then mixed. He says that early increases in temperature increase saponification and recommends using a step-wise increase in temperature from $40-50^{\circ} \mathrm{C}$ then up to 110 to $123^{\circ} \mathrm{C}$. He also mentions water washing using either batch, concurrent, or countercurrent flow systems. In addition to water washing, Trent claims that a liquid-liquid extraction process using a selective solvent that is at least partially immiscible with the esters can be used. He specifically suggests polar solvents such as furfural, sulfur dioxide, nitromethane, methanol, ethanol, ethylene glycol, allyl alcohol, ethyl sulfate, acetaldehyde, acetamide, dichlorodiethyl ether, and methyl carbitol. Trent notes that alcohol can be used as a washing agent for the oil to remove free fatty acids and water.

Percy points out that since alcohol is immiscible with the triglyceride feedstock and the final glycerol product is immiscible with the ester layer, this requires a high level of agitation to prevent saponification (soap formation) and separation of the glycerol layer and consequent removal of the alcoholysis catalyst. However, when mono- and diglycerides are present, the reaction mixture will be homogeneous. Percy advocates a process where only enough alcohol or 
Aug. 28, 1945.

W. R. TRENT

$2,383,632$

PROCESS OF TREATING FATMY GLYCERIDES

Filed Oct. 17, 1942

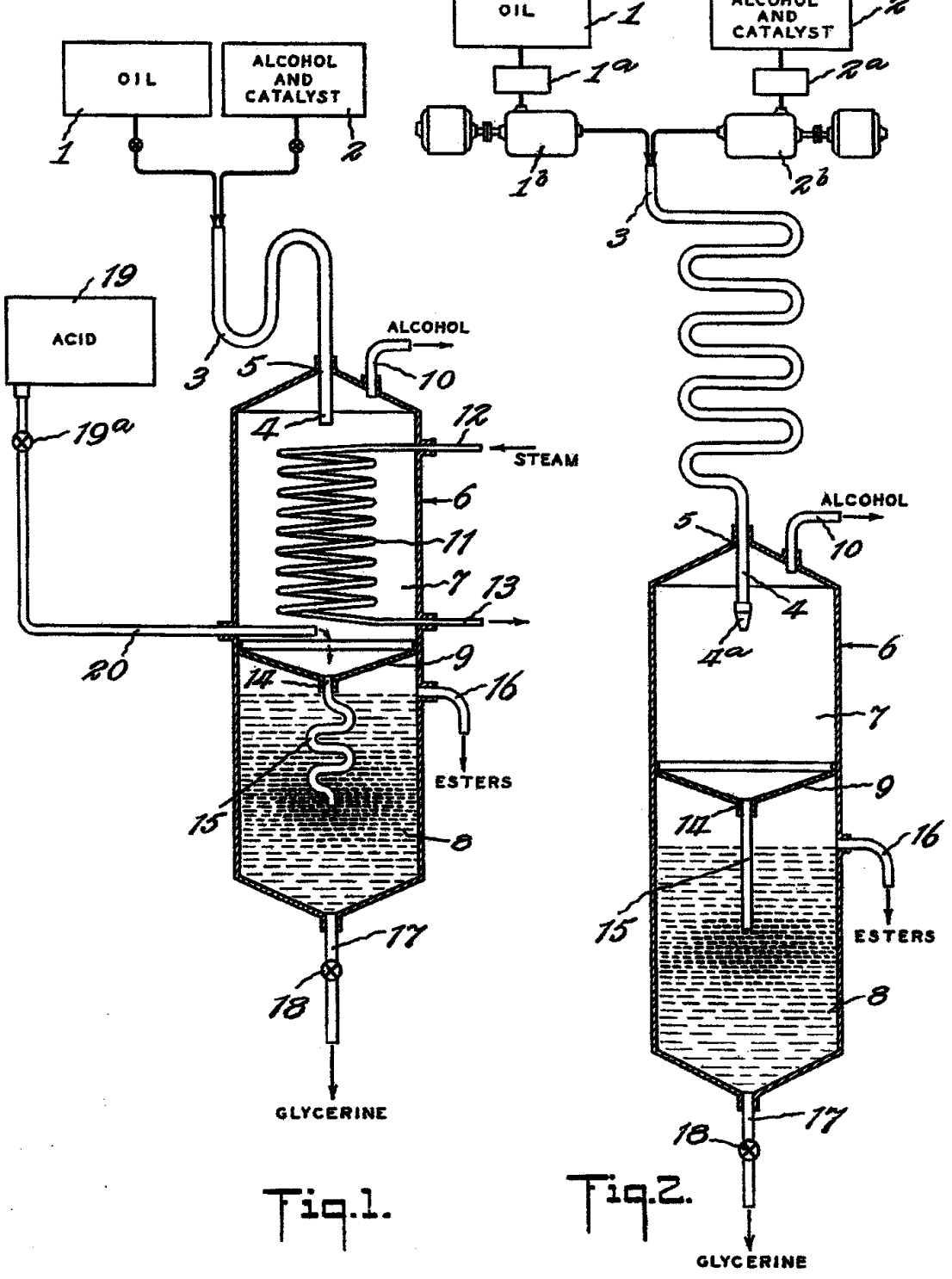

Figure 23. Trent's Combined Reactor and Glycerol Separator 
catalyst is added to form mono- and diglycerides. This reaction mixture will be homogeneous. This partially reacted mixture can be held and a stream bled off to which more alcohol and catalyst are added to complete the reaction. Percy claims that if the reaction is conducted at 10 atm and $175^{\circ} \mathrm{C}$, the reaction will proceed to completion without catalyst. When using higher alcohols, if ester/glycerol separation is a problem, adding methanol can help encourage the separation.

Dreger observes that ethanol and higher alcohols allow the use of higher reaction temperatures but he says that this increases the tendency toward soap formation. He advocates lower reaction temperatures of around $40^{\circ} \mathrm{C}$. In addition, he recommends the use of small amounts of methanol to ensure complete reaction and to promote separation of glycerol when using ethanol and higher alcohols. Adding 10-30\% methanol to whatever alcohol is being used will encourage complete reaction and ester/glycerol separation.

\section{Patents Referenced:}

Bradshaw (1942, 1944): E.I. duPont de Nemours \& Company, 2,271,619 and 2,360,844 Allen (1945): Colgate-Palmolive-Peet, 2,383,579

Arrowsmith (1945): Colgate-Palmolive-Peet, 2,383,580 and 2,383,581

Percy (1945): Colgate-Palmolive-Peet, 2,383,614

Keim (1945): Colgate-Palmolive-Peet, 2,383,601 and 2,383,602

Trent (1945): Colgate-Palmolive-Peet, 2,383,632 and 2,383,633

Dreger (1945): Colgate-Palmolive-Peet, 2,383,596 


\section{Patent List for Biodiesel}

\section{The following patents relate to the production, processing, and utilization of biodiesel.}

Arrowsmith, C.J. and J. Ross, "Treating Fatty Materials," US Patent 2,383,580. 1945.

Assmann, G., G. Blasey, B. Gutsche, L. Jeromin, J. Rigal, R. Armengaud and B. Cormary, "Continuous process for the production of lower alkyl esters", U. S. Patent \# 5,514,820, May 7, 1996.

Bam, N., Drown, D.C., Korus, R., Hoffman, D.S., Johnson, T.G., and Washam, J.M., "Method for Purifying Alcohol Esters," US Patent 5424467, June 13, 1995.

Barnhorst, J. A., M D. Staley and D. A. Oester, “Transesterification process", U.S. Patent 6,489,496, December 3, 2002.

Basu, H.N. and M.E. Norris, "Process for Production of Esters for Use as a Diesel Fuel Substitute Using a Non-Alkaline Catalyst," US Patent 5525126, June 11, 1996.

Bayense, C. R., H. Hinnekens and J. Martens, "Esterification process", U. S. Patent 5,508,457, April 16, 1996.

Billenstein, S., B. Kukla and H. Stuhler, "Process for the preparation of fatty acid esters of shortchain alcohols", U. S. Patent 4,668,439, May 26, 1987.

Bollman, H. 1921. Process for the Removal of Fatty Acids, Resins, Bitter and Mucilaginous Substances from Fats and Oils. U.S. Patent 1,371,342.

Bradin, D.S., "Biodiesel Fuel," US Patent 5578090, November 26, 1996.

Bradshaw, G.B., “New Soap Process,” Soap, V. 18, May 1942, pp. 23-24, 69-70.

Bradshaw, G.B. and W.C. Meuly. 1942. Process of Making Pure Soaps. U.S. Patent 2,271,619.

Bradshaw, G.B. and W.C. Meuly, "Preparation of Detergents," U.S. Patent No. 2,360,844 Awarded Oct. 24, 1944.

Buxton, L.O., "Process for Treating Fatty Oils," US Patent 2,345,097. 1944.

Colbeth, I. M., "Process of reacting glyceride oils”, U. S. Patent 2,469,371, May 10, 1949.

Connemann, J., A. Krallmann, and E. Fischer, "Process for the continuous production of lower alkyl esters of higher fatty acids", U.S. Patent 5,354,878, October 11, 1994.

Demmering, G., C. Pelzer and L. Friesenhagen, "Process for the production of fatty acid lower alkyl esters", U. S. Patent 5,455,370, October 3, 1995. 
Feldman, J. and J.M. Hoyt, "Extractive Distillation of Alcohol-ester mixtures," US Patent 4,473,444. 1984.

Foglia, T.A., L.A. Nelson, W.N. Marmer, "Production of biodiesel, lubricants and fuel and lubricant additives," US Patent 5,713,965. 1998.

Glossop, G. A., “Treating Fatty Glycerides”, U.S. Patent 2,383,599, August 28, 1945.

Granberg, E. P., R. G. Schafermeyer and J. A. Letton, "Method for purifying an inert gas while preparing lower alkyl esters", U. S. Patent 5,844,111, December 1, 1998.

Haas, M. J., S. Bloomer and K. Scott, "Process for the production of fatty acid alkyl esters", U.S. Patent 6,399,800, June 4, 2002.

Hay, R. G., J. G. McNulty, W. L. Walsh, "Process for preparing esters", U.S. Patent 3,692,822, September 19, 1972.

Hunt, T. K., L. Jeromin, W. Johannisbauer, B. Gutsche, V. Jordon and H. Wogatzki, "Recovery of tocopherols", U. S. Patent 5,646,311, July 8, 1997.

Jeromin, L., E. Peukert and G. Wellmann, "Process for the pre-esterification of free fatty acids in fats and oils", U. S. Patent 4,698,186, October 6, 1987.

Jeromin, L., E. Peukert, B. Gutsche, G. Wollman, and B. Schleper, "Process for the continuous transesterification of fatty acid lower alkyl esters" U. S. Patent 4,976,892, December 11, 1990.

Johnson, L.A. and E.G. Hammond, "Soybean Oil Ester Fuel Blends," U.S. Patent 5,520,708, May 28, 1996.

Kawahara, Y. and T. Ono, "Process for Producing Lower Alcohol Esters of Fatty Acids," U.S. Patent 4,164,506. Awarded Aug. 14, 1979.

Keim, G.I., "Treating Fats and Fatty Oils,” U.S. Patent 2,383,601, Awarded Aug. 28, 1945.

Kesling Jr., H. S., L. J. Karas, and F. J. Liotta Jr., “Diesel fuel”, U. S. Patent 5,308,365, May 3, 1994.

Klok, R and H. H. Verveer, "Process for producing fatty-acid lower-alkyl mono-esters", U. S. Patent 5,116,546, May 26, 1992.

Kuceski, V. P., "Purification of alpha, omega alkanedioic acids by partial esterification", U. S. Patent 2,824,122, February 18, 1958. 
Lepper, H. and L. Friesenhagen, "Process for the production of fatty acid esters of short-chain aliphatic alcohols from fats and/or oils containing free fatty acids", U. S. Patent 4,608,202, August 26, 1986.

Lepper, H. and L. Friesenhagen, "Process for the production of fatty acid alkyl esters", U. S. Patent 4,652,406, March 24, 1987.

Matsukura, T. and Y. Nakagawa, "Method for Manufacturing High Quality Fatty Acid Esters," US Patent 4,371,470 February 1, 1983.

Matsumoto, W., E. Nakai, T. Nezu, and K. Suzuki, "Process for the Transesterification of fat and oil," US Patent 5,089,404. 1992.

Mittelbach, M., "Method for the Preparation of Fatty Acid Alkyl Esters," US Patent 5849939, Dec. 15, 1998.

Nourreddini, H., "Process for Producing Biodiesel Fuel with Reduced Viscosity and a Cloud Point Below 32 deg. Fahrenheit," U.S. patent 6,015,440, January 18, 2000.

Noureddini, H., "System and process for producing biodiesel fuel with reduced viscosity and a cloud point below thirty-two (32) degrees fahrenheit”, U. S. Patent 6,174,501, January 16, 2001.

Peter, S., R. Ganswindt and E. Weidner, "Method for producing fatty acid esters", U. S. Patent 6,211,390, April 3, 2001.

Reierson, R.L., "Process for Removing Glycerin," US Patent 4,360,407. 1982.

Schmerling, L., "Transesterification of Carboxylic Acids," US Patent 4,112,235. 1978.

(tin halide catalyst)

Sprules, F. J. and D. Price, "Production of fatty esters", U.S. Patent 2,494,366, January 10, 1950.

Stage, H., "Process for Deodorizing and/or Physical Refining of high-Boiling Organic Edible Oils, Fats, and Esters," U.S. Patent 4,599,143, July 8, 1986.

Stage, H., "Process for Degassing, Dehydrating, and Precut Separation in Straight-run Distillation of Crude Fatty Acids," U.S. Patent No. 4,680,092, July 14, 1987.

Stern, R., G. Hillion, P. Gateau, and J.-C. Guibet, "Process for Manufacturing a Composition of Fatty Acid Esters Useful as Gas Oil Substitute Motor fuel with Hydrated Ethyl Alcohol and the Resultant Esters Composition," U.S. Patent 4,695,411. Awarded Sept. 22, 1987.

Stern, R., G. Hillion, M.N. Eisa, "Process for the Production of Ethyl Esters," U.S. Patent 6,013,817, Awarded Jan. 11, 2000. 
Stern, R., G. Hillion, J. J. Rouxel, and S. Leporq, "Process for the production of esters from vegetable oils or animal oils alcohols", U. S. Patent 5,908,946, June 1, 1999.

Stern, R., G. Hillion and J. J. Rouxel, "Improved process for the production of esters from fatty substances having a natural origin”, U. S. Patent 5,424,466, June 13, 1995.

Stidham, W. D., D. W. Seaman, M. F. Danzer, "Method for preparing a lower alkyl ester product from vegetable oil”, U. S. Patent 6,127,560, October 3, 2000.

Tanaka, Y., A. Okabe and S. Ando, "Method for the preparation of a lower alkyl ester of fatty acids", U. S. Patent 4,303,590, December 1, 1981.

Trent, W.R., "Process of Treating Fatty Glycerides," US Patent 2,383,632. 1945.

Trent, W.R., "Process for Treating Fatty Glycerides," US Patent 2,383,633. 1945

Venter, J. J. and C. McDade, “Transesterification catalyst”, U. S. Patent 5,183,930, February 2, 1993.

Wimmer, T., "Process for the Production of Fatty Acid Esters of Lower Alcohols," US Patent 5399731, March 21, 1995.

Wimmer, T., "Process for Preparing Fatty Acid Esters of Short-Chain Monohydric Alcohols," US Patent 5,434,279. 1995.

$\mathrm{Wu}, \mathrm{W}$. T. and J. W. Chen "Method of preparing lower alkyl fatty acids esters and in particular biodiesel”, U. S. Patent 6,398,707, June 4, 2002.

Yeh, L. I., R. H. Schlosberg, R. C. Miller, J. R. Bateman, R. F. Caers, “Diesel fuel composition”, U.S. Patent 6,447,557, September 20, 2002.

Yeh, L. I., R. H. Schlosberg, R. C. Miller, J. R. Bateman, R. F. Caers, "Diesel fuel composition”, U.S. Patent 6,458,176, October 1, 2002.

Yeh, L. I., R. H. Schlosberg, R. C. Miller, J. R. Bateman, R. F. Caers, “Diesel fuel composition”, U.S. Patent 6,447,558, September 10, 2002. 


\section{Post Reaction Processing}

\section{Introduction}

The objective of this section is to describe in more detail the steps in processing the ester phase resulting from the transesterification. This section will discuss the recovery of the esters from the reaction mixture, and, then, the refining needed to meet the requirements of ASTM D 6751-2. The topics include: ester/glycerol separation, ester washing, ester drying, other ester treatments, and additization.

\section{Ester/Glycerol Separation}

The ester/glycerol separation is typically the first step of product recovery in most biodiesel processes. The separation process is based on the facts that fatty acid alcohol esters and glycerol are sparingly mutually soluble, and that there is a significant difference in density between the ester and glycerol phases. The presence of methanol in one or both phases affects the solubility of ester in glycerol and glycerol in ester.

The ester washing step is used to neutralize any residual catalyst, to remove any soaps formed during the esterification reaction, and to remove residual free glycerol and methanol.

Ester drying is required to meet the stringent limits on the amount of water present in the final biodiesel product. In addition, there may be other treatments used to reduce color bodies in the fuel, remove sulfur and/or phosphorus from the fuel, or to remove glycerides.

Additization is the addition of materials the have a specific functionality that modifies one or more fuel properties. Examples include cloud point/pour point additives, antioxidants, or other stability enhancing agents.

Fatty acid alcohol esters have a density of about $0.88 \mathrm{gm} / \mathrm{cc}$, while the glycerol phase has a density on the order of $1.05 \mathrm{gm} / \mathrm{cc}$, or more. The glycerol density depends on the amount of methanol, water, and catalyst in the glycerol. This density difference is sufficient for the use of simple gravity separation techniques for the two phases.

However, the rate of separation is affected by several factors. Most biodiesel processes use relatively intense mixing, at least at the beginning of the reaction, to incorporate the sparingly soluble alcohol into the oil phase. If this mixing continues for the entire reaction, the glycerol can be dispersed in very fine droplets throughout the mixture. This dispersion requires from one hour to several hours to allow the droplets to coalesce into a distinct glycerol phase. For this reason, mixing is generally slowed as the reaction begins to progress, to reduce the time required for phase separation.

The more nearly neutral the $\mathrm{pH}$, the quicker the glycerol phase will coalesce. This is one reason to minimize the total catalyst use. In some batch systems the reaction mixture is neutralized at the beginning of the glycerol/ester phase separation step. 
The presence of significant quantities of mono-, di-, and triglycerides in the final mixture can lead to the formation of an emulsion layer at the ester - glycerol interface. At best, this layer represents a net loss of product, unless it is recovered and separated. At worst, the ester phase will not meet the biodiesel specification and will have to be re-run. If problems with mono, di, and triglycerides occur, you should reevaluate the entire reaction to see where improvements can be made to improve process yields in the preceding steps.

The esterification process is run with an excess of alcohol to ensure complete reaction and to attain higher reaction rates. The residual alcohol is distributed between the ester and glycerol phases. The alcohol can act as a dispersant for the ester into the glycerol phase and for the glycerol into the ester phase. The result can be a need for additional processing of the products to meet specifications. Other people claim that methanol aids in phase separation, which is one reason that product is generally phase separated before methanol recovery.

\section{Process Equipment for the Ester/Glycerol Separation}

Decanter Systems: There are three categories of equipment used to separate the ester and glycerol phases. Decanter systems rely solely on the density difference and residence time to achieve the separation. For relatively small throughput, or batch processes, the 1 to 8 hours required for complete separation of the phases may be acceptable. However, a separation that requires a residence time of 1 hour requires a decanter with a volume of at least 700 gallons to affect separation for a 5,000,000 gallon per year, continuous plant. For lower extent of reaction, the separation is slower, and the decanter will have to be much larger.

The primary determinant for designing a decanter for biodiesel production is the desired residence time. This, plus the product mixture flow rate determines the size of the unit. Decanter units should be rather tall and narrow to allow physical separation between the ester and the glycerol withdrawal points. L/D ratios of 5 to 10 can work well.

The temperature in the decanter affects the solubility of the alcohol in both phases, and the viscosity of the two liquids. Too high a temperature in the decanter can cause residual alcohol to flash, potentially restricting the flow of the ester phase out of the tank. On the other hand, too low a temperature increases the viscosity in both phases. The increased viscosity will slow the coalescence rate in the system.

The presence of an emulsion layer is indicative of mono- and di-glycerides. The emulsion layer will form between the phases. In continuous operation, there must be a provision for removing the emulsion, so it does not fill the decanter.

Centrifuge System: Many of the continuous plants use a centrifuge for the phase separation. The centrifuge creates an artificial, high gravity field by spinning at very high speeds. The separation can be completed rapidly and effectively. The disadvantage of the centrifuge is its initial cost, and the need for considerable and careful maintenance. However, centrifuges are used extensively in the food processing, rendering, and biodiesel industries. 
Centrifuges are high-speed, rotating devices. The artificial gravity of the centrifuge results from the rotation. Speeds of 2000 to $5000 \mathrm{rpm}$ are not uncommon. The same high- speed rotation that creates the centrifugal effect also creates a device that requires careful and rather frequent maintenance. Although centrifuges are relatively expensive, multiple units to ensure on-line availability are advisable.

At smaller capacities, either a batch or a continuous centrifuge can be used. The use of a batch centrifuge in a continuous process requires a surge tank to match the batch cycle time with the continuous processing rate.

Hydrocyclone: An intriguing, relatively new device that has been considered for use in biodiesel plants is the hydrocyclone. A liquid - liquid hydrocyclone uses an inverted conical shape and the incompressibility of the liquids to accelerate the liquid entering the cyclone. The effect is similar to a centrifuge, with the heavier material being forced towards the wall and downward, and the lighter material forced to the center and upward. The result is a density-based separation. Although they are now used for oil - water separations, hydrocyclones are at the experimental stage for application in biodiesel production.

A hydrocyclone operates on the Bernoulli principle of trading pressure for speed in an incompressible flowing system. The relative density of the fluids determines the separating force applied, while the relative viscosity determines the resistance to separation. The liquid mixture enters the hydrocyclone at a moderately high pressure (about $125 \mathrm{psig}$ ). The pressure decreases and the velocity increases as the liquid passes for the wider to the narrower part of the inverted cone. The more dense liquid is accelerated towards the outside wall, while the less dense liquid concentrates in the center. The result is a separation based upon induced g-forces.

It would appear that the presence of volatiles creates a problem in a hydrocyclone. The rapid reduction of pressure in the device will induce flashing of the volatile liquid (alcohol), disrupting or stopping the separation process. Excess methanol should be removed from the system before introducing the reaction mixture to a hydrocyclone.

\section{Ester Washing}

The primary purpose of the ester washing step is the removal of any soaps formed during the transesterification reaction. In addition, the water provides a medium for addition of acid to neutralize the remaining catalyst and a means to remove the product salts. The residual methanol should be removed before the wash step. This prevents the addition of methanol to the wastewater effluent. However, some processes remove the methanol with the wash water and remove it from the wash water.

The use of warm water $\left(120\right.$ to $\left.140{ }^{\circ} \mathrm{F}\right)$ prevents precipitation of saturated fatty acid esters and retards the formation of emulsions with the use of a gentle washing action.

Softened water (slightly acidic) eliminates calcium and magnesium contamination and neutralizes remaining base catalysts. Similarly, removal of iron and copper ions eliminates a 
source of catalysts that decrease fuel stability. Gentle washing prevents the formation of emulsions and results a rapid and complete phase separation.

The phase separation between esters and water is typically very clean and complete. However, the equilibrium solubility of water in esters is higher than the specified water content for B100. Therefore, after the washing step there will be more than the equilibrium amount of water present.

Vacuum driers can either be batch or continuous devices for removing water. The system is operated at a highly reduced pressure, which allows the water to evaporate at much lower temperature. A variation that also allows for rather high heating and evaporation rates is the falling film evaporator. This device operates at reduced pressure. As the esters pour down the inside wall of the evaporator the direct contact with the heated wall evaporates the water rapidly. Care should be taken with high temperature evaporators to avoid darkening the fuel which is a sign that the polyunsaturated methyl esters are polymerizing.

Because the total water burden in the esters is low, molecular sieves, silica gels, etc. can also be used to remove the water. An advantage of these systems is that they are passive. However, a disadvantage is that these units must be periodically regenerated.

\section{Other Ester Treatments}

There are absorbents on the market that selectively absorb hydrophyllic materials such as glycerol and mono- and di-glycerides (i.e Magnesol from the Dallas Group). This treatment, followed by an appropriate filter, has been shown to be effective in lowering glycerides and total glycerol levels.

Some vegetable oils and many yellow greases and brown greases leave an objectionable color in the biodiesel. There is no color specification in ASTM D 6751, but an activated carbon bed is an effective way to remove excessive color. The fats and oil industry literature has other bleaching technologies that may also be explored for biodiesel producers.

The European specification for sulfur content is much tighter than the U.S. requirement. As a result, a number of producers in Europe are resorting to the use of vacuum distillation for the removal of sulfur compounds from the biodiesel product. By 2006, all U.S. biodiesel must meet new sulfur standards of $15 \mathrm{ppm}$ or less. Therefore, biodiesel producers needs to be aware of the sulfur content of their fuel, and must incorporate sulfur reduction technology if needed before that date. Vacuum distillation has the added benefit of deodorization and the removal of other minor contaminants, which may provide a benefit to those firms that use highly degraded feedstocks such as trap grease.

Filtering is an essential part of all biodiesel production. While feedstocks entering the plant should be filtered to at least $100 \mu \mathrm{m}$, biodiesel leaving the plant should be filtered to at least 5 $\mu \mathrm{m}$ to ensure no contaminants are carried with the fuel that could damage the engine. At has been suggested that the fuel could be cooled before filtering to capture some of the saturated 
esters as they crystallize and thereby lower the cloud point of the fuel. The crystallized esters could be melted by heating and used within the plant as boiler fuel.

\section{Additization of Esters}

Petroleum-based diesel fuels are treated with a wide range of additives to improve lubricity, detergency, oxidative stability, corrosion resistance, conductivity, and many other properties. Additive technology for biodiesel is less advanced, so fewer additives are available to enhance performance.

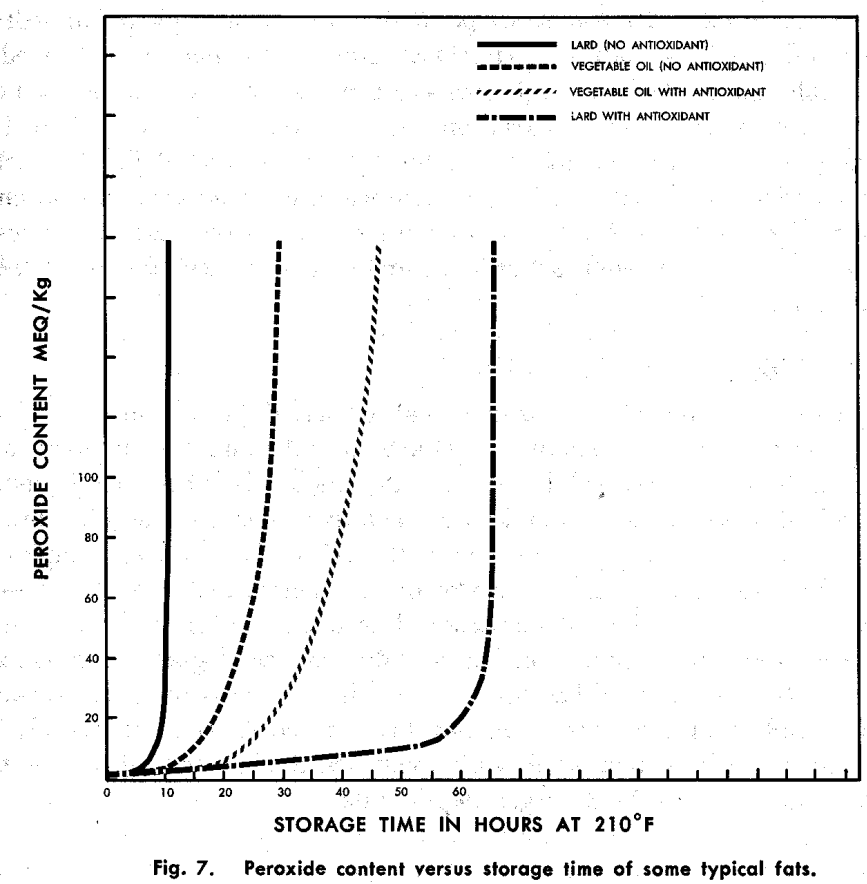

Figure 24. Oxidation behavior of vegetable oils and animal fats [1].

One area where the biodiesel producer needs to give serious consideration is oxidative stability. Biodiesel, because it contains large numbers of molecules with double bonds, is much less oxidatively stable than petroleum-based diesel fuel. Fortunately, stability-enhancing additive technology is well-developed in the food industry and many of these additives can be carried over to stabilizing biodiesel.

Figure 24 shows typical oxidation behavior for vegetable oils and animal fats. In this test, the oil and fat were exposed to heat and aeration to accelerate the oxidation process. The progress of the oxidation is monitored by measuring the peroxide value, or the fraction of the oil that has been converted to a peroxide molecule, the first step in the oxidation process. Both the vegetable oil and animal fat have no conversion for a period of time (called the induction period), but then oxidize quickly. In this case, the lard actually oxidizes earlier than the vegetable oil, probably due to the presence of natural antioxidants in the vegetable oil. Lipid oxidation is an autocatalytic process so that when it starts, it progresses at ever-increasing rate. 


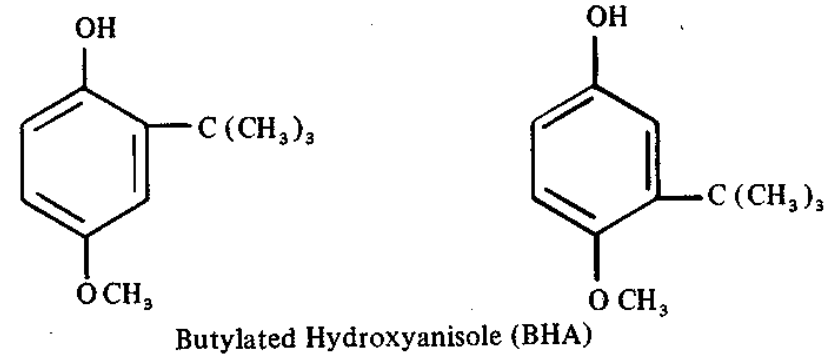

3-BHA (3-tert-butyl-4-hydroxyanisole;

2-tert-butyl-4-methoxyphenol)

2-BHA (2-tert-butyl-4-hydroxyanisole 3-tert-butyl-4-methoxyphenol)<smiles>Cc1cc(CC(C)(C)C)c(O)c(CC(C)(C)C)c1</smiles>

Butylated Hydroxy toluene (BHT)<smiles>CC(C)(C)c1cc(O)ccc1O</smiles>

Mono-tert-butylhydroquinone (TBHQ)

Figure 25. Antioxidants for oils and fats [2]. 


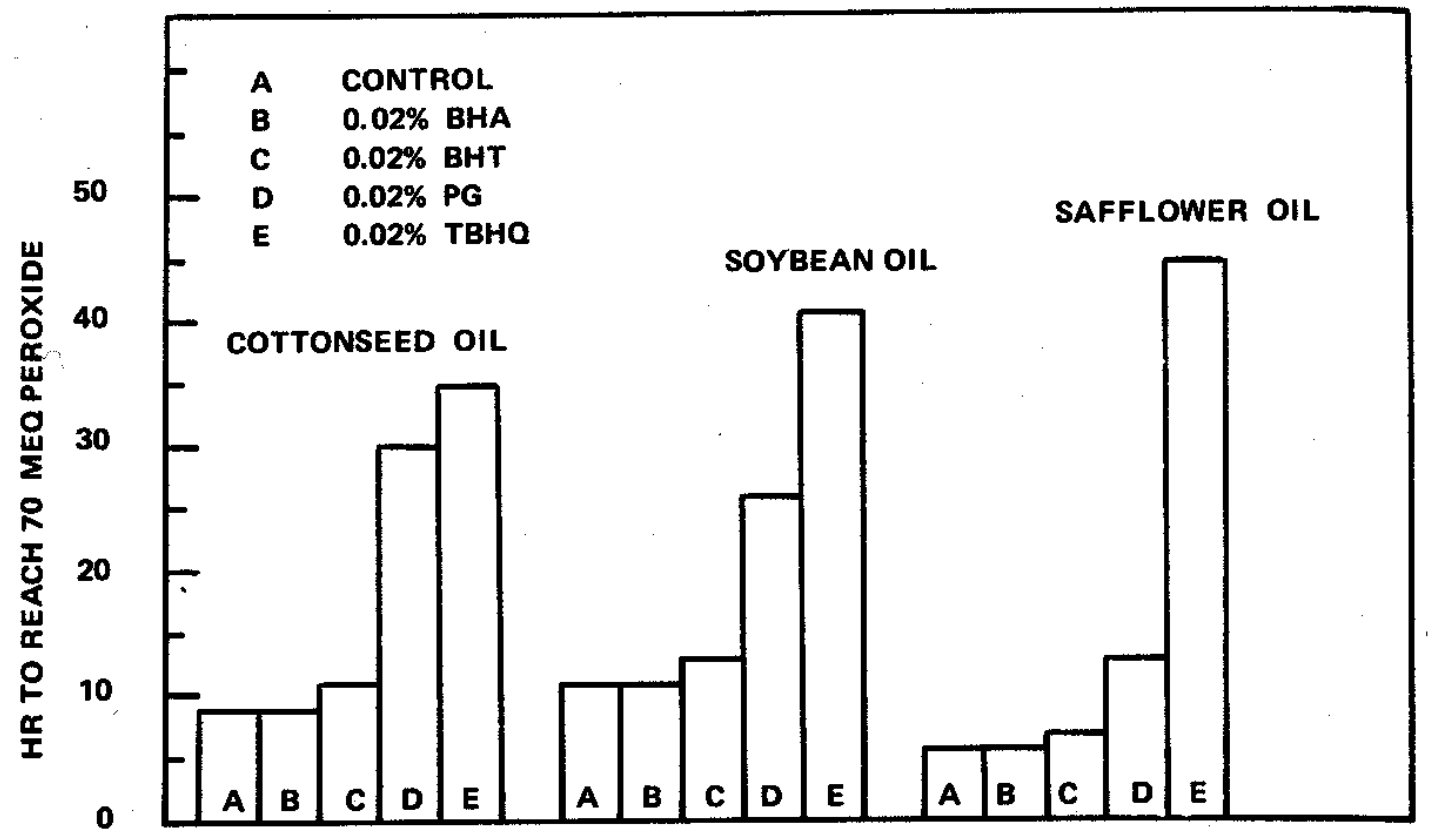

Figure 26. Comparison of tert-butylhydroquinone (TBHQ) with other anioxidants by the active oxygen method (AOM). (From Sherwin, E. R. and Thompson, J. W., Food Technol. (Chicago), 21(6), 106, 1967.

Figure 25 shows some of the most common antioxidant additives used in food products: BHA, BHT, PG, and TBHQ. The properties of these additives are well known and they are considered safe.

Figure 26 shows a comparison of the effectiveness of the different antioxidants in three different oils. This data was obtained from oils and not biodiesel, however, since the oxidation processes are similar for the two classes of compounds, the relative effectiveness of the additives should be similar. Figure 26 clearly shows that TBHQ is the most effective additive for equivalent treat rates.

Table 8 shows additional comparisons between the additives for variable treat rates. Again, TBHQ is almost twice as effective as the nearest other additive.

Since metals, especially copper, can act as catalysts for oxidation, compounds called chelators are frequently added to the oils and fats along with the antioxidants. Chelators bind up metals into stable molecules that do not participate in promoting oxidation. Citric acid is a common chelator used in the food industry [1]. Figure 4 shows the effect of adding citric acid to antioxidants. It extends the oxidative induction period and improves the stability of the oil. 
Table 8. Comparison of TBHQ with BHA, BHT, and PG in Vegetable Oils by AOM and Oven Storage Tests.

\begin{tabular}{|c|c|c|c|c|c|c|}
\hline \multirow{2}{*}{$\begin{array}{l}\text { Oil treatment (wt \% } \\
\text { cone) }\end{array}$} & \multicolumn{3}{|c|}{$\begin{array}{l}\text { AOM stability (hr to reach } 70 \mathrm{meq} \\
\text { peroxide content) }\end{array}$} & \multicolumn{3}{|c|}{$\begin{array}{l}\text { Storage life at } 115^{\circ} \mathrm{F} \text { of potato chips } \\
\text { prepared with oils (days) }\end{array}$} \\
\hline & Cottonseed & Soybean & Safflower & Cottonseed & Soybean & Safflower \\
\hline Control (no antioxidant) & 9 & 11 & 6 & 11 & 8 & 4 \\
\hline $0.010 \mathrm{TBHQ}$ & 24 & 29 & 29 & 17 & $-{ }^{B}$ & $-^{b}$ \\
\hline $0.020 \mathrm{TBHQ}$ & 34 & 41 & 40 & 23 & 25 & $23+$ \\
\hline 0.030 TBHQ & 42 & 53 & 77 & 33 & $-\infty$ & $-^{a}$ \\
\hline $0.010 \mathrm{BHA}$ & 9 & 12 & 8 & 9 & $-{ }^{b}$ & $-{ }^{b}$ \\
\hline 0.020 BHA & 9 & 10 & 6 & 10 & 9 & 7 \\
\hline $0.030 \mathrm{BHA}$ & 9 & 10 & 7 & 7 & $-^{*}$ & $-^{\circ}$ \\
\hline $0.010 \mathrm{BHT}$ & 10 & 12 & 10 & 12 & $-{ }^{*}$ & $-^{b}$ \\
\hline $0.020 \mathrm{BHT}$ & 11 & 13 & 7 & 7 & 10 & 9 \\
\hline $0.030 \mathrm{BHT}$ & 13 & 15 & 8 & 15 & $-{ }^{\bullet}$ & -+ \\
\hline $0.010 \mathrm{PG}$ & 19 & 21 & 13 & 12 & $-{ }^{\circ}$ & $-{ }^{b}$ \\
\hline $0.020 \mathrm{PG}$ & 30 & 26 & 10 & 14 & 20 & 7 \\
\hline $0.030 \mathrm{PG}$ & 37 & 31 & 12 & 17 & $-{ }^{\circ}$ & $-{ }^{\circ}$ \\
\hline
\end{tabular}




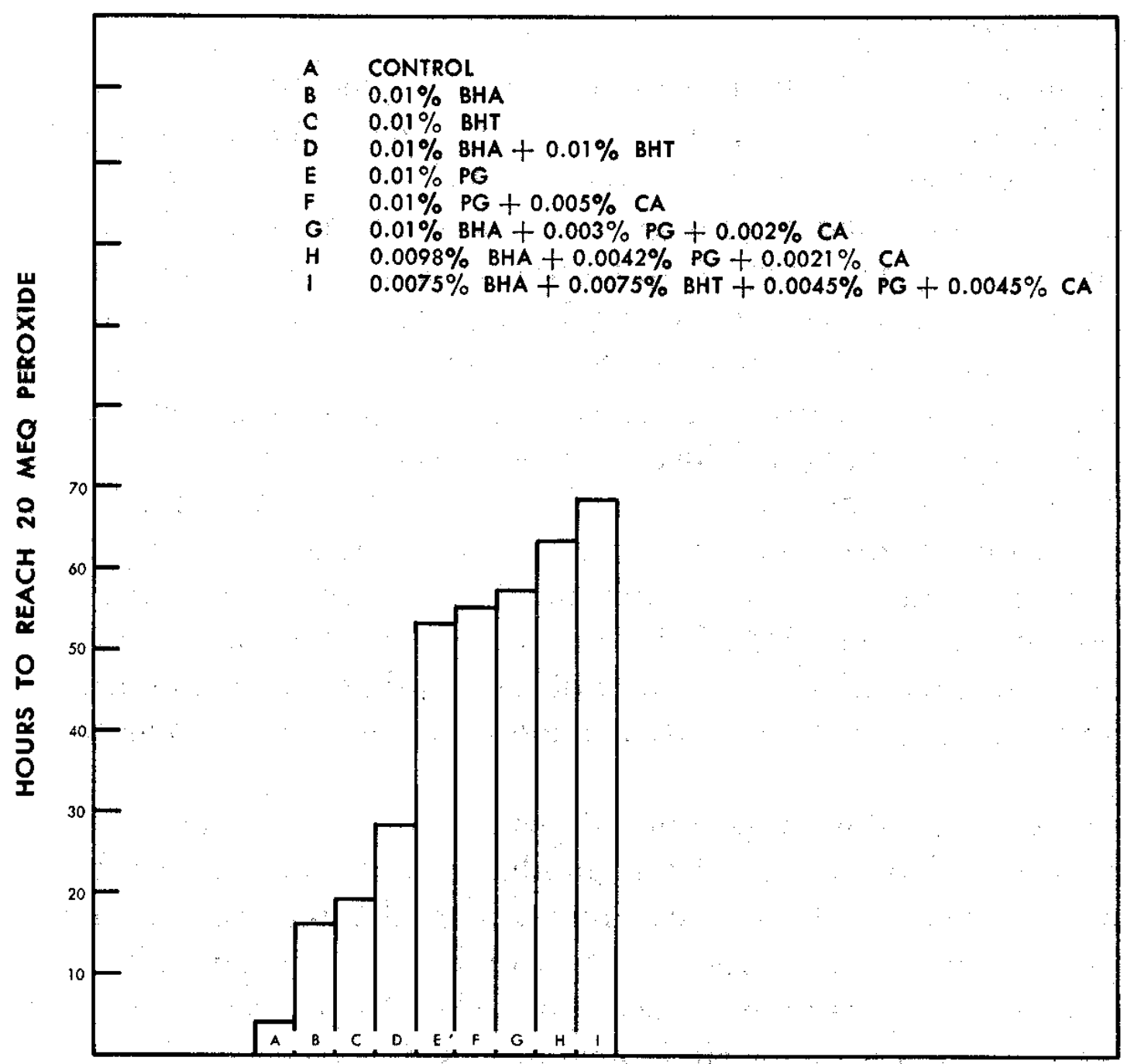

Figure 27. Effect of Citric Acid on Antioxidant Effectiveness

\section{References}

1. Furia, T.E., Editor, Handbook of Food Additives, CRC Press, Cleveland, OH, $2^{\text {nd }}$ Edition, 1972.

2. Furia, T.E., Editor, Handbook of Food Additives, Vol. 2, CRC Press, Cleveland, OH, $2^{\text {nd }}$ Edition, 1980. 


\section{Treatment and Recovery of Side Streams}

\section{Introduction}

There are three non-ester side streams that must be treated as a part of the overall biodiesel process. These streams are:

1) The excess alcohol that is recycled within the process;

2) The glycerol co-product, and;

3) The wastewater stream from the process.

In this module it is assumed that methanol is the alcohol used in the process. Similar comments would apply to other alcohols.

Methanol recycle is necessary because an excess of methanol is required for an effective transesterification reaction system. The recovery of the unused methanol saves input costs for the process and essentially eliminates the emissions of methanol to the surroundings. The emissions reduction is needed because methanol is highly flammable and is toxic.

Glycerol is recovered and partially refined as a co-product from biodiesel production. About 10 percent by weight of the input reactants are converted to glycerol in the transesterification reaction. On both a weight basis and a volume basis, partially refined glycerol is worth more than the biodiesel product.

Wastewater constitutes an operating cost for the plant, both because of the water consumption and because of the water treatment costs to the plant.

\section{Methanol Management}

There are several physical parameters that are important to the recovery and recycle of methanol. Methanol's relatively low boiling point, $64.7^{\circ} \mathrm{C}$, means that it is fairly volatile and can largely be removed from the oil, ester and aqueous streams by flash evaporation and re-condensation. The low boiling point, along with a low flash point, $8^{\circ} \mathrm{C}$, also mean the methanol is considered to be highly flammable.

Methanol is fully miscible with water and with glycerol. However, it has a low solubility in fats and oils (approx. $10 \% \mathrm{wt} / \mathrm{wt}$ at $65^{\circ} \mathrm{C}$ in tallow). Methanol is more soluble in esters, but it is not fully miscible. The solubility in glycerol and water means that methanol will prefer these phases when there is a two-phase system present. The low solubility in fats and oils is the reason for the solubility-limited phase of the overall transesterification reaction.

When the two phases present are esters and glycerol, the methanol will distribute between the phases. At 90:10\% wt/wt ester and glycerol, the methanol distributes approximately 60:40 wt \% between the phases. This fact is important, since the reaction is complete at 90:10 wt \%. 
If the methanol is allowed to remain in the system during phase separation, the methanol acts as a phase stabilizer, retarding the rate of gravity separation. It is advantageous to remove the methanol before phase separation.

Methanol can be recovered using distillation, either conventional or vacuum, or partially recovered in a single stage flash. An alternative to distillation is a falling-film evaporator. Residual methanol in the ester phase can be removed in the water wash step in ester postprocessing. Product esters are typically washed with warm $\left(140^{\circ} \mathrm{F}\right)$, softened water to remove soaps and residual methanol.

\section{Glycerol Refining}

The recovered glycerol from the transesterification reaction contains residual alcohol, catalyst residue, carry-over fat/oil and some esters. The glycerol from rendered feedstocks may also contain phosphatides, sulfur compounds, proteins, aldehydes and ketones, and insolubles (dirt, minerals, bone, or fibers)

Chemical Refining: There are several factors that are important in the chemical refining or glycerol. First, the catalyst tends to concentrate in the glycerol phase where it must be neutralized. The neutralization step leads to the precipitation of salts.

Also, the soaps produced in the esterification must be removed by coagulation and precipitation with aluminum sulfate or ferric chloride. The removal may be supplemented by centrifuge separation.

The control of the $\mathrm{pH}$ is very important because low $\mathrm{pH}$ leads to dehydration of the glycerol and high $\mathrm{pH}$ leads to polymerization of the glycerol. The glycerol may then be bleached using activated carbon or clay.

Physical Refining: The first step in physical refining is to remove fatty, insoluble or precipitated solids by filtration and/or centrifugation. This removal may require $\mathrm{pH}$ adjustment. Then the water is removed by evaporation. All physical processing is typically conducted at 150 $-200^{\circ} \mathrm{F}$, where glycerol is less viscous, but still stable.

Glycerol Purification: The final purification of glycerol is completed using vacuum distillation with steam injection, followed by activated carbon bleaching. The advantages of this approach are that this is a well-established technology. The primary disadvantage is that the process is capital and energy intensive. Vacuum distillation of glycerol is best suited to operations $>25$ tons per day.

Ion exchange purification of glycerol is an attractive alternative to vacuum distillation for smaller capacity plants. The ion exchange system uses cation, anion, and mixed bed exchangers to remove catalyst and other impurities. The glycerol is first diluted with soft water to a 15 to 35 percent glycerol-in-water solution. The ion exchange is followed by vacuum distillation or flash drying for water removal, often to an 85 percent partially refined glycerol. 
The advantage of this process is the fact that all purification takes place in the resin vessels so the system is suited to smaller capacity operations. The disadvantages are that the system is subject to fouling by fatty acids, oils and soaps. The system also requires regeneration of the beds producing large quantities of wastewater. Regeneration requires parallel systems to operate and regenerate simultaneously.

\section{Wastewater Considerations}

Ester washing produces about 1 gallon of water per gallon of ester per wash. All process water must be softened to eliminate calcium and magnesium salts and treated to remove iron and copper ions. The ester wash water will have a fairly high BOD from the residual fat/oil, ester, and glycerol.

The glycerol ion exchange systems can produce large quantities of low salt waters as a result of the regeneration process. In addition, water softening, ion exchange and cooling water blowdown will contribute a moderate dissolved salts burden.

The aggregate process waste waters should meet local municipal waste treatment plant disposal requirements, if methanol is fully recovered in the plant and not present in the wastewater. In many areas, internal treatment and recycle of the process water may lead to cost savings and easier permitting of the process facility.

\section{Summary}

Methanol affects all product recovery operations. The methanol must be fully recycled for best economy and for pollution prevention. Glycerol is an economically significant co-product that should be as fully refined as practicable. Properly managed, wastewaters can be treated in a municipal sewer system, but internal treatment and recycling should be considered. 


\section{Laboratory - Exercise 2}

\section{Pretreatment of High Free Fatty Acid Feedstocks}

Equipment needed:

Sulfuric acid

Methanol

Four $500 \mathrm{ml}$ Erlenmeyer flasks

One liter each of four feedstocks of varying FFA level

Four hot plate/stirrers

Scale (reads to $0.1 \mathrm{~g}$ )

Divide the class into four groups. Each group should have different feedstocks with varying free fatty acid levels.

Procedure:

- Measure the acid value of the feedstock. Remember that FFA\% is approximately equal to half the acid value.

- Put $200 \mathrm{~g}$ of the feedstock into a $500 \mathrm{ml}$ flask, heat to $60-65^{\circ} \mathrm{C}$.

- Measure out in a $125 \mathrm{ml}$ flask an amount of methanol equal to $2.25 \mathrm{~g}$ of methanol for every gram of FFA in the flask. Add an amount of concentrated sulfuric acid equal to $0.05 \mathrm{~g}$ for every gram of FFA in the flask.

Remember to always add acid to the alcohol and not the other way around. Why?

- Gently agitate the methanol-acid mixture until well-mixed. Add to the feedstock. Agitate at $60-65^{\circ} \mathrm{C}$ for one hour. Let cool.

- Measure the acid value of the fluid.

You might not notice any separation of a methanol-water layer. This can take some time to occur because the densities of the two layers are close.

\section{Reminder: Acid Value Measurement}

1) Prepare two $600 \mathrm{ml}$ beakers by adding $125 \mathrm{ml}$ of solvent. The solvent consists of $50 \%$ isopropyl alcohol $/ 50 \%$ toluene and should be available in the lab.

2) Add about 5 grams of sample (weigh to get exact amount) to one of the beakers.

3) Add $2 \mathrm{ml}$ of phenolphthalein indicator to both beakers.

4) Titrate both beakers with $0.1 \mathrm{~N} \mathrm{KOH}$ to the first permanent pink color in each.

5) Calculate the acid value from: $\mathrm{AV}=(\mathrm{A}-\mathrm{B}) \times \mathrm{N} \times 56.1 / \mathrm{W}$

where $\mathrm{A}=$ number of $\mathrm{ml}$ of $\mathrm{KOH}$ to neutralize sample beaker

$\mathrm{B}=$ number of $\mathrm{ml}$ of $\mathrm{KOH}$ to neutralize blank beaker

$\mathrm{N}=$ normality of $\mathrm{KOH}$ solution ( 0.1 in this case)

$\mathrm{W}=$ weight of sample in grams

Remember that $\% \mathrm{FFA} \approx 1 / 2(\mathrm{AV})$ 


\section{Feedstock Preparation}

It has been mentioned earlier that biodiesel can be produced from a wide variety of feedstocks including vegetable oil, animal fats, restaurant waste oils, and trap grease. Another way to categorize these feedstocks is to identify those coming from oilseeds and those that originate from rendering plants. This section will discuss the processes used to produce these two categories of feedstocks and the implications for biodiesel processing.

\section{Production of oil from oilseeds}

Soybean oil is the largest source of vegetable oil in the United States and rapeseed oil (Canola) is the largest source in Europe. Most of these oils are used as food products but they require considerable processing before they are considered edible. Table 9 shows the composition of crude soybean oil compared with a fully refined oil. Fully refined oils are also known as RBD oils where RBD strands for refined, bleached, and deodorized. Table 9 shows how a crude soybean oil typically contains 1.5 to $2.5 \%$ phosphatides, $1.6 \%$ unsapoifiable matter including 0.15 to $0.21 \%$ tocopherol (Vitamin E), and 0.3 to $0.7 \%$ free fatty acids. These compounds contribute color, odor, and strong flavors to the oil that are usually undesirable for cooking. Some of the compounds also contribute to poor storage stability.

It has been shown that high quality biodiesel can be produced even from crude soybean oil [8]. However, use of partially or fully refined oil simplifies the biodiesel production process so some producers have chosen to use degummed oils or even RBD oils. The following discussion describes current practice for processing soybean oil in the U.S. Similar processes are used for rapeseed oil in Europe.

Table 9. Average Composition for Crude and Refined Soybean Oil [7, p. 14]

\begin{tabular}{|c|c|c|}
\hline & Crude Oil & Refined Oil \\
\hline Triglycerides, $\%$ & $95-97$ & $>99$ \\
\hline Phosphatides, $\%$ a,b & $1.5-2.5$ & $0.003-0.045^{\mathrm{c}}$ \\
\hline Unsaponifiable Matter, $\%$ & 1.6 & 0.3 \\
\hline Plant sterols, $\%{ }^{\mathrm{d}}$ & 0.33 & 0.13 \\
\hline Tocopherols, $\%{ }^{\mathrm{e}}$ & $0.15-0.21$ & $0.11-0.18$ \\
\hline Hydrocarbons, $\%{ }^{\mathrm{f}}$ & 0.014 & 0.01 \\
\hline Free fatty acids, $\%$ & $0.3-0.7$ & $<0.05$ \\
\hline \multicolumn{3}{|l|}{ Trace Metals $^{\mathrm{a}}$} \\
\hline Iron, $\mathrm{ppm}$ & $1-3$ & $0.1-0.3$ \\
\hline Copper, ppm & $0.03-0.05$ & $0.02-0.06$ \\
\hline
\end{tabular}

${ }^{a}$ Evans, C.D., G.R. List, R.E. Beal, L.T. Black. 1974. JAOCS. 51:444-448.

${ }^{\mathrm{b}}$ List, G.R., A.J. Heakin, C.D. Evans, L.T. Black, T.L. Mounts. 1978. JAOCS. 55:521-522.

${ }^{\mathrm{c}}$ Corresponding to $1-15$ ppm of phosphorus

${ }^{\mathrm{d}}$ Weihrauch, J.L., J.M. Gardiner. 1978. J. Am. Diet. Assoc. 73:39-47.

${ }^{\mathrm{e}}$ Bauernfeind, J.C. 1977. Crit. Rev. Food Sci. 8:337-382.

${ }^{\mathrm{f}}$ Gutfinger, T., A. Letan. 1974. J. Sci. Food Agric. 25:1143-1147. 
Oil is removed from soybeans by two techniques: mechanical crushing and solvent extraction. Mechanical crushing is prefered for smaller plants because it requires a smaller investment. Typically plants that process less than $100,000 \mathrm{~kg}$ /day use mechanical crushing and plants that process more than $300,000 \mathrm{~kg} /$ day use solvent extraction [4, p. 113].

Before the oil can be extracted, the seeds must be prepared. This involves removing stems, leaves, stones, sand, dirt, and weed seeds. The stems and stones can be screened out because they are usually larger than the seeds. Weed seeds, sand, and dirt are usually smaller and can be removed by finer screens. Magnets can remove iron particles.

After cleaning, the seeds are often dehulled. The hulls are abrasive and contain very little oil (less than 1\%). Removing the hulls reduces the wear on the screw press. It can also increase the extraction of oil because the hulls absorb oil. On oilseeds with thick shells, dehulling typically involves cracking the shells and then separating the shells by screening or aspiration. With soybeans, dehulling generally includes removal of residual fines.

Oilseeds that are to be processed by solvent extraction are usually flaked to increase the exposure of the oil to the solvent. Hexane in a percolation extractor is the most common technology used today. This type of extractor drips the solvent down on the flaked soybeans so it can dissolve the oil in a manner similar to a coffee percolator. The oil-laden solvent, or miscella, is then filtered and removed from the extractor. To remove the solvent, the miscella is heated to vaporize the solvent. This gets the the miscella to about 5\% solvent [4]. Then the remaining solvent is stripped by injecting steam into the oil. The steam and hexane vapors are condensed and, since the two fluids are insoluble, they can be separated in a settling tank. Figure 1 shows a schematic of a typical solvent extraction plant that includes several stages of solvent recovery.

The alternative to solvent extraction is mechanical extraction or crushing. The beans are generally preheated to destroy enzymes that cause problems with using the meal as animal feed. One popular way of cooking the bean is to use an expanding extruder. This device compresses the beans to very high pressure so that the temperature rises to $150^{\circ}-160^{\circ} \mathrm{C}$, which is high enough to deactivate the enzymes. The extrusion also frees the oil and produces a frothy, liquid that quickly solidifies as the water flashes to vapor and reduces the moisture level from 10-14\% to $6-7 \%$. After cooking or extrusion, the oil seeds are usually fed to a screw press where they are compressed to high pressure. Drainage slots are provided for the oil to leave the press. Moisture control is important to maintain proper temperature. If the moisture level is too high, then beans will be soft and the oil will contain excessive solids. If the moisture level is too low, then the oilseeds will overheat. Ideally, the moisture should be $2-3 \%$ for full pressing and $4-6 \%$ for prepressing [4]. If the beans have not been extruded, then pre-pressing may be used to break the cell walls and release the oil from the seed. It can preceed both mechanical and solvent extraction.

After extrusion from the seed, crude soybean oil contains impurities that can be classified into two categories: oil insoluble and oil soluble [1]. The oil-insoluble impurities consist of seed fragments and meal fines, free water, and long-chain hydrocarbons or waxes that cause cloudiness when the oil is refrigerated. Most of this material can be removed by filtration. As described in Table 1, the oil-soluble materials include free fatty acids, phosphatides, gummy or mucilaginous substances, color bodies, protein, tocopherols, sterols, hydrocarbons, ketones, and 
aldehydes. Some of these compounds, particularly the ketones and aldehydes, can cause the oil to have an unpleasant taste and smell. Other compounds are actually desirable, such as the tocopherols which act as antioxidants. The sterols are relatively inert and can remain in the oil. The food-grade oil typically contains $0.5-1.5 \%$ non-oil compounds that are known collectively as unsaponifiable matter.

Processing of crude soybean oil to food-grade oil generally consists of three steps: refining (degumming and caustic refining), bleaching, and deodorization. As mentioned earlier, these oils are known as RBD oils (refined, bleanched, and deodorized).

\section{Refining}

The refining step is designed to remove the phospholipids and free fatty acids from the crude oil. Crude soybean oil typically contains $500-700$ ppm of phosphorus which corresponds to $1.5 \%$ to $2.1 \%$ phospholipids [1]. The main reason to remove these phospholipids is that some of the compounds, particularly the calcium and magnesium salts of phosphatidic and lysophatidic acids, are strong emulsifiers. If these compounds are still present during the later alkali neutralization step, they will inhibit the separation of the soaps and lower the yield of neutral oil. Phospholipids (also called phosphotides) will also react with water to form insoluble sediments that are not desirable when cooking with the oil.

Refining may consist of two steps. The first step is degumming. In this step, crude soybean oil is mixed with $1 \%-3 \%$ of water and the mixture is agitated mechanically for 30-60 minutes at $70^{\circ}$ $\mathrm{C}\left(158^{\circ} \mathrm{F}\right)$. This hydrates the phospholipids and gums and these hydrates are insoluble in the oil. They can be separated by settling, filtering, or centrifuging. The phosphorus content can be lowered to $12-170 \mathrm{ppm}$ by this procedure. The byproduct of water degumming has value as a feedstock for lecithin production.

Table 1 shows the profile of phospholipids from lecithins produced from various oilseeds. It shows that there are significant differences in the distribution of compounds between the different feedstocks.

A portion of the phosphatides are not hydratable by contact with water alone. The addition of citric or phosphoric acid will hydrate those remaining. Citric acid is prefered if the byproduct is to be used for lecithin. The acid hydration is accomplished by adding $0.05-0.2 \mathrm{wt} \%$ of concentrated (85\%) phosphoric acid to crude oil, which has been heated to $60-85 \mathrm{C}$. The residence time varies from a few seconds to 1-2 minutes depending on the type and quality of the oil. The extent of gum removal by these techniques is related to the strength of the acid treatment. Figure 29 shows a schematic diagram of the processes involved to degum soybean oil and produce edible lecithin. 


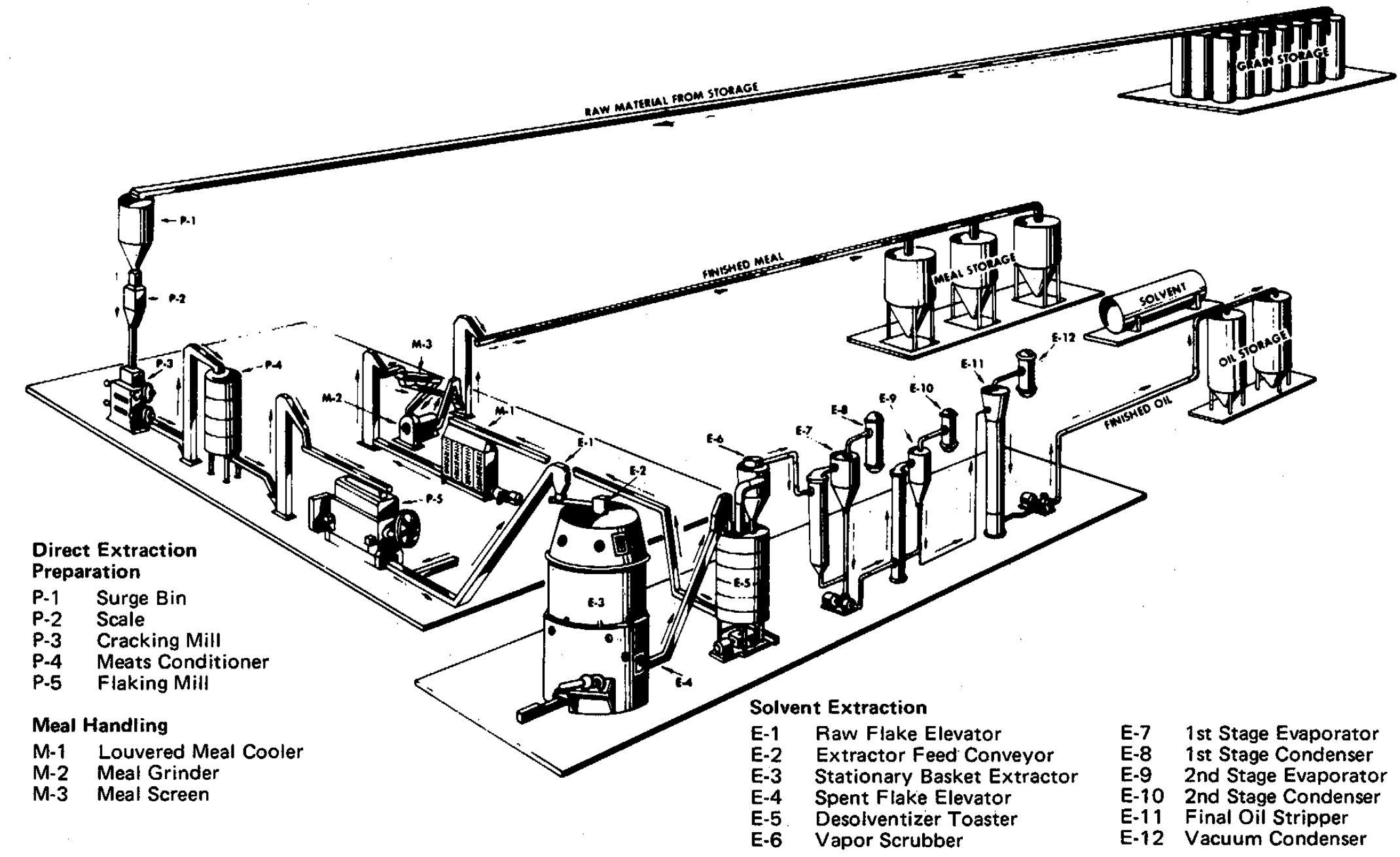

Figure 28. Soybean oil extraction plant [7]. 
Table 10. Phospholipid Composition [wt\%] of Commercial Lecithins [2, p. 52]

\begin{tabular}{c|c|c|c}
\hline Phospholipid & Soy & Rapeseed & Sunflower seed \\
\hline Phosphatidylcholine & 21.9 & 24.6 & 25.4 \\
Phosphatidylethanolamine & 13.6 & 22.1 & 11 \\
phosphatidylserine & & & 0.8 \\
phosphatidylinositol & 12 & 14.7 & 19.4 \\
PG+ DPG* & 2.3 & & 1.2 \\
Phosphatidic Acid & 5.8 & & 3.3 \\
N-Acyl-phosphatidylethano & 2.8 & & 1 \\
lamine & 2.9 & 19.4 & \\
Lyso-phospholipids & 3.6 & 19.2 & \\
Others & & & \\
\hline
\end{tabular}

* PG + DPG = Phosphatidylglycerol and Diphosphatidylglycerol. Note: some commercial lecithins contain glycolipids (phosphorus-free polar lipids) that are not included in the totals given in the table.

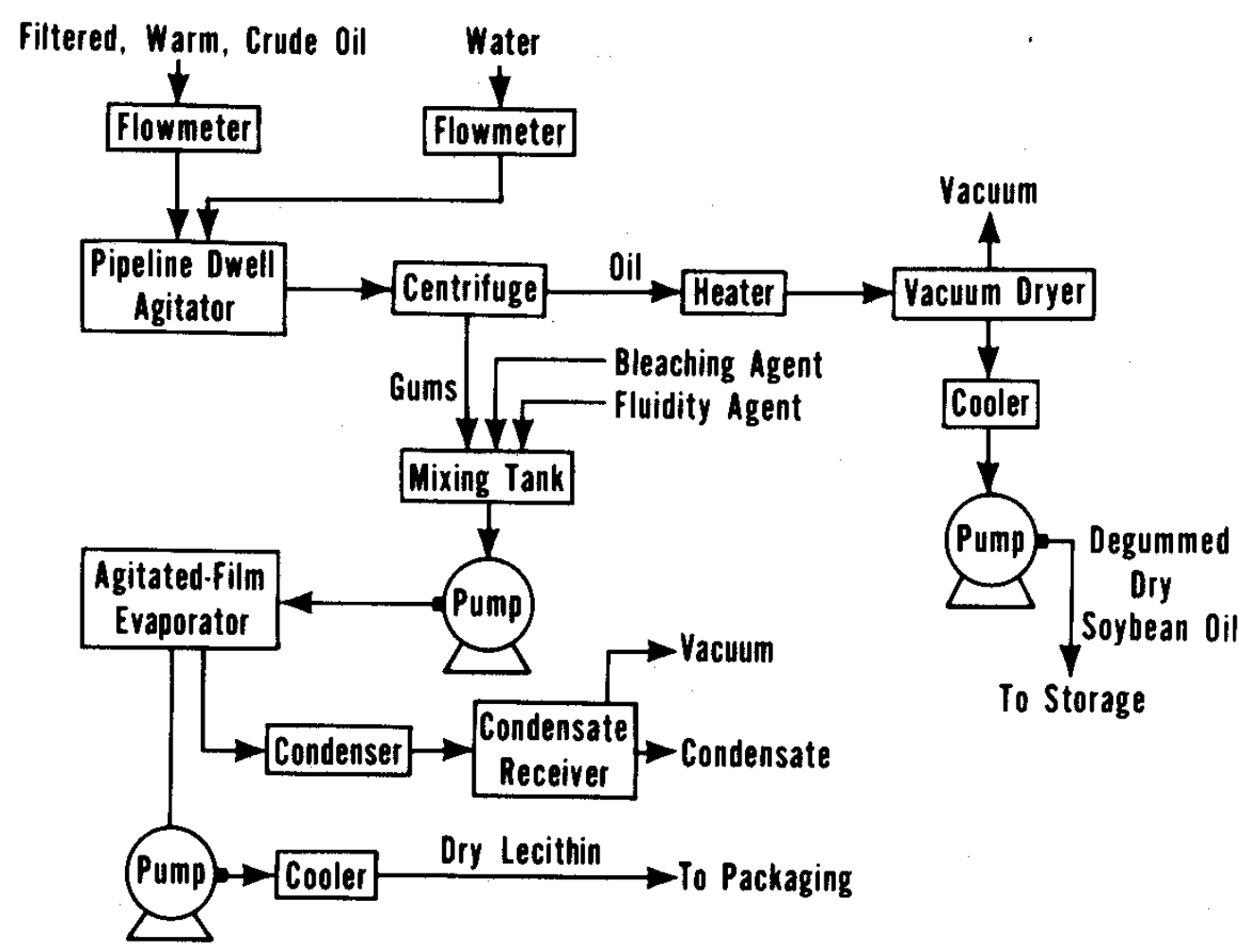

Figure 29. Flow diagram for soybean oil degumming [7].

The second phase of refining is neutralization, or caustic refining. This process is to remove the free fatty acids present in the crude oil. An alkali solution, usually sodium hydroxide, is added that reacts with the free fatty acids to produce soaps. The soaps are insoluble in the oil and easily 
separated by water washing. The alkali solution also neutralizes any acid remaining from the degumming stage.

The alkali will react with the triglycerides in the oil also, so the neutralization parameters (type or alkali, solution strength, temperature, agitation, and time) must be optimized to minimize the yield loss. There can be additional losses from emulsification and suspension of oil droplets in the soap solution. A byproduct of caustic refining is a mixture of soap, water and oil known as soapstock. This has been considered as a feedstock for biodiesel as its cost is low, but its high water content and converting the soaps to methyl esters are significant obstacles to cost-effective utilization. Figure 30 shows a diagram of the caustic refining process.

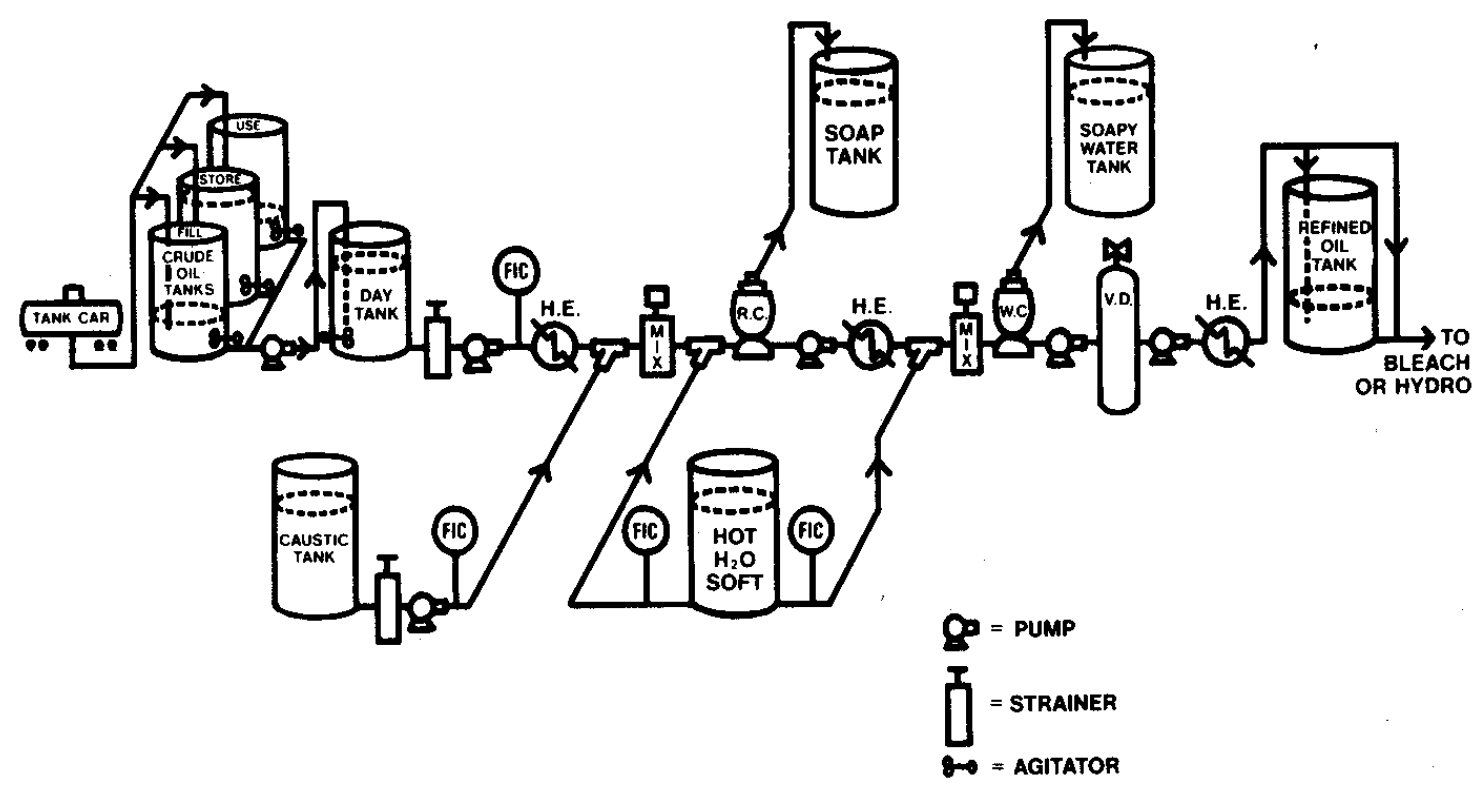

Figure 30. Flow Diagram for Caustic Refining of Soybean Oil [7].

\section{Bleaching}

The primary purpose of bleaching is to remove the color pigments from the oil. It also helps to remove remaining soap, trace metals, phosphatides, and sulfur compounds. Hydroperoxides, the initial products of oxidation are broken down during the bleaching process and some of the final oxidation products, the carbonyl compounds, are removed.

Bleaching involves mixing bleaching clays, consisting of natually occurring bentonite and montmorillonite clays, with the oil and agitating for 10-30 minutes. The oil is usually heated to $90^{\circ}-120^{\circ} \mathrm{C}$ and the process occurs under a slight vacuum to exclude oxygen. A consequence of the vacuum and high temperature is that residual water from the neutralization and water washing is removed. This is not always desirable because the effectiveness of the clays is usually enhanced by the presence of a small amount of water. The clays are usually activated before use 
with a mineral acid such as sulfuric acid. This removes some of the minerals from the clay and produces a larger volume of micropores and smaller clay particles.

The bleaching clays are mixed with the oil, either directly or by premixing with a small amount of oil to produce a slurry and then adding the slurry to the oil. After the adsorption processes have reached equilibrium, the clay is filtered out by self-cleaning filters.

The peroxide value of the oil is very low after bleaching because the hydroperoxides have been eliminated but the free fatty acid level may be slightly higher. This may be due to some of the sulfuric acid leaching out of the clay or to hydrolysis of the oil. Hydrolysis is a chemical reaction between the oil and water that produces free fatty acids and glycerin.

\section{Deodorization}

Deodorization is the final processing stage before the oil is ready for use in food products. The deodorization removes the trace components that give the oil an unpleasant taste and odor.

Deodorization is essentially a distillation process that occurs at high temperature $\left(200^{\circ}-260^{\circ} \mathrm{C}\right)$ and low pressure (2 - 7 torr, 2.5-9.2 mbar). The odor causing compounds are more volatile than the oil and will be removed by extended heating at high temperature and low pressure.

The first step of deodorization is deaeration. This step removes all the dissolved oxygen so that the oil does not oxidize at the high temperatures used in deodorization. In batch deodorizers, the deaeration occurs naturally while the oil is heated from the typical inlet temperature of $40^{\circ}-80^{\circ}$ $\mathrm{C}$ to the deodorization temperature range of $200^{\circ}-260^{\circ} \mathrm{C}$. If adequate vacuum is available, the oil should be deaerated by the time the oil reaches $100^{\circ}-120^{\circ} \mathrm{C}$.

Preheating is sometimes identified as the second step of deodorization. In continuous flow deodorization processes, a portion of the heat required for heating the oil comes from cooling the deodorized oil. As much heat as possible is transfered from the final product to incoming oil. After preheating, the oil is heated to its final temperature by steam or another heating fluid such as Dowtherm A.

After the oil has been held at high temperature for sufficient time $(2-5$ hours in a batch deodorizer), it is cooled to $120^{\circ} \mathrm{C}$ where it is common practice to add $50-100 \mathrm{mg} / \mathrm{kg}$ of citric acid. The citric acid is added to chelate trace metals in the oil so that they can be removed by filtration. Chelation is a chemical process where a compound, known as a chelating agent, binds to a metal compound and makes it unavailable for further reaction. Then the oil is cooled further to its storage or bottling temperature. One final stage of filtration, known as polish filtration, occurs immediately before the oil leaves the plant.

As mentioned earlier, biodiesel can be produced from oil at any stage in the various processes. The compounds removed during refining, bleaching, and deodorization are mostly removed by water washing or collect in the glycerol phase. Biodiesel produced from crude soybean oil retains somewhat more color than biodiesel from refined oil but phosphorus and free fatty acid levels seem to be equivalent [8]. 
Table 11. American Fats and Oils Association Specifications for Tallows and Greases*

\begin{tabular}{|c|c|c|c|c|c|}
\hline Grades & $\begin{array}{l}\text { Titre, } \min \\
{ }^{\circ} \mathrm{C}\end{array}$ & FFA, max & FAC, color, $\max$ & $\mathrm{R} \& \mathrm{~B}, \max$ & $\mathrm{MIU}$ \\
\hline Edible tallow & 41.0 & 0.75 & 3 & None & $* * * *$ \\
\hline Lard (edible) & 38.0 & 0.50 & $* *$ & None & $* * * *$ \\
\hline Top white tallow & 41.0 & 2 & 5 & 0.5 & 1 \\
\hline $\begin{array}{l}\text { All beef packer } \\
\text { tallow }\end{array}$ & 42.0 & 2 & None & 0.5 & 1 \\
\hline Extra fancy tallow & 41.0 & 3 & 5 & None & 1 \\
\hline Fancy tallow & 40.5 & 4 & 7 & None & 1 \\
\hline $\begin{array}{l}\text { Bleachable fancy } \\
\text { tallow }\end{array}$ & 40.5 & 4 & None & 1.5 & 1 \\
\hline Prime tallow & 40.5 & 6 & 13-11B & None & 1 \\
\hline Special tallow & 40.0 & 10 & 21 & None & 1 \\
\hline No. 2 tallow & 40.0 & 35 & None & None & 2 \\
\hline A tallow & 36.0 & 15 & 39 & None & 2 \\
\hline $\begin{array}{l}\text { Choice white } \\
\text { grease }\end{array}$ & 36.0 & 4 & $13-11 \mathrm{~B}$ & None & 1 \\
\hline Yellow grease & $* * *$ & 15 & 39 & None & 2 \\
\hline
\end{tabular}

* from the National Renderers Association

** Lovibond color 5-1/4 inch cell - max 1.5 red. Lard peroxide value 4.0 ME/K max.

*** Titre minimum, when required, to be negotiated between buyer and seller on a contract basis.

**** Moisture maximum $0.20 \%$. Insoluble impurities maximum $0.05 \%$.

\section{Rendering}

Rendering is the process whereby by-products of the food industry, including animal fat, bone, offal, hides, feathers, and blood are recycled into usable products. In the case of rendering operations that may be attached to slaughtering facilities, the products may be edible fats and proteins that are suitable for human consumption. These edible fats may be refined, bleached and deodorized in a manner similar to the described above for vegetable oils. This provides a product that is suitable for food, cosmetics, and other high value uses. Table 11 shows the specifications for the wide variety of edible and inedible tallows and greases produced from rendered fats.

Larger volumes of material are processed in plants that produce so-called "inedible" products, although the products are suitable for use in animal feeds. These inedible rendering plants may process fat and bone trimmings, meat scraps, restaurant grease, fallen animals, and other sources of fat and protein. Table 12 shows the composition and tallow and grease yield of various raw materials for inedible rendered products.

Figure 31 shows a typical rendering operation. Dead stock carcasses and other raw materials are received by the plant and fed in a continuous flow to a crusher that grinds the material to a uniform paste-like consistency. 
Table 12. Composition of Raw Materials for Inedible Rendering Products*

\begin{tabular}{|l|c|c|c|}
\hline \multicolumn{1}{|c|}{ Source } & $\begin{array}{c}\text { Tallow/grease } \\
\text { wt\% }\end{array}$ & $\begin{array}{c}\text { Protein solids } \\
\text { wt\% }\end{array}$ & $\begin{array}{c}\text { Moisture } \\
\text { wt\% }\end{array}$ \\
\hline Packing house offal and bone & \multicolumn{2}{|c|}{} \\
\hline Steers & $30-35$ & $15-20$ & $45-55$ \\
\hline Cows & $10-20$ & $20-30$ & $50-70$ \\
\hline Calves & $10-15$ & $15-20$ & $65-75$ \\
\hline Sheep & $25-30$ & $20-25$ & $45-55$ \\
\hline Hogs & $25-30$ & $10-15$ & $55-65$ \\
\hline Dead stock (whole animals) & \multicolumn{5}{|c|}{} \\
\hline Cattle & 12 & 25 & 63 \\
\hline Calves & 10 & 22 & 68 \\
\hline Sheep & 22 & 25 & 53 \\
\hline Hogs & 30 & 28 & 32 \\
\hline $\begin{array}{l}\text { Butcher shop fat and } \\
\text { bone }\end{array}$ & 31 & 32 & $82-84$ \\
\hline Blood & None & $16-18$ & 25 \\
\hline Restaurant grease & 65 & 10 & 65 \\
\hline Poultry offal & 10 & 33 & 67 \\
\hline Poultry feathers & none & 25 & \\
\hline
\end{tabular}

* from National Renderers Association

This material is then fed to a cooker that heats the material to $121-135^{\circ} \mathrm{C}\left(250-275^{\circ} \mathrm{F}\right)$. The high temperature encourages the removal of water and separates a portion of the fat from the solids. The fat and solids are fed to a drain pan where the fat is drained off and the solids are fed to a screw press where the remaining fat can be squeezed out. The dry solids are ground to a fine powder and sold as meat and bone meal. The fat is centrifuged or filtered to remove particulates. Since the fat may still contain some water, it is common to reheat the fat with steam injection to encourage separation of the water. After heating, the dry fat will rise to the top, the water will settle to the bottom and an interphase layer will appear that consists of emulsified water and oil and possibly protein and minerals. This material is separated and returned to the cooker for reprocessing.

Inedible fats with free fatty acid levels below 15\% and MIU (moisture, insolubles, and unsaponifiables) less than $2 \%$ are generally sold as yellow grease. Fats with higher free fatty acid levels are sold as brown grease. Although their products are referred to as inedible, most rendered material is reintroduced to the food chain as animal feed. Meat and bone meal are used to add protein and minerals to feed and grease is used to increase calories or energy content. Renderers are coming under increasing scrutiny because of concerns about disease transmission. Most renderers will no longer accept sheep carcasses in the United States because of concerns about scrapies. The same concern also exists with deer and elk carcasses, due to chronic wasting disease, a relative of Bovine 


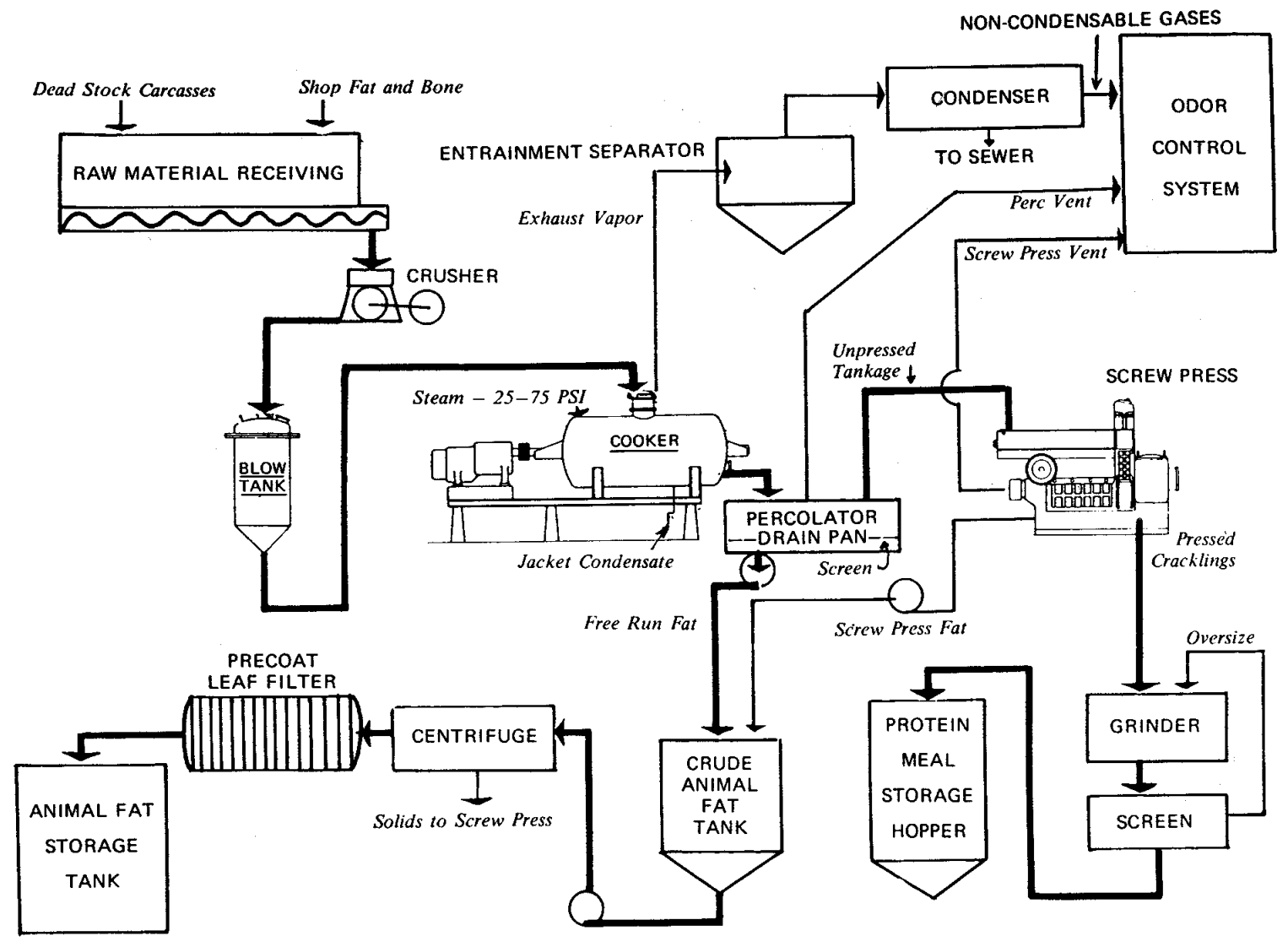

Figure 31. Rendering plant operations

Spongiform Encephalapathy (BSE - Mad Cow Disease). Products from ruminants(cattle) are not fed to other cattle. This requirement has been reinforced by recent events in England where BSE has spread to large herds of cattle through use of infected animal products in the feed. The risks of disease transmission are thought to be minimal in the United States and use of animal products across species is generally considered safe. However, concerns about BSE may cause future legislation further restricting the use of animal byproducts in animal feed and if this occurs, biodiesel may become the most significant market for animal fat.

\section{References}

1. Hui, Y.H., Editor, Bailey's Industrial Oil and Fat Products, V. 2. Edible Oil and Fat Products: Oils and Oilseeds, Chapter 11 Soybean Oil, Fifth Edition, Wiley-Interscience, New York.

2. Schneider, M., "Phospholipids," Chapter 3 from Lipid Technologies and Applications, Edited by F.D. Gunstone and F.B. Padley, Marcel Dekker, New York, 1997. 
3. Allen, D.A., "Refining," Chapter 6 from Lipid Technologies and Applications, Edited by F.D. Gunstone and F.B. Padley, Marcel Dekker, New York, 1997.

4. Williams, M.A., "Extraction of Lipids from Natural Sources," Chapter 5 from Lipid Technologies and Applications, Edited by F.D. Gunstone and F.B. Padley, Marcel Dekker, New York, 1997.

5. Peterson, C.L., M. Feldman, R. Korus, and D.L. Auld, "Batch Type Transesterification Process for Winter Rape Oil,” Applied Engineering in Agriculture, Vol. 7, No. 6, Nov. 1991, pp. 711-716.

6. Peters, Richard, Process Chemical Engineer for Proctor and Gamble, Personal Communication.

7. Erickson, D.R., E. H. Pryde, O.L. Brekke, T.L. Mounts, and R.A. Falb, Editors, "Handbook of Soy Oil Processing and Utilization," Published jointly by the American Soybeasn Association, St. Louis, MO, and the American Oil Chemists" Society, Champaign, IL, 1980.

8. Van Gerpen, J.H. and B. Dvorak, "The Effect of Phosphorus Level on the Total Glycerol and Reaction Yield of Biodiesel," presented at Bioenergy 2002, The $10^{\text {th }}$ Biennieal Bioenergy Conference, Boise, ID, Sept. 22-26, 2002.

9. Franco, D.A. and W. Swanson, The Original Recyclers, Animal Protein Producers Industry, 1996. 


\section{Feedstock Quality Issues}

As a general rule, the closer a feedstock is to containing pure triglycerides, the easier it will be to convert it to biodiesel, and the higher will be its cost. The lower the cost of the feedstock, the more difficult and more expensive it is to convert to biodiesel.

Biodiesel feedstocks will contain many possible contaminants such as water, free fatty acids, particles, and phospholipids. There is potential for each of these contaminants to impact the quality of the final biodiesel product.

\section{Water}

It is important that water be kept out of the biodiesel production process. While most processes can tolerate up to $1 \%$ water, even this low level will increase soap production and measurably affect the completeness of the transesterification reaction. Water can be removed by several techniques. Heating to break any emulsions between the water and oil and then allowing the water to settle to the bottom can remove water. Centrifuges can also be used to separate water and oil. Heating the oil under pressure and then spraying into a vacuum chamber can bring the water content to a very low level.

Crude soybean oil typically contains about $0.3 \%$ water. This can be removed easily by a flash vaporization process. Yellow grease (and usually brown grease) will contain about $0.5 \%$ water because it has been dewatered during previous processing steps, although the sum of the water, insolubles, and unsaponifiables (MIU) is allowed to be as high as $2 \%$. Restaurant grease and trap grease that have not been rendered should always be checked for water content because it may be very high.

Water not only impacts the transesterification process, it can impact the effective cost of the feedstock. Analyses of two actual samples of trap grease are shown below.

\begin{tabular}{lll} 
& \multicolumn{1}{l}{ Sample A } & Sample B \\
Water & $81.5 \%$ & $40.5 \%$ \\
Solids & $6.34 \%$ & $10.2 \%$ \\
Lipids & $12.2 \%$ & $49.3 \%$
\end{tabular}

Trap grease is a very inexpensive feedstock. In most cases, restaurants will pay to have the material removed. However, even if the cost to have the material brought to the plant were as low as 2 cents/lb, the actual cost for the lipid material in sample A would be more than 8 times higher, or over 16 cents/lb. This is a pretty high price for material that is almost all free fatty acids. This price also does not include the added cost of disposing of the water after it is removed or the cost of landfilling the solids.

\section{Solids}

Feedstocks used for biodiesel should always be filtered to ensure no particles enter the processing scheme. This can be a particular problem with recycled products such as trap grease, 
which typically contains fine particles that may require a fine filter. A $100 \mu \mathrm{m}$ filter will suffice to remove particles from most feedstocks although all fuel leaving the plant should be filtered to $5 \mu \mathrm{m}$.

\section{Free Fatty Acids}

The impact of free fatty acids (FFAs) on the biodiesel production process have been discussed extensively elsewhere. While FFAs can impact the production process by deactivating the catalyst, creating soap, and by releasing water when they are converted to esters, they do not seem to impact the final acid value of the biodiesel. Biodiesel produced using a normal alkalicatalyzed process that meets the ASTM specification for total glycerol will usually have 0.2$0.3 \%$ FFAs regardless of the initial acid content of the feedstock. The alkali catalyst also serves as a caustic stripper and removes the FFAs by converting them to soap that is removed during washing.

\section{Phosphorus}

Crude soybean oil contains from 600-900 ppm of phosphorus in the form of phospholipids or gums. This material must be removed because the ASTM specification from biodiesel limits the phosphorus content to $10 \mathrm{ppm}$. Fortunately, even if the oil is not degummed to remove this material, the usual biodiesel production process removes the phosphorus and it is either transferred to the glycerol fraction or removed by water washing. There does not seem to be any effect of the initial phosphorus content of the feedstock on the final phosphorus content of the biodiesel.

\section{Sulfur}

One of the frequently-touted advantages of biodiesel is that it contains very low levels of sulfur. The Environmental Protection Agency has mandated that sulfur levels for petroleum-based diesel fuel must drop to 15 ppm from 500 ppm by 2006. Most biodiesel is already below this level. However, some samples of biodiesel from yellow grease and animal fat have shown sulfur levels as high as 40-50 ppm. The source of this sulfur is still not completely understood, but it may be originating from food preservatives, proteins, the hair on the animal hides and in those cases where poultry are being processed, from chicken feet. Additional research is needed in this area.

\section{Iodine Value}

The iodine value is a very crude but commonly used indicator of the level of saturation of an oil. While saturation and fatty acid profile do not seem to have much impact on the transesterification process, they do affect the properties of the biodiesel. Saturated fats produce a biodiesel fuel with superior oxidative stability, a higher cetane number, but poor low temperature properties. Biodiesel from saturated fats is more likely to gel at ambient temperatures than biodiesel from vegetable oils, especially soybean oil. 


\section{Plant Safety}

\section{Introduction}

The responsibility for the safe operation of any chemical process facility, including a biodiesel plant, begins with the CEO of the company and extends to every employee and visitor on the plant site. Safety is a result of training, operating, maintaining, and preparing to prevent unplanned and dangerous events in the plant. It is a way of thinking and acting during routine activities and in response to extraordinary situations.

There are numerous regulatory requirements associated with training and housekeeping to monitor and improve safety in the workplace. Regulations and procedures dictated by OSHA, EPA, and other agencies apply to biodiesel plants of any size. Some of these requirements are covered in other modules. In addition, every plant should have one person with the designated responsibility for overseeing the training and inspections in the workplace.

Given this introduction, this module will focus on some of the key elements of a plant safety program, safe design, and safe operation. The emphasis is on elements of safety associated with the operation of a chemical process facility. This module is intended to be a reminder for what must be known and taught, more than detailed instruction in all of the elements of plant safety.

\section{Basic Definitions}

-Safety: The prevention of accidents by the use of appropriate technologies to identify the hazards of a chemical plant and to eliminate them before the accident occurs.

-Hazard: Anything with the potential for producing an accident.

-Risk: The probability of a hazard resulting in an accident.

\section{Keys to a Successful Safety Program}

-Safety Knowledge - Every person associated with the plant must know what the materials used in the plant are and what their hazards are. The people must be trained in the correct and safe operation of the processing equipment, and know how to respond in an emergency situation.

-Safety Experience - Training is more than a lecture, a video module, or reading a manual. Personnel must have hands-on training in proper operation of equipment and develop an understanding of how to recognize and correct dangerous situations. The use of process simulators is needed, as is training with actual safety equipment.

-Technical Competence - The ability to use training in proper operation and proper safety precautions must be demonstrated and documented on a regular basis. 
-Safety Management Support - Safety must be modeled and monitored throughout the management chain.

-Commitment - Demonstrated commitment to safety in the workplace and beyond must begin with the CEO and permeate all employees. Breaches of safe procedures must be clear dismissal grounds and enforced.

\section{Types of Chemical Plant Accidents}

A biodiesel facility is first and foremost a chemical process facility. As such, the plant is subject to the same dangers as any other chemical process plant. Table 13 lists categories of accidents that are uniquely associated with chemical plants. Note that the likelihood of an event and its potential impacts are not closely related.

Table 13. Types of Chemical Plant Accidents

\begin{tabular}{|l|l|l|l|}
\hline & Probability & $\begin{array}{l}\text { Potential } \\
\text { Fatalities }\end{array}$ & Potential \$ Loss \\
\hline Fire & High & Low & Moderate \\
\hline Explosion & Moderate & Moderate & High \\
\hline Toxic Release & Low & High & Low \\
& & & \\
\hline
\end{tabular}

For a biodiesel plant, the greatest risk is associated with methanol. It presents a fire and explosion hazard as well as being toxic through oral ingestions of skin contact.

In order to better understand how accidents occur in a chemical plant, it is useful to examine the causes for loss with respect to the source of the loss event. These causes are listed in order of frequency in Table 14. 


\title{
Table 14. Causes for Loss in a Chemical Plant Accident
}

(in order)

\author{
-Mechanical Failure \\ -Operational Error \\ -Unknown - Miscellaneous \\ -Process Upset \\ - Natural Hazard \\ -Design Error \\ -Arson - Sabotage
}

Since the most common cause for loss is mechanical failure, it is useful to examine the specific equipment items responsible for the failures. These items are listed in order in Table 15.

\section{Table 15. Hardware Associated with Losses}

-Piping Systems
-Miscellaneous of Unknown
-Storage Tanks
-Reactor Piping Systems
-Process Holding Tanks
-Heat Exchangers
-Process Towers
-Compressors
-Pumps
- Gages

\section{Accident Sequence}

It is rare that there is a single causal event that leads to a loss accident. Indeed, one of the most important ways to prevent accidents is to examine carefully the chain of events that result in an accident and that bring the accident sequence to an end. These lesson's learned provide the basis for anticipating potential accident situations and planning interventions that halt the accident process before a loss occurs. The accident sequence can be described as follows:

-Initiation - event that begins the accident

-Propagation - event(s) that sustain or expand the accident

-Termination - event(s) that stop the accident or diminish it is size

The propagation steps in an accident sequence are generally associated with potential hazards that exist - accidents ready to happen. In many cases these steps are the results of intentional acts with unintended consequences. For example, turning off alarms and sensors during servicing makes the service personnel vulnerable to accidents that would have been prevented if the alarms were operational. The chemical process industry has a special category of hazards present 
because the industry is based on the utilization and transformation of chemical raw materials into new chemical products. These hazards are linked to the physical and chemical reactivity properties of the various chemicals present. The categories of chemical process hazards the may be present in a chemical facility are listed in Table 16.

\section{Table 16. Categories of Chemical Process Hazards}

-Explosion

-Gases - toxic, flammable, compressed

-Flammable Liquids

-Flammable Solids

- Oxidizers and Organic Peroxides

-Poisons, Pesticides, Carcinogens

-Radioactives

- Corrosives

\section{Identifying Hazards}

The most fundamental tasks in developing a safety program for a chemical process facility are summarized in the sequence shown below.

-What are the hazards? - Identification

-What can go wrong and how? - Scenario Building

-What are the chances? - Risk

-What are the consequences? - Results

There are a number of formalized procedural tools for hazard identification. Any given facility may choose to use one or more of these tools. The tools include:

- Process Hazards Checklist - Use a standard checklist that covers possible hazardous conditions in the operation. When a hazard is noted, the action step is to remove the hazard, or to mitigate its effect.

- Hazard Survey - Use an individual or a team to inspect a specific process or operational area, looking for hazardous conditions. Again, the action step is to eliminate or mitigate the hazard.

- Hazard and Operability Studies HAZOP - This is a formalized approach that examines every piece of equipment in a process on the basis of its function. The questions of cause and effect for each operation are framed in terms of the item not functioning, under functioning and over functioning (NOT, LESS, MORE, etc.). The effect of each condition is estimated, along with the likelihood of the cause being present. This process helps identify likely, significant faults for correction. 
- Safety Review - An independent, experienced team reviews operations and safety procedures in use at a facility. The review includes simulated incidents and monitors the response to the incidents.

\title{
Toxic Hazards
}

In the chemical process industry there is the hazard of exposure to chemicals that are toxic. Toxicity is defined as the ability of a chemical substance to cause physical harm to a living organism. The harm may range from a temporary irritation to permanent loss of function, to death. The toxic effect may be the result of a long term, cumulative effect (chronic toxicity) or a rapid response to the presence of the toxicant (acute toxicity).

In either case, a key factor in the response is the amount of material presented in the exposure (dose) and the degree of toxicity exhibited by the material. The dose is typically measured in terms of amount per kg of body weight. The degrees of toxicity based upon the dosage required to cause death in 50 percent of the exposed subjects is shown in Table 17.

\section{Table 17. Degree of Toxicity $\mathrm{LD}_{50}$}

\section{(dose per kg body weight)}

$$
\begin{aligned}
& \cdot<1.0 \mathrm{mg} \\
& \cdot 1.0-50 \mathrm{mg} \\
& \cdot 50-500 \mathrm{mg} \\
& \cdot 0.5-5 \mathrm{gm} \\
& \cdot 5-15 \mathrm{gm} \\
& \cdot>15 \mathrm{gm}
\end{aligned}
$$

\author{
Dangerously toxic \\ Seriously toxic \\ Highly toxic \\ Moderately toxic \\ Slightly toxic \\ Extremely low toxicity
}

When working with and around various chemicals, there are a number of paths for toxic materials to enter the body. These routes include:

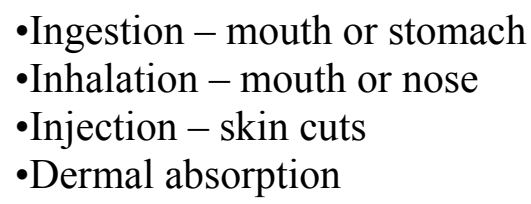

The toxic hazards can be eliminated from the body by excretion (kidneys, liver, lungs, other), detoxification through internal biotransformation, or by storage, typically in fatty tissue or bones. Methanol is the primary issue for toxic exposure in a biodiesel plant.

Because of the risks from chronic exposure, there are limits to allowable exposures to most chemicals. These limits are based on a combination of time and concentration. these limits are termed "threshold limit values" and are described below. 


\section{Threshold Limit Values}

-TLV-TWA: Time weighted average, $8 \mathrm{hr}$ Day

-TLV-STEL: Time weighted average, short-term exposure, $<15 \mathrm{~min}$

-TLV-C: Ceiling limit, do not exceed at all

A required source of information on the hazards associated with chemicals in use in the workplace is the Material Safety Data Sheet. There must be copies of the MSDS for every chemical in use in each area of the chemical plant and associated laboratories available in a public, easily located place. The hazard properties and recommended methods of handling each compound are included in the MSDS. All employees must be familiar with the chemicals that they are working with and must follow the recommendations for their handling, use and disposal.

\section{Designing for Safety}

There are a number of safety enhancement strategies -that can be applied during the design phase for any chemical plant. They include:

-Substitution - Less hazardous chemicals and equipment

-Attenuation - Less hazardous operating conditions = "less"

-Isolation - Isolate equipment from sources of hazard or from risk of harm to personnel

-Process intensification - smaller/higher throughput; smaller inventory

Similarly, there are safety enhancement strategies to be applied during the operating phase of a chemical plant:

-Enclosures

-Local Ventilation

-Dilution Ventilation

-Wet Methods and Cleaning

-Good Housekeeping

-Personal Protection

\section{Summary}

-Safety is PREPARED

-Safety is PREPLANNED

-Safety is PREVENTION

-Safety is PREEMINENT 


\section{Biodiesel Transportation and Storage}

There are several key properties that need to be considered in the transportation and storage of biodiesel. These properties include exposure temperature, oxidative stability, fuel solvency, and material compatibility. Exposure temperature refers to the temperature at which the biodiesel is being stored or transported. Oxidative stability concerns the ability of the biodiesel to be stored for extended periods without degradation. Fuel solvency refers to the solvent properties of the biodiesel. The interaction of the biodiesel with materials of construction for storage tanks, seals, and gaskets is the material compatibility. Each of these properties will be discussed in more detail and within the context of storage.

All types of diesel fuels can gel at low temperatures. The temperature required to induce gelling (freezing) in biodiesel is generally higher than that of conventional diesel fuel and depends on the composition of the biodiesel. In particular, saturated methyl esters will have higher freezing points than unsaturated methyl esters. The composition of the biodiesel is, of course, dictated by the oil feedstock used for its production.

As with gelling, all diesel fuels have the potential for oxidative stability issues. Oxidative stability concerns the degradation of diesel fuel due to reaction of the fuel with oxygen and catalysts. As such, the reactivity of the fuel is important. This reactivity can be related to the presence of $\mathrm{C}=\mathrm{C}$ (olefinic) bonds in the fuel with increased content of the $\mathrm{C}=\mathrm{C}$ bonds correlated to decreased oxidative stability of the diesel fuel. The increase in instability of a given diesel fuel molecule is generally directly proportional to the increase in the number of $\mathrm{C}=\mathrm{C}$ bonds in the molecule (i.e., a molecule containing two $\mathrm{C}=\mathrm{C}$ bonds has half the stability of a molecule containing one $\mathrm{C}=\mathrm{C}$ bond). The oxidative stability of a diesel fuel can be estimated using the iodine number (ASTM D 1510). The longer term stability of a diesel fuel can be evaluated using an accelerated stability test (ASTM D 2274). Oxidative degradation of diesel fuel is manifest by higher acid numbers, increased viscosity, and the formation of gums and sediments.

While biodiesel is only a mild solvent, it does have higher solvency properties than diesel fuel. Due to this property, residual sediments in diesel storage tanks or vehicle fuel tanks can be solvated by biodiesel. The solvent properties of biodiesel are mitigated by the use of biodiesel in blends with diesel fuel. Typically, $20 \%$ or less blends of biodiesel in diesel will nearly completely dilute the solvency effect. However, this solvency effect should be considered if pure biodiesel is stored in a tank that was previously used for standard diesel.

The material compatibility is also an important property to consider with biodiesel. It is best to use stainless steel or aluminum equipment in the processing of biodiesel. Oxidation and sediment production in either biodiesel or diesel can be initiated by contact with brass, bronze, copper, lead, tin and zinc. Biodiesel can have compatibility issues with some polymers. Shown in Table 18 is compatibility of biodiesel with a number of polymers relative to the polymer's compatibility with standard diesel. Biodiesel is compatible with the seals, gaskets and adhesives commonly used today. However, there may be issues with pre-1993 seals, gaskets and adhesives. 


\section{Table 18: Biodiesel material compatibility.}

\begin{tabular}{ll} 
Polymer & Relative to Standard Diesel \\
\hline Fluorosilicon & Hardness little change, swell $+7 \%$ \\
Nitrile & Hardness $-20 \%$, swell $+18 \%$ \\
Nylon 6/6 & Little Change \\
Polypropylene & Hardness $-10 \%$, swell $+8-15 \%$ \\
Polyurethane & Hardness little change, swell $+6 \%$ \\
Polyvinyl & Much worse \\
Teflon & Little change \\
Tygon & Worse \\
Viton A401-C & Little change \\
Viton GFLT & Little change
\end{tabular}

When storing biodiesel, the temperature that is experienced by the biodiesel must be closely monitored so as to avoid the formation of crystals. These crystals are formed by the solidification (freezing) of the biodiesel. Crystal formation is undesirable since the crystals can plug fuel lines and fuel filters. To avoid the formation of crystals, biodiesel should be stored at a temperature at least $15^{\circ} \mathrm{F}$ higher than the pour point of the fuel. For most pure biodiesel storage temperatures of $45-50^{\circ} \mathrm{F}$ are generally adequate. Even in most cold climates, underground storage of pure biodiesel commonly provides the necessary storage temperature to prevent crystal formation. If pure biodiesel is stored above ground in cold climates, the storage system needs to include additional features such as insulation, agitation capability, and/or heating capability. Blends of biodiesel with standard diesel can frequently be stored at lower temperatures. These blends still need to be stored at least $15^{\circ} \mathrm{F}$ above pour point temperature for the blend. The formation of crystals is reversible as heating of the biodiesel can be used to melt the crystals. Alternatively, the crystals can be filtered from the biodiesel.

Stability of the biodiesel is an important attribute if the biodiesel is to be stored for a prolonged period. Poor stability can lead to increasing acid numbers, increasing fuel viscosity, and the formation of gums and sediments. Information about the stability of the stored biodiesel can be achieved by monitoring the acid number and viscosity. Storage stability of biodiesel has not been extensively examined relative to its composition. Therefore, the current best practice involves not storing biodiesel or biodiesel blends for more than six months. The stability of stored diesel fuels can be enhanced by using antioxidants. Examples of acceptable antioxidants include t-butyl hydroquinone (TBHQ), Tenox 21, and tocopherol. Water contamination must be minimized in the stored fuel as it can lead to biological growth in the fuel. If water contamination occurs, biological growth can be mitigated by using biocides.

When selecting tanks for the storage of biodiesel several factors should be considered. Acceptable materials of construction for biodiesel storage tanks include aluminum, steel, Teflon and fluorinated polyethylene or polypropylene. If the tanks to be used have been previously used for the storage of standard diesel fuels, the tanks should be cleaned prior to use for biodiesel storage. Tanks should be selected to minimize the possibility of water contamination. 
Appropriate choice of storage tanks must also be considered for feedstock storage tanks. As with biodiesel storage tanks, the acceptable materials of construction for the feedstock storage tanks are aluminum, steel, Teflon and fluorinated polyethylene or polypropylene. Biodiesel plants based on a feedstock of fats must provide heated storage tanks to keep the feedstock liquid. For vegetable oil feedstocks, the need for heated storage tanks is dictated by the geographical location of the biodiesel plant.

Several factors should be considered when handling biodiesel. While biodiesel contains no hazardous materials, it should still be stored and used in well-ventilated areas. Storage of biodiesel should not be near heat, spark, or flames sources. If stored in drums, proper drum handling procedures should be followed, which includes no drum puncturing, dragging, or sliding. It is recommended that PVC-coated gloves as well as safety glasses or goggles should be used when handling biodiesel.

Typical forms of transportation vessels for biodiesel are rail cars, tank trucks, and drums. Choice of vessel is dictated by the amount of biodiesel being transported. When transporting biodiesel, the transportation vessels should be clean and dry. The preferred transportation vessels would be ones dedicated to biodiesel. While dedicated biodiesel vessels may be possible with drums, it is less likely with tank trucks or rail cars. When using tank truck or rail cars, it is important to specify that the vessel should be steam cleaned and blown dry prior to use if the vessel is not routinely used for biodiesel transport. In addition, the vessels must conform to the same materials of construction used in storage. Seals, gaskets, and adhesives present in the transfer systems should comply with the compatible materials discussed above. Due to its pour point, pure biodiesel is difficult to ship in cold weather. Methods to transport biodiesel in cold weather include hot biodiesel in tank cars that are rapidly delivered, solidified biodiesel in tank cars that are equipped with steam coils, $20 \%$ biodiesel blends with winterized diesel, and $50 \%$ biodiesel blends with kerosene (diesel No. 1), which has a pour point of $0^{\circ} \mathrm{F}$.

Biodiesel is commonly used in blends with standard diesel fuel. As discussed above, use of biodiesel blends can improve the thermal tolerance of the fuel by decreasing the pour point. Therefore, blending may be considered at the biodiesel manufacturing site prior to storage. Several blending techniques can be employed. Biodiesel has a higher specific gravity than diesel fuel $(0.88$ versus 0.85$)$, so blending can be achieved by splashing the biodiesel on the top of diesel. This blending technique is known as splash blending. Alternatively, agitated blending can be used. Agitated blending uses direct mechanical agitation or blending induced by pump the biodiesel/diesel fuel mixture back and forth into a tank truck. While water contamination is undesirable due to the potential to promote biological growth in stored blends, biodiesel/diesel fuel blends will not separate upon exposure to water. 


\section{Product Quality}

Generally, the fuel quality of biodiesel can be influenced by several factors:

- The quality of the feedstock.

- The fatty acid composition of the parent vegetable oil or animal fat.

- The production process and the other materials used in this process.

- Post-production parameters.

The best current measure for biodiesel quality is the ASTM standard, ASTM D 6751: Standard Specification for Biodiesel Fuel (B100) Blend Stock for Distillate Fuels. This standard specifies the properties required for a biodiesel fuel to be used in an engine without problems. Even in blends with conventional diesel fuel, most people in the industry expect that the biodiesel blending stock will meet the standard before being blended. While some properties in the standard, such as cetane number and density, reflect the properties of the chemical compounds that make up biodiesel, other properties provide indications of the quality of the production process. Generally, the parameters given in the standard ASTM D6751 are defined by other ASTM standards. However, other test methods, such as those developed for the American Oil Chemists' Society, (AOCS) may also be suitable (or even more suitable as they were developed for fats and oils and not for petroleum-derived materials addressed in the ASTM standards). This discussion will focus on the most important issues for assuring product quality for biodiesel as it is related to production as well as some post-production parameters.

\section{Production Process Factors}

\section{Completion of reaction}

The most important issue during biodiesel production is the completeness of the transesterification reaction. The basic chemical process that occurs during the reaction is indicated by the following sequence of events:

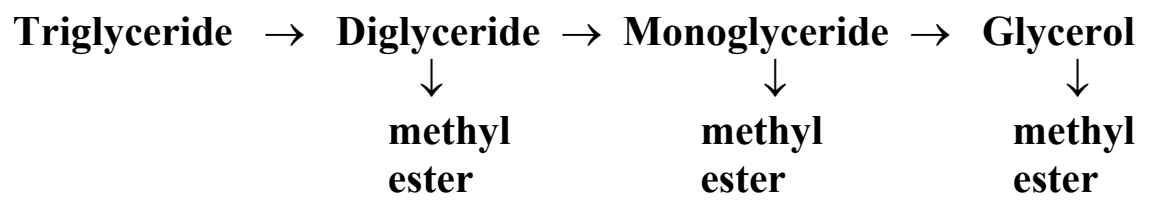

The triglycerides are converted to diglycerides, which in turn are converted to monoglycerides, and then to glycerol. Each step produces a molecule of a methyl ester of a fatty acid. If the reaction is incomplete, then there will be triglycerides, diglycerides, and monoglycerides left in the reaction mixture. Each of these compounds still contains a glycerol molecule that has not been released. These compounds are referred to as bound glycerol. When the bound glycerol is added to the free glycerol, we get the total glycerol. The ASTM specification requires that the total glycerol be less than $0.24 \%$ of the final biodiesel product. The ASTM method requires that the total glycerol be measured using a gas chromatographic method described in ASTM D 6584. Other methods can be used such as a high performance liquid chromatograph (HPLC; for example AOCS Recommended Practice Ca 14b-96: Quantification of Free Glycerine in Selected 
Glycerides and Fatty Acid Methyl Esters by HPLC with Laser Light-Scattering Detection) or a chemical procedure such as that described in AOCS Official Method Ca 14-56 (Total, Free and Combined Glycerol Iodometric Method). All of these techniques require a fairly high level of training and some laboratory equipment.

\section{$\underline{\text { Free Glycerol }}$}

Free glycerol refers to the amount of glycerol that is left in the finished biodiesel. Glycerol is insoluble in biodiesel so almost all of the glycerol is easily removed by settling or centrifugation. Some glycerol may remain either as suspended droplets or a very small amount that is dissolved in the biodiesel, and is known as free glycerol. Most of this glycerol should be removed during the water washing process. Water-washed fuel is generally very low in free glycerol, especially if hot water is used for washing. Distilled biodiesel tends to have a greater problem with free glycerol due to glycerol carry-over during distillation. Fuel with excessive free glycerol will usually have a problem with glycerol settling out in storage tanks, creating a very viscous mixture that can plug fuel filters and cause combustion problems in the engine.

\section{$\underline{\text { Residual Alcohol and Residual Catalyst }}$}

Since methanol (and ethanol) and the alkaline catalysts are more soluble in the polar glycerol phase, most will be removed when the glycerol is separated from the biodiesel. However, the biodiesel may still contain 2-3\% methanol after the separation, which may constitute as much as $40 \%$ of the excess methanol from the reaction. Most plants will recover this methanol by heating the biodiesel. Any methanol remaining after this heating process should be removed by the water washing process. Therefore, the residual alcohol level in the biodiesel should be very low. The allowable alcohol level is specified in European biodiesel standards (give number), but is not included in the ASTM standard. Tests have shown that even as little as $1 \%$ methanol in the biodiesel can lower the flashpoint of the biodiesel from $170 \mathrm{C}$ to less than $40 \mathrm{C}$. Therefore, by including a flashpoint specification of $130 \mathrm{C}$, the ASTM standard limits the amount of alcohol to a very low level $(<0.1 \%)$. Residual alcohol left in the biodiesel will not affect its use in the engine. The amount is too small to negatively impact the fuel's performance. However, lowering the flashpoint presents a potential safety hazard as the fuel may need to be treated more like gasoline, which also has a low flashpoint, than diesel fuel.

Most of the residual catalyst is removed with the glycerol. Like the alcohol, remaining catalyst should be removed during the water washing. Although a value for residual catalyst is not included in the ASTM standard, it will be limited by the specification on sulfated ash. Excessive ash in the fuel can lead to engine deposits and high abrasive wear levels.

\section{Equipment and expertise requirements for quality control of production factors}

All biodiesel production facilities should be equipped with a laboratory so that the quality of the final biodiesel product can be monitored. It is also important to monitor the quality of the feedstocks. 
One strategy used by many producers is to draw a sample of the oil (or alcohol) from each delivery and use that sample to produce biodiesel in the laboratory. This test can be fairly rapid ( 1 or 2 hours) and can indicate whether serious problems are likely in the plant.

Measuring feedstock quality can usually be limited to acid value and water content. These are not too expensive ( $\$ 500$ for the acid value equipment and $\$ 5,000$ for the water measurement equipment) and can be operated by less experienced technicians.

To monitor the completeness of the reaction according to the total glycerol level specified in ASTM D 6751 requires the use of a gas chromatograph and a skilled operator. Large producers will find that having this equipment on-site is necessary. Commercial laboratories (i.e. Williams Laboratory Services) are available that can analyze the samples but the cost is $\$ 100-\$ 150 /$ test and the time required may be several days. Smaller producers will need to use a more robust production process involving extra methanol and probably multiple reaction steps. Then the product quality can be monitored through periodic testing by an outside laboratory.

Other possibilities for monitoring the transesterification reaction and assessing fuel quality are methods based on spectroscopy (such as near-infrared spectroscopy) or physical properties (such as viscometry). These methods, although they are not (yet?) ASTM methods, are usually faster and easier to use than gas chromatography. However, some of them require somewhat extensive calibration. They also cannot accurately quantify glycerol at the low levels called for in the ASTM standard. To circumvent this, comparison to a reaction and product known to meet ASTM standards is needed.

\section{Post-production Factors}

\section{$\underline{\text { Water and Sediment }}$}

Water and sediment contamination are basically housekeeping issues for biodiesel. Water can be present in two forms, either as dissolved water or as suspended water droplets. While biodiesel is generally considered to be insoluble in water, it actually takes up considerably more water than diesel fuel. Biodiesel can contain as much as $1500 \mathrm{ppm}$ of dissolved water while diesel fuel usually only takes up about $50 \mathrm{ppm}$. The standards for diesel fuel (ASTM D 975) and biodiesel (ASTM D 6751) both limit the amount of water to $500 \mathrm{ppm}$. For petroleum-based diesel fuel, this actually allows a small amount of suspended water. However, biodiesel must be kept dry. This is a challenge because many diesel storage tanks have water on the bottom due to condensation. Suspended water is a problem in fuel injection equipment because it contributes to the corrosion of the closely fitting parts in the fuel injection system. Sediment may consist of suspended rust and dirt particles or it may originate from the fuel as insoluble compounds formed during fuel oxidation. Some biodiesel users have noted that switching from petroleumbased diesel fuel to biodiesel may cause an increase in sediment that comes from deposits on the walls of fuel tanks that had previously contained diesel fuel. Because its solvent properties are different from diesel fuel, biodiesel may loosen sediments and cause fuel filter plugging during the transition period. 


\section{$\underline{\text { Storage Stability }}$}

Storage stability refers to the ability of the fuel to resist chemical changes during long term storage. These changes usually consist of oxidation due to contact with oxygen from the air. The fatty acid composition of the biodiesel fuel is an important factor in determining stability towards air. Generally, the more unsaturated fatty acids (C18:2, linoleic acid; C18:3 linolenic acid) are more susceptible towards oxidation. The changes can be catalyzed by the presence of certain metals (including those making up the storage container) and light. If water is present, hydrolysis can also occur. The chemical changes in the fuel associated with oxidation usually produce hydroperoxides which can, in turn, produce short chain fatty acids and aldehydes. Under the right conditions, the hydroperoxides can also polymerize. Therefore, oxidation is usually denoted by an increase in the acid value and viscosity of the fuel. Often these changes are accompanied by a darkening of the biodiesel color from yellow to brown and the development of a "paint" smell.

When water is present, the esters can hydrolyze to long chain free fatty acids which also cause the acid value to increase.

There is currently no generally accepted method for measuring the stability of biodiesel. The techniques generally used for petroleum-based fuels, such as ASTM D 2274, have been shown to be incompatible with biodiesel. Other procedures, such as the Oil Stability Index or the Rancimat apparatus, which are widely used in the fats and oils industry, seem to be more appropriate for use with biodiesel. However, the engine industry has no experience with these tests and acceptable values are not known. Also, the validity of accelerated testing methods has not been established or correlated to actual engine problems. If biodiesel's acid number, viscosity, or sediment content increase to the point where they exceed biodiesel's ASTM limits, the fuel should not be used as a transportation fuel.

Additives such as BHT and TBHQ (t-butylhydroquinone) are common in the food industry and have been found to enhance the storage stability of biodiesel. Biodiesel produced from soybean oil naturally will contain some antioxidants (tocopherols, i.e., vitamin E), providing some protection against oxidation (some tocopherol is lost during refining of the oil prior to biodiesel production). Any fuel that will be stored for more than 6 months, whether it is diesel fuel or biodiesel, should be treated with an antioxidant additive. 


\section{Laboratory - Exercise 3}

\section{Design of a Process for an Arbitrary Feedstock}

Divide the class into four groups. Have each group develop a process flow diagram for a plant that would use one of the following feedstocks and produce fuel-grade biodiesel.

1. Crude soybean oil

2. Refined soybean oil

3. Yellow grease

4. Untreated restaurant waste

5. Trap grease

After the groups have completed their discussions, have them present and defend their designs to the entire class. 


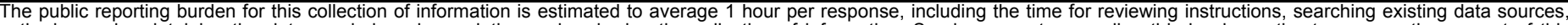

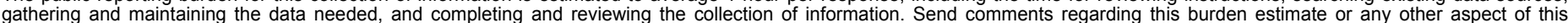

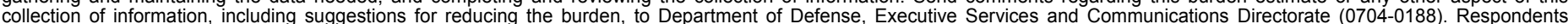

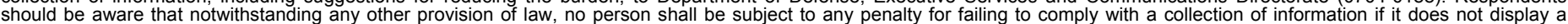

should be aware that notwithstanding

PLEASE DO NOT RETURN YOUR FORM TO THE ABOVE ORGANIZATION.
1. REPORT DATE (DD-MM-YYYY)
July 2004

\section{REPORT TYPE \\ Subcontract}

4. TITLE AND SUBTITLE

Biodiesel Production Technology
3. DATES COVERED (From - To) August 2002 - January 2004

5a. CONTRACT NUMBER

DE-AC36-99-G010337

5b. GRANT NUMBER

5c. PROGRAM ELEMENT NUMBER

5d. PROJECT NUMBER

NREL/SR-510-36244

5e. TASK NUMBER

BB044630

5f. WORK UNIT NUMBER
7. PERFORMING ORGANIZATION NAME(S) AND ADDRESS(ES)

lowa State University, 2207 Pearsen Hall, Ames IA

Renewable Products Development Laboratory

USDA/NCAUR

9. SPONSORING/MONITORING AGENCY NAME(S) AND ADDRESS(ES)

National Renewable Energy Laboratory

1617 Cole Blvd.

Golden, CO 80401-3393 8. PERFORMING ORGANIZATION
REPORT NUMBER

ACO-2-31056-01
10. SPONSOR/MONITOR'S ACRONYM(S) NREL

11. SPONSORING/MONITORING AGENCY REPORT NUMBER NREL/SR-510-36244

12. DISTRIBUTION AVAILABILITY STATEMENT

National Technical Information Service

U.S. Department of Commerce

5285 Port Royal Road

Springfield, VA 22161

13. SUPPLEMENTARY NOTES

NREL Technical Monitor: K. Shaine Tyson

14. ABSTRACT (Maximum 200 Words)

Biodiesel is an alternative fuel for diesel engines that is gainign attention in the United States after reaching a considerable level of success in Europe. The purpose of this book is to describe and explain the process and issues involved in producing this fuel.

\section{SUBJECT TERMS}

biodiesel; production; transesterfication

\begin{tabular}{l}
\hline 16. SECURITY CLASSIFICATION OF: \\
\begin{tabular}{l|l|l|}
\hline a. REPORT & b. ABSTRACT & c. THIS PAGE \\
Unclassified & Unclassified & Unclassified \\
& & \\
\end{tabular} \\
\hline
\end{tabular}

\begin{tabular}{|c|c|}
\hline $\begin{array}{l}\text { 17. LIMITATION } \\
\text { OF ABSTRACT }\end{array}$ & $\begin{array}{l}\text { 18. NUMBER } \\
\text { OF PAGES }\end{array}$ \\
\hline UL & \\
\hline
\end{tabular}

19a. NAME OF RESPONSIBLE PERSON

19b. TELEPONE NUMBER (Include area code) 